\title{
Reconfigurable Millimeter-Wave Components Based on Liquid Crystal Technology for Smart Applications
}

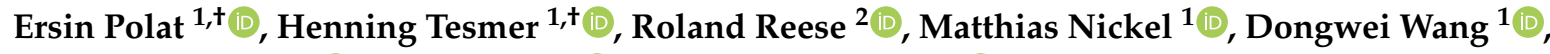 \\ Peter Schumacher ${ }^{1}{ }^{\mathbb{D}}$, Rolf Jakoby ${ }^{1}\left(\mathbb{D}\right.$ and Holger Maune ${ }^{1, *(\mathbb{B}}$ \\ 1 Institute of Microwave Engineering and Photonics, Technische Universität Darmstadt, 64283 Darmstadt, \\ Germany; polat@imp.tu-darmstadt.de (E.P.); tesmer@imp.tu-darmstadt.de (H.T.); \\ nickel@imp.tu-darmstadt.de (M.N.); wang@imp.tu-darmstadt.de (D.W.); \\ schumacher@imp.tu-darmstadt.de (P.S.); jakoby@imp.tu-darmstadt.de (R.J.) \\ 2 Deutsche Telekom Technik GmbH, 64295 Darmstadt, Germany; Roland.Reese@telekom.de \\ * Correspondence: maune@imp.tu-darmstadt.de; Tel.: +49-6151-16-28450 \\ + These authors contributed equally to this work.
}

Received: 30 March 2020; Accepted: 23 April 2020; Published: 27 April 2020

check for updates

\begin{abstract}
This paper presents recent development of tunable microwave liquid crystal (LC) components in the lower millimeter wave $(\mathrm{mmW})$ regime up to the $\mathrm{W}$-band. With the utilization of increasing frequency, conventional metallic waveguide structures prove to be impractical for LC-based components. In particular, the integration of the electric bias network is extremely challenging. Therefore, dielectric waveguides are a promising alternative to conventional waveguides, since electrodes can be easily integrated in the open structure of dielectric waveguides. The numerous subcategories of dielectric waveguides offer a high degree of freedom in designing smart millimeter wave components such as tunable phase shifters, filters and steerable antennas. Recent research resulted in many different realizations, which are analyzed in this paper. The first demonstrators of phased array antennas with integrated LC-based phase shifters are reviewed and compared. In addition, beam steering with a single antenna type is shown. Furthermore, the possibility to realize tunable filters using LC-filled dielectric waveguides is demonstrated.
\end{abstract}

Keywords: liquid crystal; millimeter wave devices; steerable antennas; dielectric waveguide; tunable filters

\section{Introduction}

The ever-rising demand of higher data rates is an ongoing trend in today's society. It is predicted that the data traffic will be 10,000 times higher in 2025 than it was back in 2013 [1]. The imminent 5th generation standard (5G) [2] is meant to satisfy this demand. With no sign of stagnation in data rates, a subsequent 6th generation standard has to utilize frequencies in the millimeter-wave $(\mathrm{mmW})$ regime also for communications. The necessary large bandwidths can be provided at these frequencies, since a fixed relative bandwidth translates to a higher absolute bandwidth when increasing the operation frequency. Possible usable spectra for high data rate communications have been investigated at frequencies in V-band (50 GHz to $75 \mathrm{GHz}$ ) and $\mathrm{W}$-band $(75 \mathrm{GHz}$ to $100 \mathrm{GHz})[3,4]$. Besides other challenges in communications at these frequencies, the main obstacles to overcome for wireless communication are the significant free space loss, which increases with rising frequency, and atmospheric attenuation effects, which are frequency-dependent. Figure 1 depicts the attenuation versus transmission range for $12 \mathrm{GHz}, 60 \mathrm{GHz}$ and $100 \mathrm{GHz}$. Uncontrollable effects, such as change in weather, can cause higher attenuation, hence the usable range for reliable communication is even less than Figure 1 might imply. 


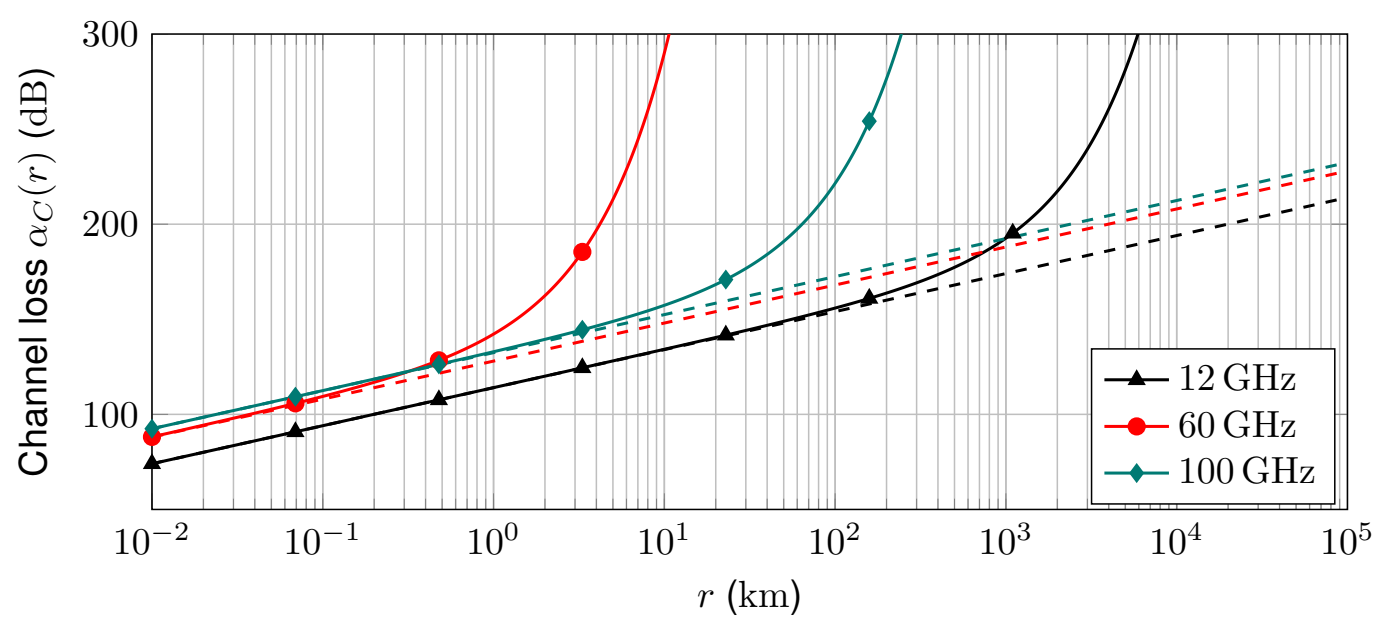

Figure 1. Channel attenuation versus transmission range. Dashed lines represent free-space loss, while solid lines represent the combination of free-space loss together with atmospheric attenuation effects.

In order to compensate for the high signal attenuation, either the transmission power or the gain of the used antennas has to be increased. Since output power is a limited quantity, especially in mobile applications, it is more convenient to employ antennas with high gain. High gain, however, is linked to a small half-power beam width (HPBW) as

$$
g=\eta \cdot D \approx \eta \cdot \frac{4 \pi}{\mathrm{HPBW}_{\mathrm{az}} \cdot \mathrm{HPBW}_{\mathrm{el}}}
$$

states, where $\eta$ is the antenna efficiency and $D$ is the directivity of the antenna, which can be approximated by the HPBW of the radiation pattern in azimuth and elevation plane. With a small beamwidth, precise alignment of transmitter and receiver antennas is mandatory in order to ensure an operational system. Hence, the antennas' beams have to be steerable in order to correct small misalignment and to enable communication in non-stationary scenarios. The straight forward approach for steering an antenna's beam is by mechanically turning the whole antenna to the desired angular position. However, mechanical steering requires motors, which consume space, add additional weight and power consumption to the system and have need of maintenance. Therefore, an electronically controlled beam steering approach, where the antenna remains static in its position, is favorable.

The most common approach for electrically steering the antenna beam is the phased array concept. A phased array consists of a group of antenna elements which acts as one transmitting/receiving entity. However, each individual antenna can be addressed such that it radiates the transmitted signal with a controllable delay in time with respect to its neighbors. As a result, the radiated signal can be steered to different directions. Figure 2 depicts a simplified representation of a phased array system. Considering the transmitting case, the signal intended to be transmitted has to be divided to the different branches leading to each individual antenna. In order to achieve different time delay in the branches to each antenna element, tunable phase shifters are employed. As a result, the antenna elements radiate the signal with different phase, and a steered direction of radiation can be achieved.

An additional challenge is the continuously increasing number of services and network participants, which makes the available spectrum a scarce and valuable resource. Therefore, it must be used as efficient as possible by applying dynamic spectrum management systems such as bandless or seamless cognitive radio systems. For these systems, reconfigurable radio frequency (RF) front ends are key components. State-of-the-art front ends use filter banks to switch between different discrete filters, which have a fixed filter characteristic. Therefore, only predefined channels can be selected and no adaptation is possible. In order to increase the spectrum efficiency, the filter needs to be reconfigurable, which in turn makes the whole system reconfigurable. With such a filter included, the system is not limited to predefined channels or services. Furthermore, tunable filters are compact solutions when 
compared to filter banks. Tunablity of the resonance frequency can be realized by varying the electrical size of the filter's resonators. Hence, either the physical dimensions of the resonators or the material's permittivity have to be changed.

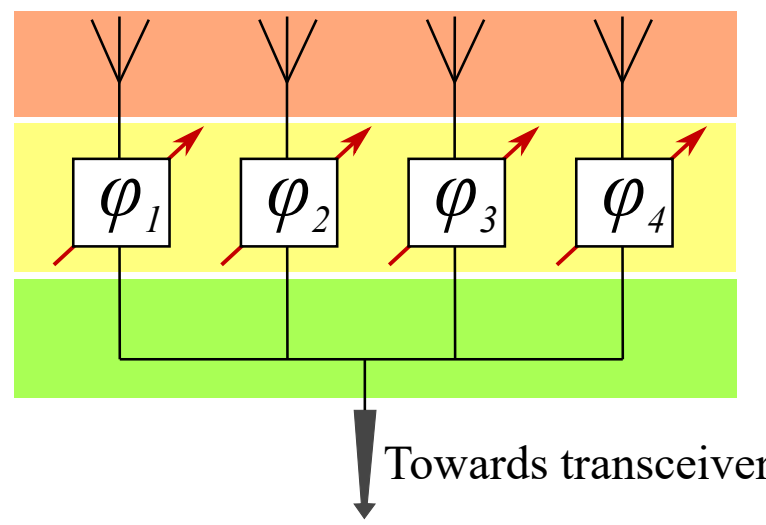

\section{Antenna elements}

Phase shifters

Power divider/combiner

Figure 2. Schematic representation of an antenna array using analog beam steering.

There are different tuning mechanisms, which can be used for phase shifters, antenna arrays, or filters. In this work, tunable liquid crystal (LC) based components are considered. LC is an anisotropic material which shows a mesophase between crystal and liquid. In this special material state, order of molecule orientation as in a crystal is present, but the molecules are able to rotate and flow as in a liquid. By controlling the orientation of the molecules with respect to the applied RF field, the macroscopic relative permittivity $\varepsilon_{r}$ and loss tangent $\tan \delta$ can be varied. The molecule orientation can be influenced by the use of external (quasi-)static electric or magnetic bias fields. The used nematic LC molecules tend to align themselves in parallel to the corresponding field lines. While magnetic fields are mainly used for proof-of-concept and reference purposes, electric bias is commonly preferred, since it offers versatility in field strength and orientation by simply changing the potential distribution at the employed electrode network. While maintaining the RF-polarization, the external field can change the molecule alignment and lead to continuously tunable permittivity between the extreme cases of parallel and orthogonal RF polarization $\varepsilon_{r, \|}$ and $\varepsilon_{r, \perp}$, respectively, with respect to the long molecule axis. For the first LC based microwave components, standard display LC mixtures have been utilized, such as K15 (5CB) or E7, as in [5,6]. As depicted in Figure 3, they have comparatively high loss and small anisotropy.

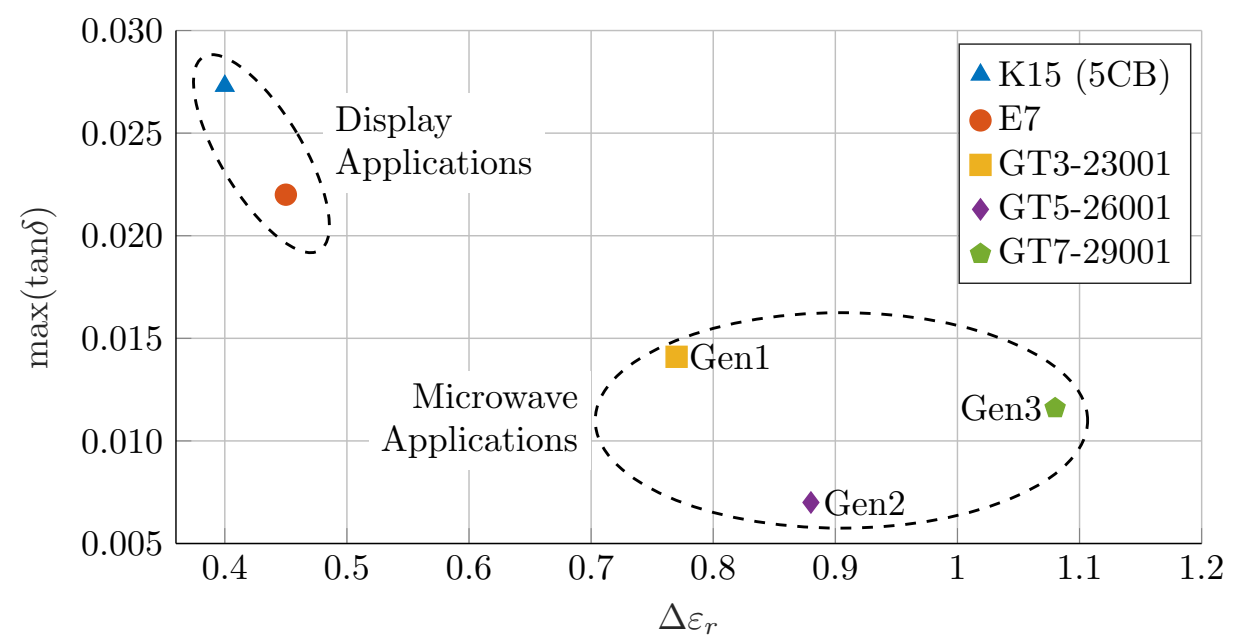

Figure 3. Dielectric anisotropy versus maximum loss tangent of different LC mixtures at $19 \mathrm{GHz}$. Values provided by Merck KGaA and [7]. Gen1 to Gen3 indicate the different generations of the GT-series. 
Therefore, special microwave LC mixtures have been developed over the past two decades, which led to various microwave LC mixtures, e.g., GT3-23001, GT5-26001 and GT7-29001 from Merck $\mathrm{KGaA}$, and development is still ongoing [8]. Figure 3 compares realizable dielectric anisotropy and maximum dissipation factor of five different LC mixtures. The frequency of $19 \mathrm{GHz}$ is chosen since data for all five LC mixtures is present at this frequency. For microwave applications combination of low maximum loss tangent and high dielectric anisotropy is desired, hence the closer a LC mixture is to the bottom right corner of the graph, the more favorable it is. Therefore, the work throughout the rest of this paper focuses on devices which utilize LC mixtures from the GT-series, which are summarized in Table 1. Another important specification is the response time of the LC mixture, which depends on the viscosity $\gamma_{r}$ and anisotropy at the frequency of the bias field (usually around $\left.1 \mathrm{kHz}\right) \Delta \varepsilon_{1 \mathrm{kHz}}$. A higher $\Delta \varepsilon_{1 \mathrm{kHz}}$ reduces the response time, while a higher $\gamma_{r}$ increases the response time. In terms of loss the GT5-26001 has the lowest maximum dissipation factor, whereas its response time is the slowest, see Table 1. The latest LC mixture GT7-29001 has the largest anisotopy, the fastest response time and still low loss. Furthermore, in $[9,10]$ it has been shown for the GT3-23001 mixture that the material properties are almost constant in the $\mathrm{mmW}$ regime up to several THz.

Table 1. Different LC mixtures used in this manuscript and their corresponding properties. Data provided by Merck KGaA at $19 \mathrm{GHz}$.

\begin{tabular}{lcccccc}
\hline LC Mixture & $\varepsilon_{r, \|}$ & $\varepsilon_{r, \perp}$ & $\tan \delta_{\|}$ & $\tan \delta_{\|}$ & $\gamma_{r}(\mathbf{m P a ~ s})$ & $\Delta \varepsilon_{\mathbf{1 ~ k H z}}$ \\
\hline GT3-23001 & 3.28 & 2.46 & 0.0038 & 0.0143 & 727 & 4.6 \\
GT5-26001 & 3.27 & 2.39 & 0.0022 & 0.007 & 1958 & 1.0 \\
GT7-29001 & 3.53 & 2.46 & 0.0064 & 0.0116 & 307 & 22.1 \\
\hline
\end{tabular}

Besides the development of LC mixtures, the corresponding millimeter-wave components have undergone a change of topologies. Therefore, this paper starts where [11] concluded and highlights important developments and advances in reconfigurable, LC-based millimeter-wave components up to the W-band. Different approaches offer a varying spectrum of performance and complexity regarding bias network and assembly. Advantages from moving from metallic waveguide to dielectric waveguide topologies are highlighted throughout the manuscript. The different color-coded sections in Figure 2 are addressed in the paper.

Focus is put on different dielectric topologies for tunable LC phase shifters in the first section. The second section covers demonstrators for phased arrays with dielectric phase shifters and antennas. Furthermore, the power divider network of a dielectric antenna array is addressed. Moreover, a different beam steering approach besides the phased array is reviewed. Afterwards, a tunable filter using a dielectric waveguide for band selection is presented, before conclusions are drawn at the end of the paper.

\section{Reconfigurable Components Based on Liquid Crystal}

\subsection{Phase Shifters}

In this section, a summary of different tunable phase shifters based on LC technology for the lower $\mathrm{mmW}$ regime is presented. Besides phased array antenna applications [12], tunable phase shifters are a key component for several RF system, e.g., phase modulators [13] or wireless local area networks (WLAN) using multiple-input multiple-output (MIMO) technology [14]. The reviewed phase shifters are all passive delay lines, where phase shift is obtained by a delay of the guided signal. However, the achieved phase shift is frequency dependent, due to the frequency dependency of the wave period. The phase shifters vary the electrical length instead of the physical length in order to obtain a phase shift. This is realized by utilizing the anisotropic permittivity of the LC molecules, which can be controlled by orienting the direction of the LC molecules with respect to the applied 
RF field. Since LC molecules can be continuously oriented, the phase shifters are also continuously tunable. The maximum differential phase shift of an LC phase shifter is given by [15]

$$
\Delta \varphi=l_{P S} \cdot\left(\Delta \beta \cdot \frac{180^{\circ}}{\pi}\right)=l_{P S} \cdot\left(\left(\sqrt{\varepsilon_{r, \|}}-\sqrt{\varepsilon_{r, \perp}}\right) \cdot \frac{360^{\circ} \cdot f}{c_{0}}\right),
$$

with the length $l_{P S}$, differential phase constant $\Delta \beta=\beta_{\|}-\beta_{\perp}$, where $\beta_{\|}$and $\beta_{\perp}$ are the phase constant for parallel and orthogonal LC orientation, respectively. For comparison of these phase shifters, the following two parameters are considered. First, the steering efficiency

$$
\tau_{\varphi}=\frac{\Delta \varphi}{l_{P S}}
$$

which defines the differential phase shift per unit length, which is considered if a compact phase shifter is needed. Second, the Figure-of-Merit (FoM) which is defined by

$$
\mathrm{FoM}=\frac{\Delta \varphi_{\max }}{\mathrm{IL}_{\max }}
$$

where $\mathrm{IL}_{\max }$ is the maximum insertion loss (IL) for all tuning states. In contrast to the steering efficiency, the FoM is a performance parameter without considering the phase shifter's dimensions.

As mentioned above, LC can be tuned either by an external static magnetic or a quasi-static electric field. For magnetic biasing either permanent magnets are mechanically turned around the phase shifter or electromagnets can be used. However, both magnetic biasing concept are impractical for most applications, since the whole setup becomes bulky, and furthermore, exhibit a huge power consumption either to move the magnets or to generate the necessary magnetic field strengths. Therefore, electric biasing is preferred for the most applications, since the electrodes can be integrated inside the phase shifters. Continuous tuning can be obtained by varying the potential distribution. Such an electrical biasing system for a hollow waveguide phase shifter is shown in Figure 4. It enables the necessary bias field configurations for parallel and orthogonal LC orientation. The electrodes are processed on a thin substrate to provide an isolation between waveguide and electrode. However, parasitic modes can be excited in this substrate, which degenerate the RF performance. Therefore, stepped-impedance lines are usually used as electrodes to prevent the excitation of these parasitic modes [16,17].

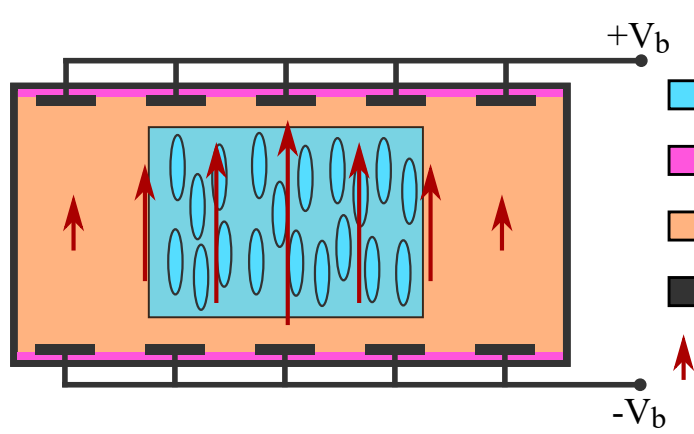

(a)

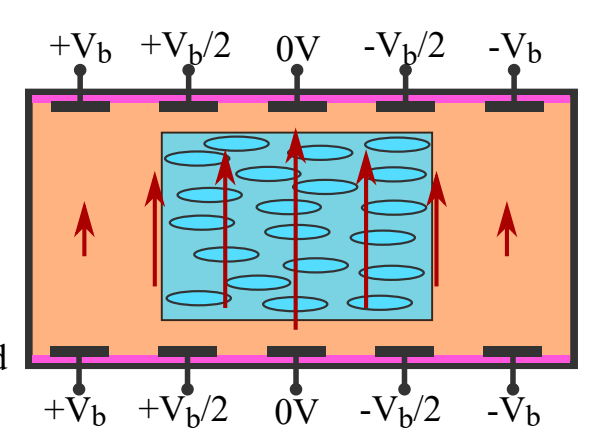

(b)

Figure 4. Cross section of an LC waveguide phase shifter, as presented in [16,17]. The waveguide is filled with a dielectric which is utilized as an LC cavity. For electric LC orientation, an electrode system is integrated on a thin substrate inside the waveguide. $(\mathbf{a}, \mathbf{b})$ show the bias configuration for parallel and orthogonal LC biasing, respectively.

In literature, different waveguide topologies have been used to realize LC phase shifters. Depending on the application and desired frequency range, each of them has its advantages and disadvantages. For $K_{a}$-band applications, hollow waveguide LC phase shifters $[16,17]$ have the best 
performance in terms of FoM and losses. In [17] a maximum FoM of around $150 \%$ dB with electric biasing has been demonstrated, which is to the authors' best knowledge the highest FoM of an LC phase shifter with electric biasing. However, for higher frequencies, the design and realization of such waveguide phase shifters becomes very challenging, due to the decreasing dimensions. Therefore, LC waveguide phase shifters with integrated electrode systems have only been demonstrated successfully up to the $\mathrm{K}_{\mathrm{a}}$-band. W-band LC phase shifters [18] achieve a high FoM of around $135^{\circ} / \mathrm{dB}$ but only with magnetic biasing. An electrode system for a W-band LC phase shifter is presented in [19], but the design was only verified in simulations.

Hence, other waveguide topologies are considered for LC phase shifters at higher frequencies. Dielectric waveguides are very promising candidates, because their open structure allows an easy electrode integration, and furthermore, the intrinsic dielectric can be used to form a cavity for LC. There are different topologies of dielectric waveguides, which can be used for LC phase shifter designs. A selection of the most important ones is shown in Figure 5. Figure 5a shows a subwavelength (sWL) fibre, which has the simplest structure. As the name indicates, its cross-section is smaller than the guided wavelength and is around $\lambda_{0} / 2$. The wave is guided inside the fibre and in air. This enables a comparatively low-loss wave guidance compared with dielectric waveguides where the wave is fully guided in the dielectric. However, for LC applications, the evanescent field in air can be influenced by the biasing electrodes. Therefore, they need to be placed with a certain distance to the sWL such that they do not disturb the guided wave. As a consequence, higher bias voltages are needed. The most known dielectric waveguide is the stepped-index fibre (SIF), as depicted in Figure $5 \mathrm{~b}$. It consists of a dielectric core and cladding with different permittivities $\varepsilon_{1}>\varepsilon_{2}>\varepsilon_{0}$. The wave is fully guided in the fibre, since the evanescent fields are decaying inside the cladding. Therefore, electrodes can directly be placed on the cladding without influencing the propagating wave. However, the cladding's material permittivity must be smaller than the LC mixture. Therefore, the selection of suitable materials is limited.

In contrast to the previous dielectric waveguides, the following topologies consist of at least one metal plate, which acts also as a mechanical support. The dielectric image line (DIL), see Figure $5 \mathrm{c}$, is obtained by placing a metallic image plane below a halved sWL fibre. A main advantage for the DIL for LC applications is the reduced height of the dielectric material. Since the LC switching time depends on the height of the LC cavity [15], faster switching times are obtained or lower bias voltages are needed. The parallel plate dielectric waveguide (PPDW) and nonradiative dielectric waveguide (NRD), depicted in Figure 5d,e respectively, have a similar structure, but the dimensions and polarization are different. Both have a dielectric core which is placed between two parallel metal plates. For the PPDW the fundamental $\mathrm{TE}_{10}$ mode is polarized orthogonal to the plates, whereas for the NRD the operating higher order mode $\mathrm{LSM}_{01}$ is polarized parallel to the plates. As the name indicates, the NRD is nonradiative even at bends and discontinuities, which is unique characteristic compared to other dielectric waveguides. By having a plate separation of $b<\lambda_{0} / 2$, propagation is only possible in and along the dielectric slab. Therefore, in contrast to other dielectric waveguide topologies, the NRD is suitable for a filter design, since it does not suffer from radiation losses. The following subsections highlight the different dielectric waveguide topologies in detail. 


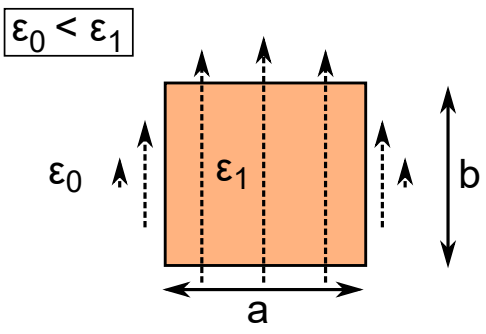

(a) Subwavelength fibre (sWL)

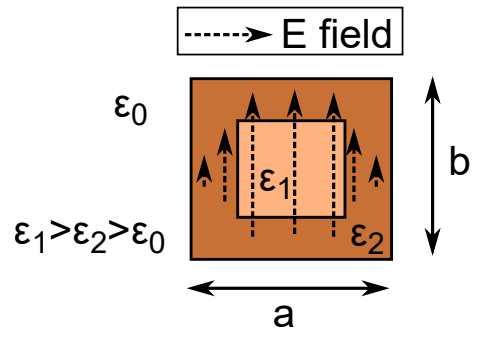

(b) Stepped-index fibre (SIF)

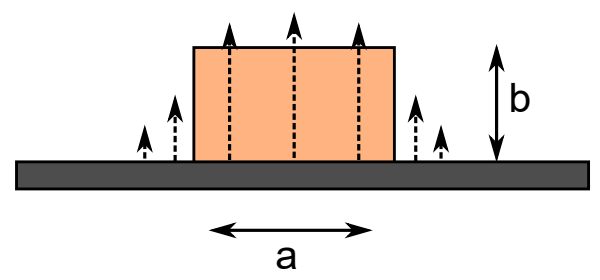

(c) Dielectric image line (DIL)

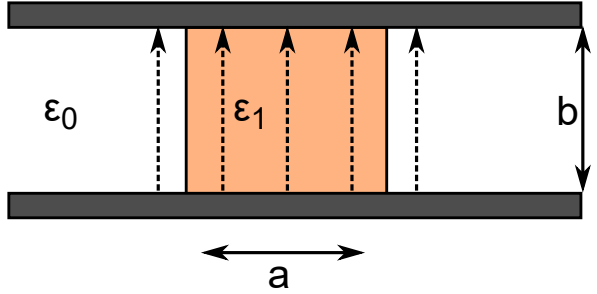

(d) Parallel plate dielectric waveguide (PPDW)

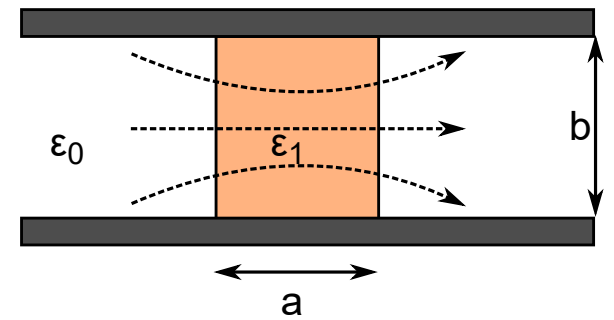

(e) Nonradiative dielectric waveguide (NRD)

Figure 5. Overview of dielectric waveguide topologies, which have been used for LC phase shifters. (a) The rectangular sWL fiber guides the wave in the dielectric and air. (b) The SIF consists of a core and cladding material. The wave is completely guided in the dielectrics. (c) For the DIL a subwavelength waveguide is halved an mounted on a metal plane. (d) The PPDW consisits of a dielectric slab which is inserted between to parallel metal plates. (e) The NRD looks similar to the PPDW, except that the dimensions and polarization differ.

\subsubsection{Subwavelength Fibre}

The design of a sWL LC phase shifter is comparatively very simple. Since LC is liquid, it must be placed inside a cavity. Therefore, for the sWL fibre, a dielectric with a dielectric constant similar to the used LC is necessary. In [20], Rexolite is used, as its relative permittivity of $\varepsilon_{r}=2.53$ [21] fits well with the LC mixture. Furthermore, it is well processable by standard machining. A schematic of the cross section of the sWL phase shifter is shown in Figure 6. The sWL fibre has a quadratic cross section of $1.8 \mathrm{~mm} \times 1.8 \mathrm{~mm}$ and the LC section has a length of $33 \mathrm{~mm}$. the LC section has a rectangular shape for polarization stabilization, as depicted in Figure 6. The sWL phase shifter is manufactured by milling two Rexolite halves, which are glued together with ultraviolet (UV) adhesive afterwards. The fabricated LC phase shifter filles with GT7-29001 is shown in Figure 7. Due to the evanescent fields in air, the electrodes must be placed at certain distance to not disturb these fields, as shown in Figure $7 \mathrm{~b}$. Simple copper plates are used as electrodes. Bias is applied with a rectangular $1 \mathrm{kHz}-\mathrm{AC}$ voltage of $V_{p p}=500 \mathrm{~V}$. To measure the phase shifter, transitions from WR-10 hollow waveguide to sWL fibre are designed. These are described in detail in [20]. The S-parameter results are shown in Figure 8. 


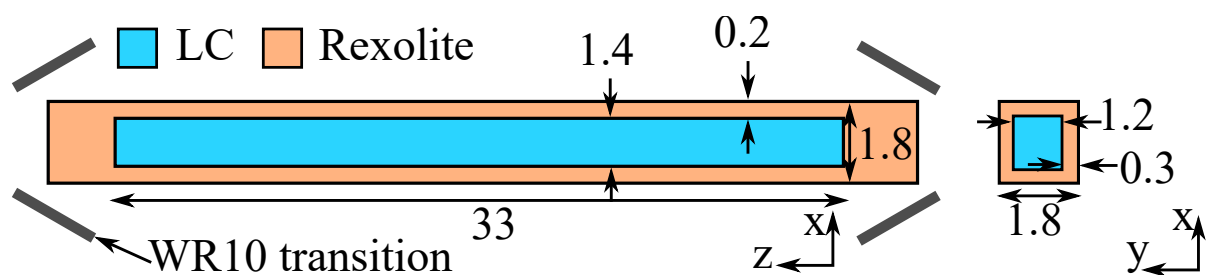

Figure 6. Cross section of an LC sWL phase shifter for W-band [20]. All dimensions are given in mm.

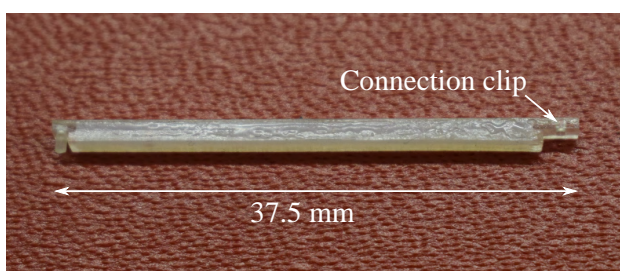

(a)

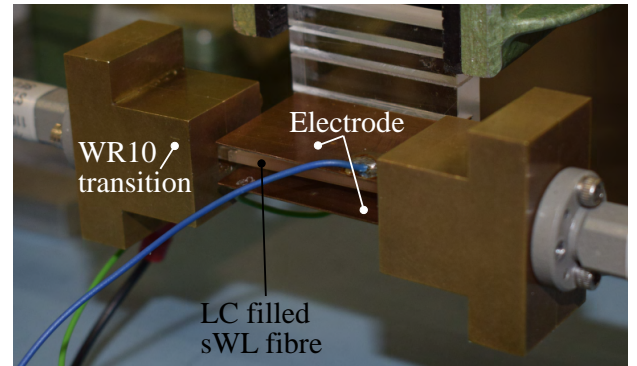

(b)

Figure 7. (a) Fabricated LC sWL fibre, as presented in [20]. (b) Connection clips are designed to hold the phase shifter in place for measurement. Two copper plates are used as electrodes for LC biasing.

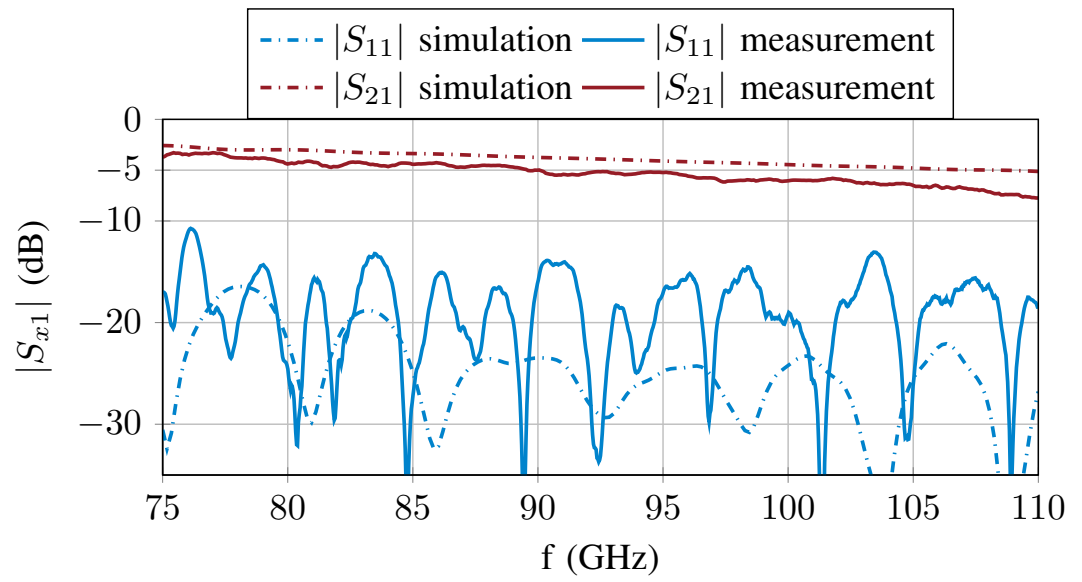

Figure 8. Measured and simulated S-parameters of the tunable sWL fibre phase shifter.

A difference of around $1 \mathrm{~dB}$ in the transmission coefficient $S_{21}$ exists between measurement and simulation results. A reason for this is the glue, which is used to seal the LC cavity. Since, the losses of the glue are not known, they cannot be taken into account in simulations. Furthermore, the reflection coefficient $S_{11}$ is higher as in the simulation. A reason for this is the modular assembly of the phase shifter, as shown in Figure 7a, where connection clips are used on both sides to connect the phase shifter to the measurements setup, shown in Figure 7b. In Figure 9, the simulated and measured differential phase shift and FoM is depicted. The measured differential phase shift is ranging from $400^{\circ}$ to $900^{\circ}$, which is around $120^{\circ}$ less as simulated. This is caused by non-perfect alignment of the LC molecules, and furthermore, the material parameters of the LC mixture are only available at $19 \mathrm{GHz}$. It has been shown that the anisotropy is slightly decreasing in the millimetre-wave regime [9], which is decreasing the maximum differential phase shift. Due to the lower phase shift and higher losses, the measured FoM is lower as simulated. Nevertheless, an average FoM of $130 \% \mathrm{~dB}$ from $85 \mathrm{GHz}$ to $110 \mathrm{GHz}$ was obtained with electrical biasing. Furthermore, a maximum steering efficiency $\tau_{\varphi}=27.3^{\circ} / \mathrm{mm}$ was achieved. 


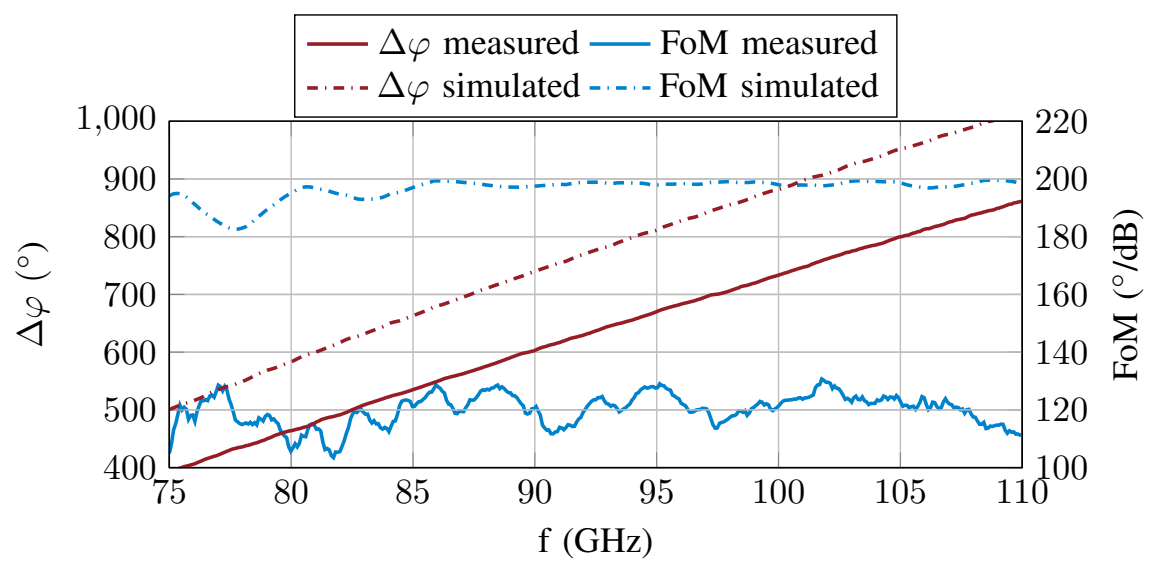

Figure 9. Measured and simulated FoM and differential phase shift of the sWL phase shifter.

\subsubsection{Stepped-Index Fibre}

For the SIF phase shifter, LC is used as core material. To obtain total reflection between $\mathrm{LC}$, the permittivity of the cladding must be lower as for LC, hence $\varepsilon_{r, \text { cladding }}<\varepsilon_{r, \perp}$. However, most LC mixtures have a $\varepsilon_{r, \perp} \approx 2.4$ which limits the number of suitable cladding materials, especially considering the LC integration and sealing. Nevertheless, Rexolite is used as cladding material with a $\varepsilon_{r}=2.53$ in [22]. This is slightly higher than the orthogonal permittivity of the used LC mixture GT5-26001, see Table 1. Hence, the design has to be adapted to guarantee propagation for all LC orientations. The resulting design for the $\mathrm{W}$-band is shown in Figure 10. It consists of an LC core with a length of $l_{\mathrm{LC}}=19 \mathrm{~mm}$ and a cross section of $h_{\mathrm{LC}}=2.2 \mathrm{~mm}$ and $b_{\mathrm{LC}}=1.4 \mathrm{~mm}$. The cladding width is reduced on all sides, to reduce the effective permittivity and obtain propagation. For better matching, a taper made out of Rogers 3003 is inserted at the ends of the LC cavity. The manufactured SIF phase shifter with two WR-10 transitions for the measurements is shown in Figure 11. For biasing, adhesive copper bands are directly glued on the cladding. Since the propagating wave is decaying inside the cladding, no additional distance is needed as for the sWL fibre phase shifter. The simulated and measured S-parameter results are shown in Figure 12. A rectangular $1 \mathrm{kHz}-\mathrm{AC}$ bias voltage of $V_{p p}=1100 \mathrm{~V}$ was applied.

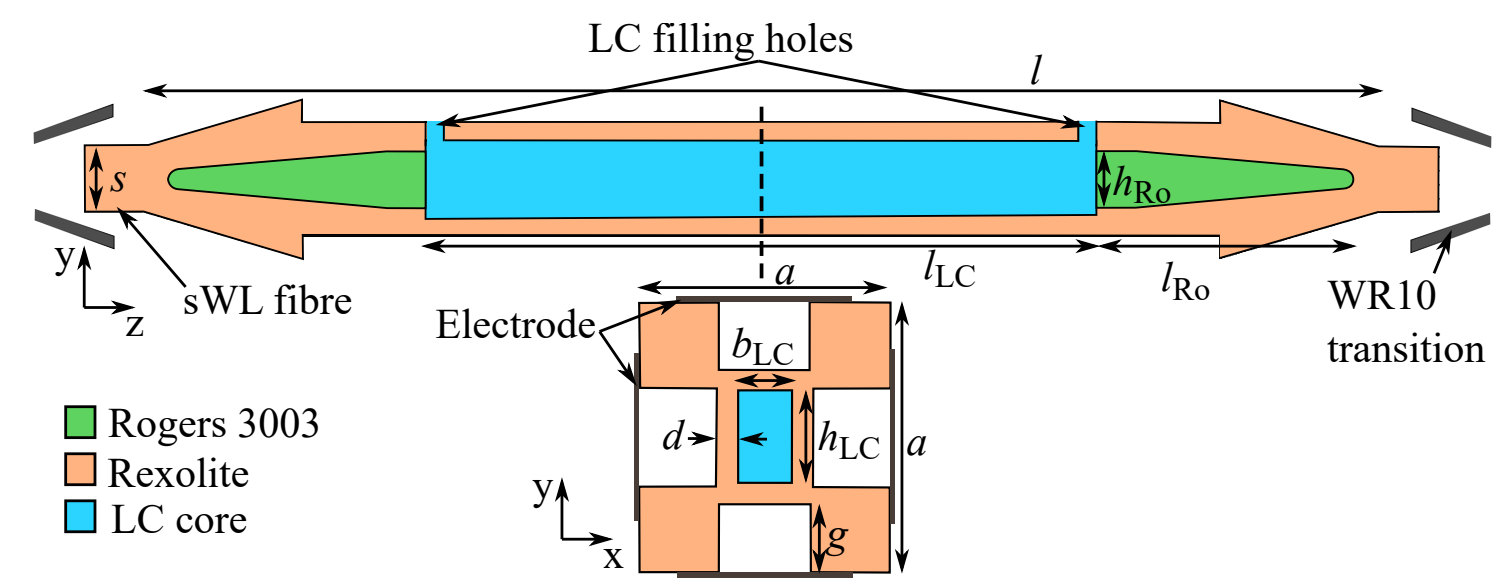

Figure 10. Schematic of the SIF LC phase shifter, presented in [22]. All dimensions in mm: $l=35$, $l_{L C}=19, l_{R o}=6.28, h_{R o}=1.6, h_{L C}=2.2, b_{L C}=1.4, a=6.5, d=0.55, g=2, s=1.8$. 


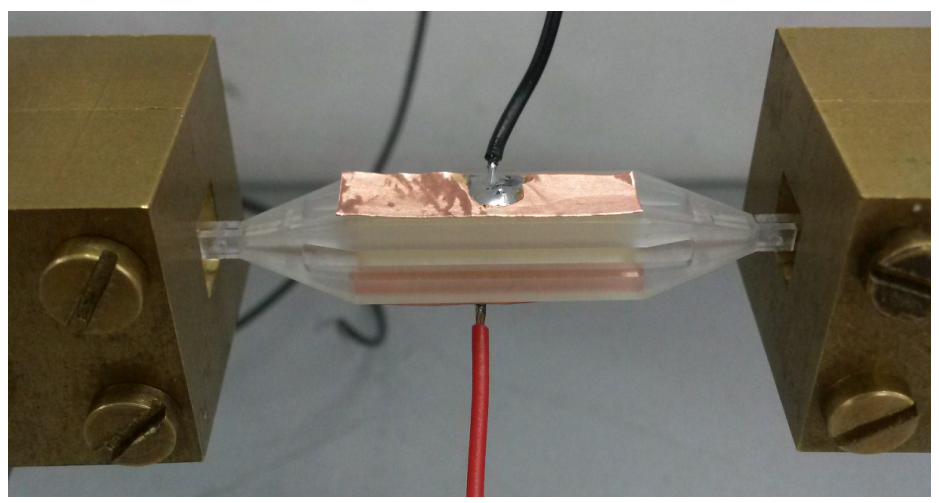

Figure 11. Fabricated SIF LC phase shifter presented in [22].

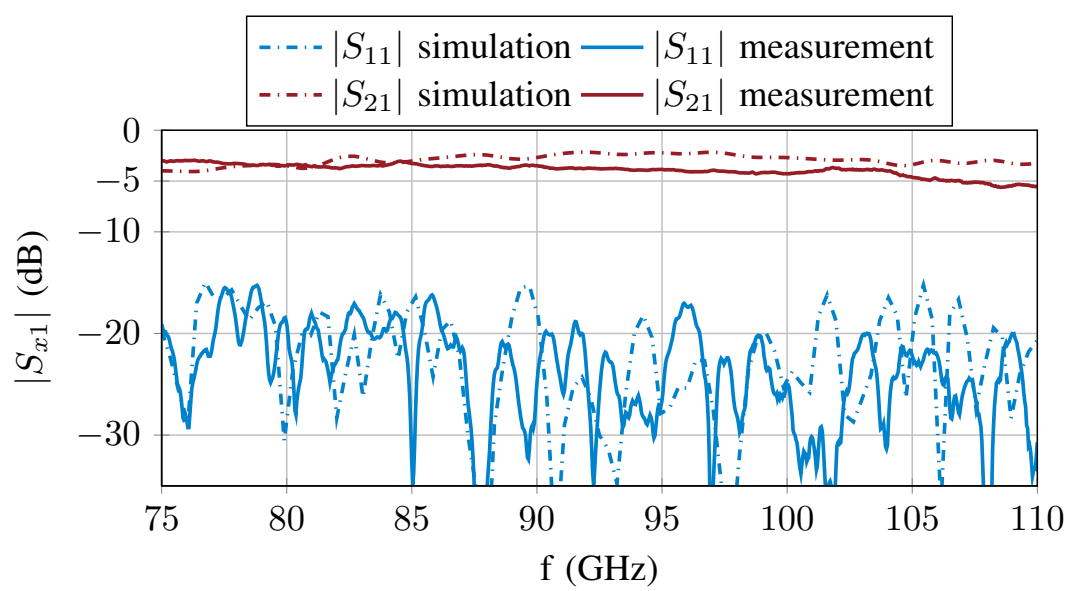

Figure 12. Measured and simulated S-parameters for orthogonal LC orientation of the SIF phase shifter.

The deviations of the simulated and measured results are caused by the assembly of the phase shifter using glue, which is not considered in the simulations [22] and tolerances which can cause radiation loss. The reflection coefficient is below $-15 \mathrm{~dB}$ over the whole $\mathrm{W}$-band. Furthermore, a differential phase shift of $230^{\circ}$ to $420^{\circ}$ and a FoM of $80^{\circ} / \mathrm{dB}$ to $105^{\circ} / \mathrm{dB}$ was achieved, see Figure 13 .

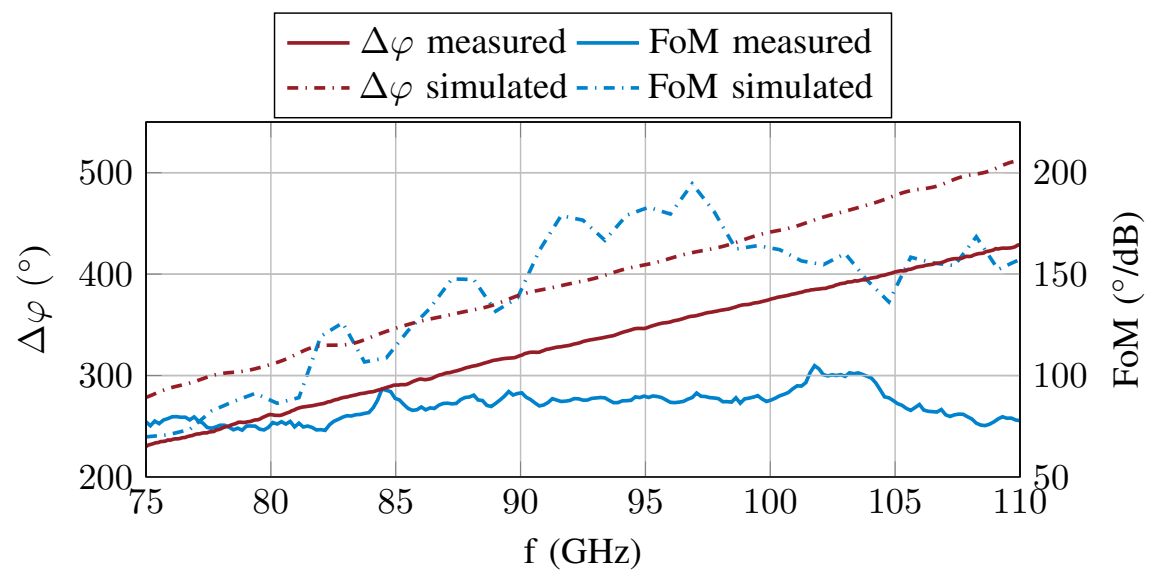

Figure 13. Measured and simulated differential phase shift and FoM of the SIF phase shifter.

As for the sWL LC phase shifter, the measured FoM and differential phase shift are less than simulated, due to non perfect LC alignment, which is assumed in the simulations. Moreover, a maximum steering efficiency of $\tau_{\varphi}=22.1 \% \mathrm{~mm}$ was obtained. 


\subsubsection{Dielectric Image Line}

A dielectric LC image line was presented in [23]. It consists of a metallic ground plane and a dielectric slab including the LC cavity with a length of $21 \mathrm{~mm}$. Figure 14 shows the topology together with the biasing concept for parallel and orthogonal orientation. The electrode system consists of four electrodes placed below and above the LC cavity. By applying a voltage to the upper electrodes, parallel orientation is obtained, see right configuration in Figure 14. For orthogonal biasing, a quadrupole field is generated, see left configuration in Figure 14. However, the bias field is not perfectly homogeneous for orthogonal orientation, due to the bended field lines. Hence, not the full anisotropy of the LC can be used. For the design of the electrode system, the main challenge was the implementation of the lower electrode lines which have to placed between dielectric and ground plane.

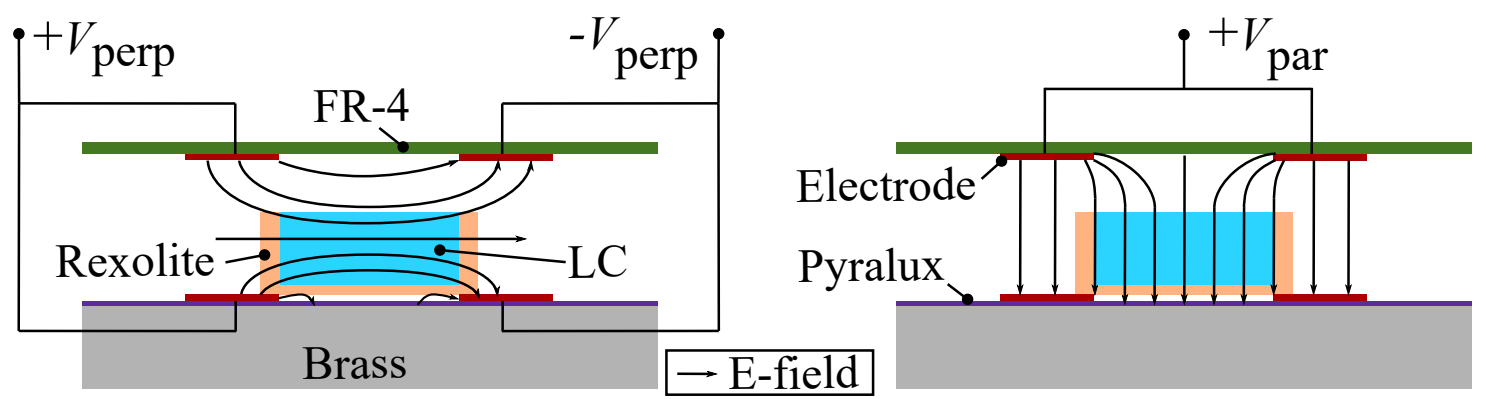

Figure 14. Cross section of the DIL phase shifter [23]. On the left and right side the bias configuration for orthogonal and parallel orientation is shown, respectively.

To guarantee isolation between electrode and ground plane, a $12 \mu \mathrm{m}$ thick Pyralux substrate with $9 \mu \mathrm{m}$ copper cladding is used. Since the electrodes are interacting with the RF field, parasitic mode excitation inside the Pyralux substrate must be prevented. Hence, the electrodes are designed with an inverted stepped impedance structure to prevent unwanted mode excitation, see Figure 15.

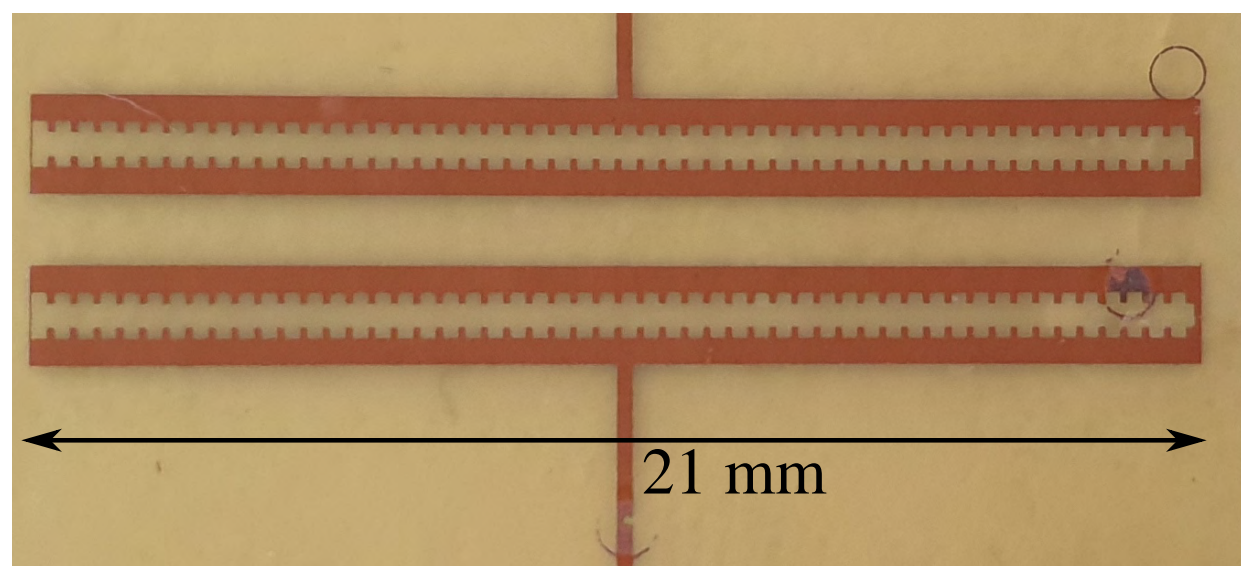

Figure 15. Design of the two lower electrodes of the DIL phase shifter with an inverted stepped impedance structure to prevent parasitic mode excitation.

In contrast, the upper electrodes are not structured, since they are placed with a certain distance above the LC cavity and have no impact on the RF performance. For the measurements, a WR-10 transition is designed and fabricated in split-block technology, see Figure 16, more details are given in [23]. The top electrode is processed on a simple FR-4 board and is placed above the DIL by using spacers. The LC cavity is filled with GT7-29001. 


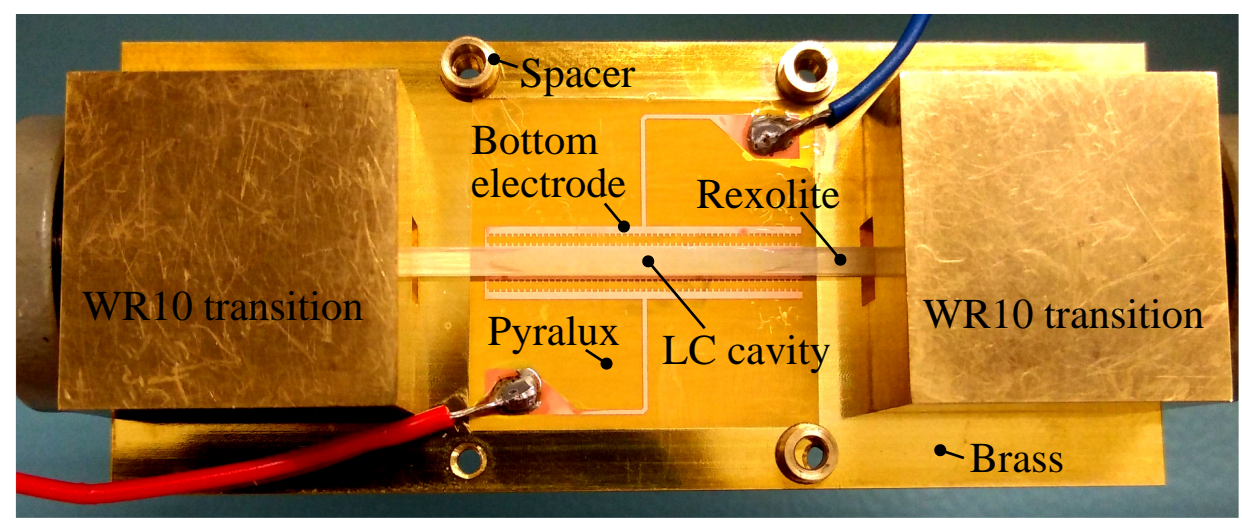

Figure 16. Photograph of the fabricated and partially assembled LC DIL phase shifter, presented in [23]. The upper electrode is placed over the LC cavity using spacers on the side.

Figure 17 shows the S-parameters for orthogonal LC orientation in comparison with simulation results. It can be seen that good matching was obtained, but the transmission coefficient is around $1 \mathrm{~dB}$ lower as in the simulations. A reason for this can be fabrication tolerances, which are causing radiation losses, and an imperfect alignment of the DIL.

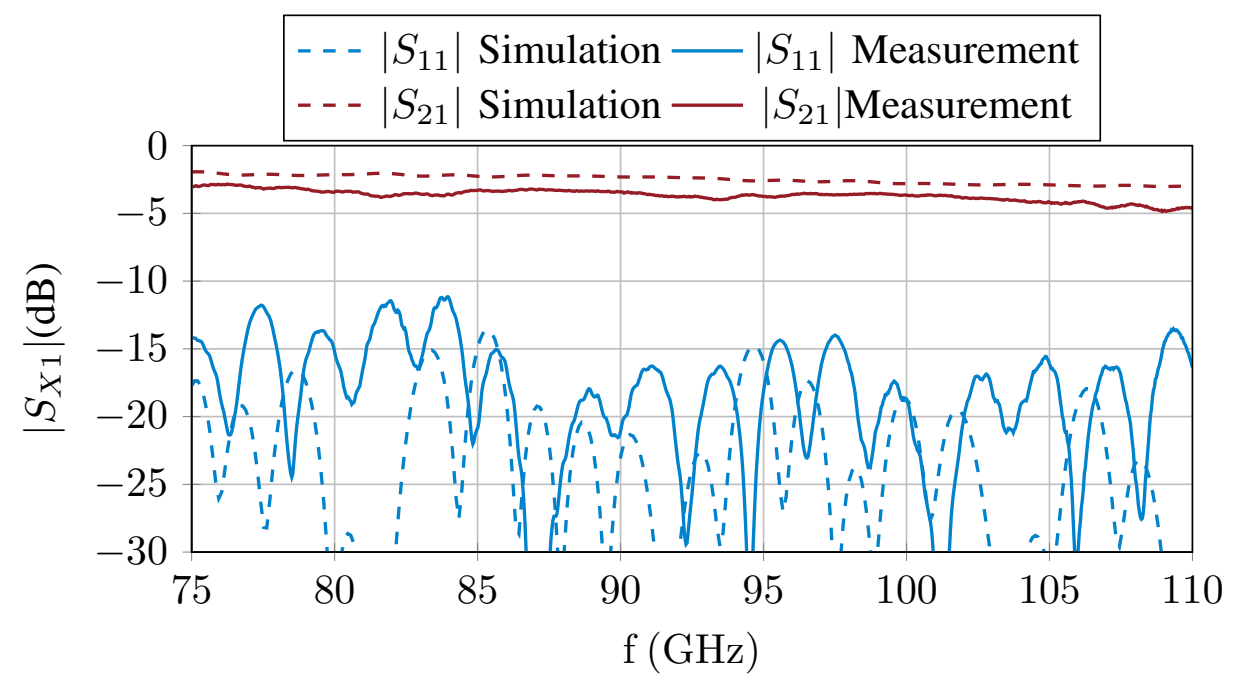

Figure 17. Measured and simulated S-parameter for orthogonal LC orientation of the DIL phase shifter.

A differential phase shift from $150^{\circ}$ to $350^{\circ}$ was obtained with a rectangular AC bias voltage of $V_{p p}=300 \mathrm{~V}$ with $1 \mathrm{kHz}$, as shown in Figure 18 . However, there is a difference of around $85^{\circ}$ between measurement and simulation. As described in detail in [23], an air gap between ground plane and DIL is the reason for this effect. Furthermore, the previously mentioned electrode system can not use the whole anisotropy range of the $\mathrm{LC}$ as in the simulations. As a consequence of the lower differential phase shift, the FoM is lower as predicted and is ranging between $50 \% / \mathrm{dB}$ to $80 \%$ dB. The maximum steering efficiency is $\tau_{\varphi}=16.7^{\circ} / \mathrm{mm}$. 


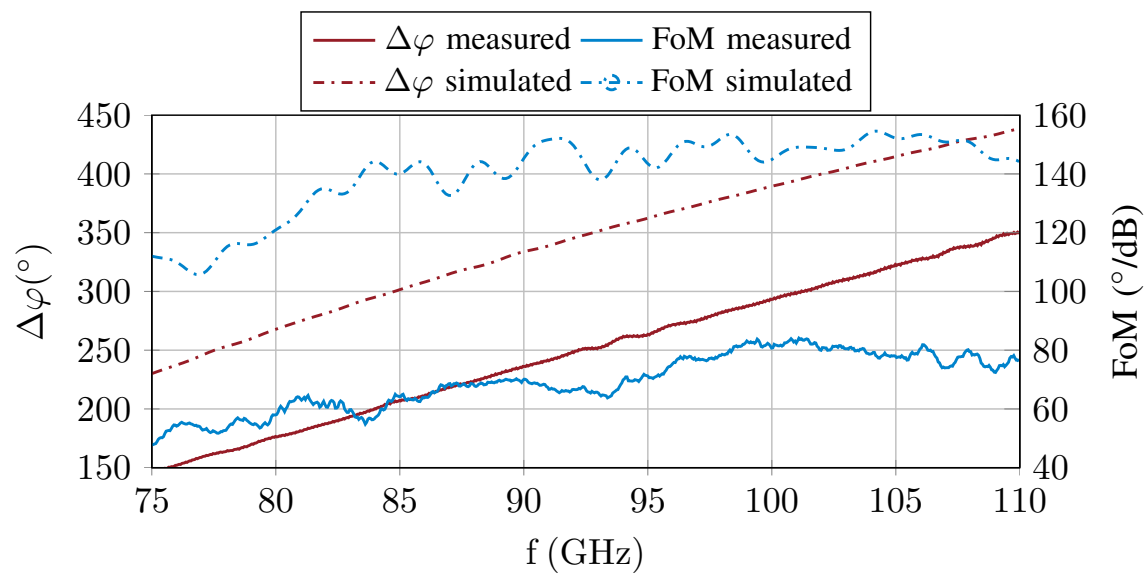

Figure 18. Measured and simulated differential phase shift and FoM applying a differential voltage of $300 \mathrm{~V}$ of the DIL phase shifter.

\subsubsection{Parallel Plate Dielectric Waveguide}

A PPDW phase shifter was designed in the V-band in [24]. As shown in Figure 19, the LC is inserted in the dielectric slab, which is placed between two parallel metal plates. Furthermore, a transition from WR-15 waveguide to PPDW was designed, which is discussed in depth in [24]. As dielectric material, Rexolite is utilized. For electrical biasing, an electrode system is integrated inside the PPDW. Therefore, $25 \mu \mathrm{m}$ thin Rogers Ultralam 3850HT $\left(\varepsilon_{r}=3.14, \tan \delta=0.002\right)$ substrates are placed between dielectric slab and metal plane, by gluing them onto the metal plates. For full LC orientation three electrode strips are designed on each side. Since the electrodes are directly placed on the dielectric slab, they have an influence on the RF performance. Hence, an inverted stepped impedance structure is used to prevent parasitic mode excitation in the substrates, as shown in Figure 20a. In Figure 20b,c the bias configuration for parallel and orthogonal orientation of the LC molecules is presented, respectively. The PPDW phase shifter is fabricated using a modular system, as depicted in Figure 21. Therefore, the WR-15 transitions are milled out of brass as single parts in split-block technology. The two metal plates are laser cut out of stainless steel and are mounted between the transitions afterwards. To mill the LC cavity inside the slab, two Rexolite halves are fabricated which are glued together using UV adhesive. Afterwards, the LC cavity is filled with the LC mixture GT5-26001.

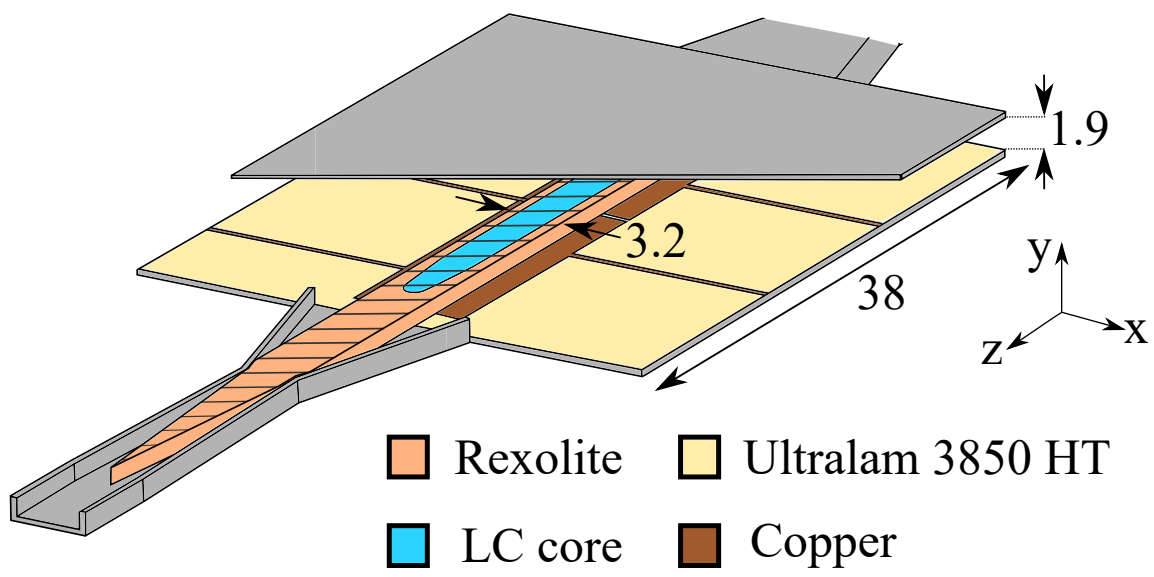

Figure 19. Cross section of the LC PPDW phase shifter with transition to WR15 waveguide, as presented in [24]. All dimensions are given in $\mathrm{mm}$. 


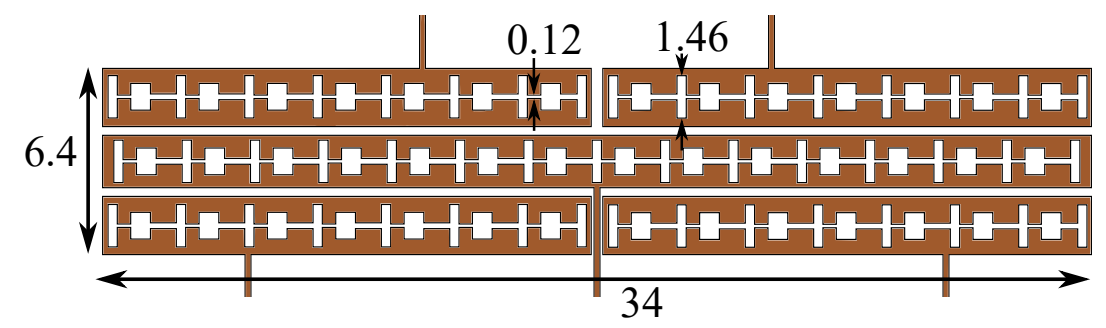

(a)

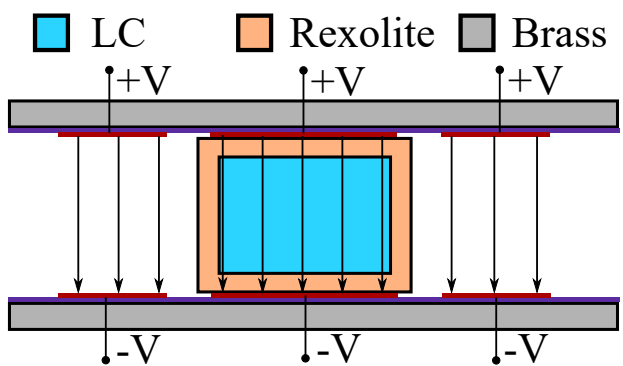

(b)

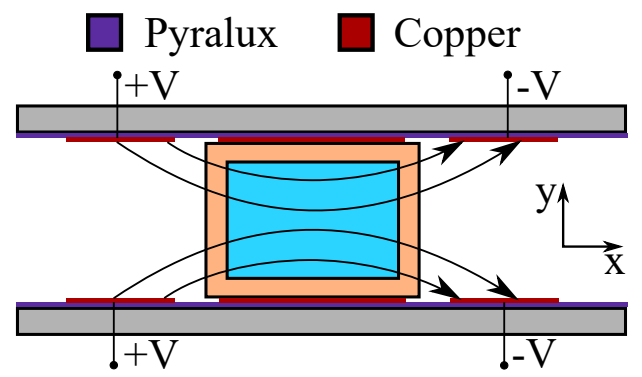

(c)

Figure 20. (a) Electrode design of the PPDW phase shifter with an inverted stepped impedance structure for mode suppression. All dimensions are given in $\mathrm{mm}$. $(\mathbf{b}, \mathbf{c})$ show the bias configurations for parallel and orthogonal LC orientation, respectively.

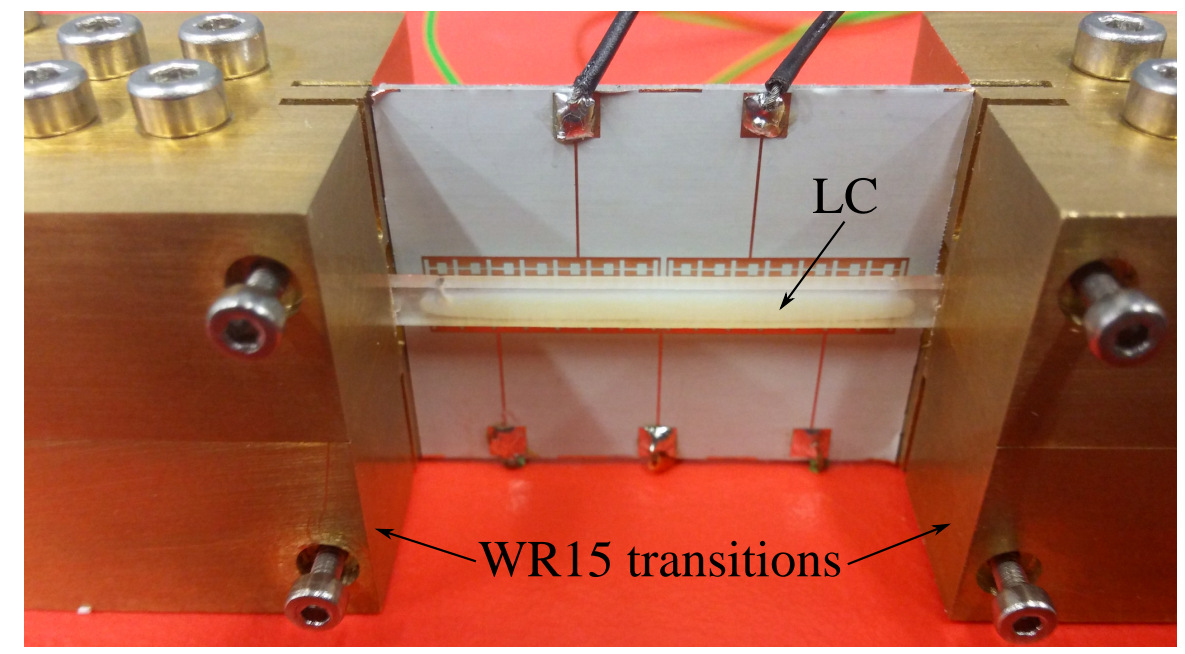

Figure 21. Photograph of the partially assembled PPDW phase shifter.

For the measurements a PNA-X from Keysight Technology is used to measure from $50 \mathrm{GHz}$ to $65 \mathrm{GHz}$ and a PNA with W-band extensions to measure over the whole V-band up to $75 \mathrm{GHz}$. For LC biasing, a rectangular $1 \mathrm{kHz}-\mathrm{AC}$ bias voltage of $V_{p p}=300 \mathrm{~V}$ is applied. The measured S-parameters, shown in Figure 22, are compared to simulation results. It can be seen that the reflection coefficient fits very well with the simulation, whereas the transmission coefficient is around $0.8 \mathrm{~dB}$ to $1.3 \mathrm{~dB}$ lower as simulated. A reason for this can be the modular assembly, since small gaps between metal plates and transitions have arisen. 


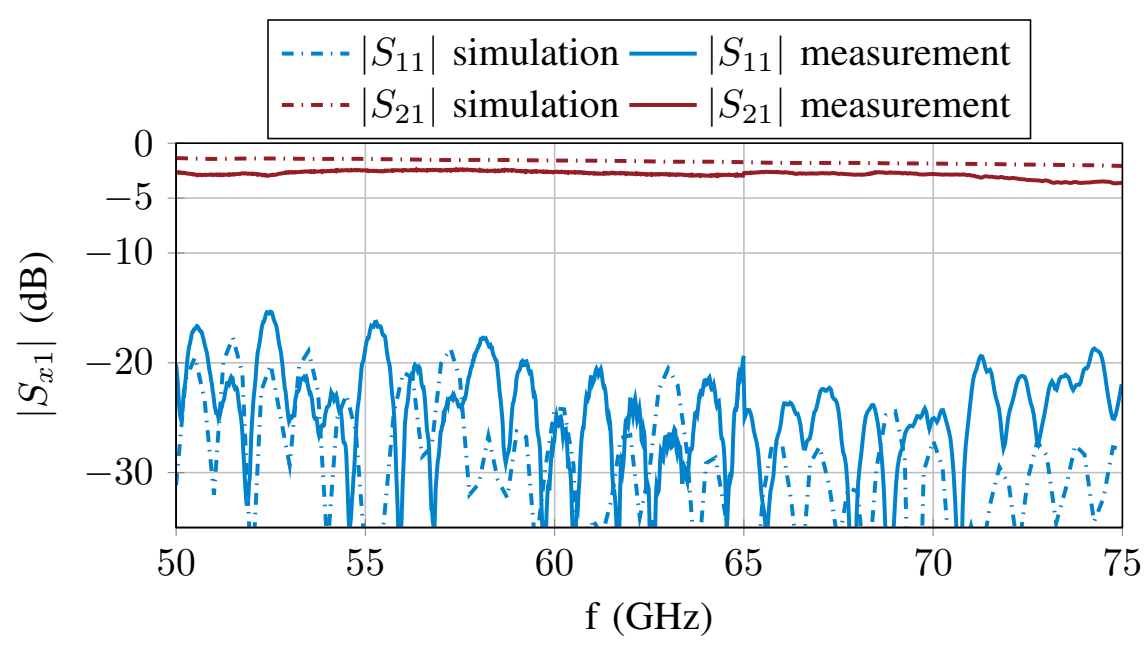

Figure 22. Simulated and measured S-parameters of the PPDW phase shifter.

The resulting differential phase shift and FoM are shown in Figure 23. Due to the bias configuration for orthogonal LC biasing, compare Figure 20c, the bias field is not perfectly orthogonal to the RF field. Therefore, the LC molecules are not perfectly aligned, which results in a reduction of the usable anisotropy and with that in a reduced differential phase shift. A maximum FoM and steering efficiency for electrical biasing of $113 \% / \mathrm{dB}$ and $10 \% \mathrm{~mm}$ is achieved, respectively.

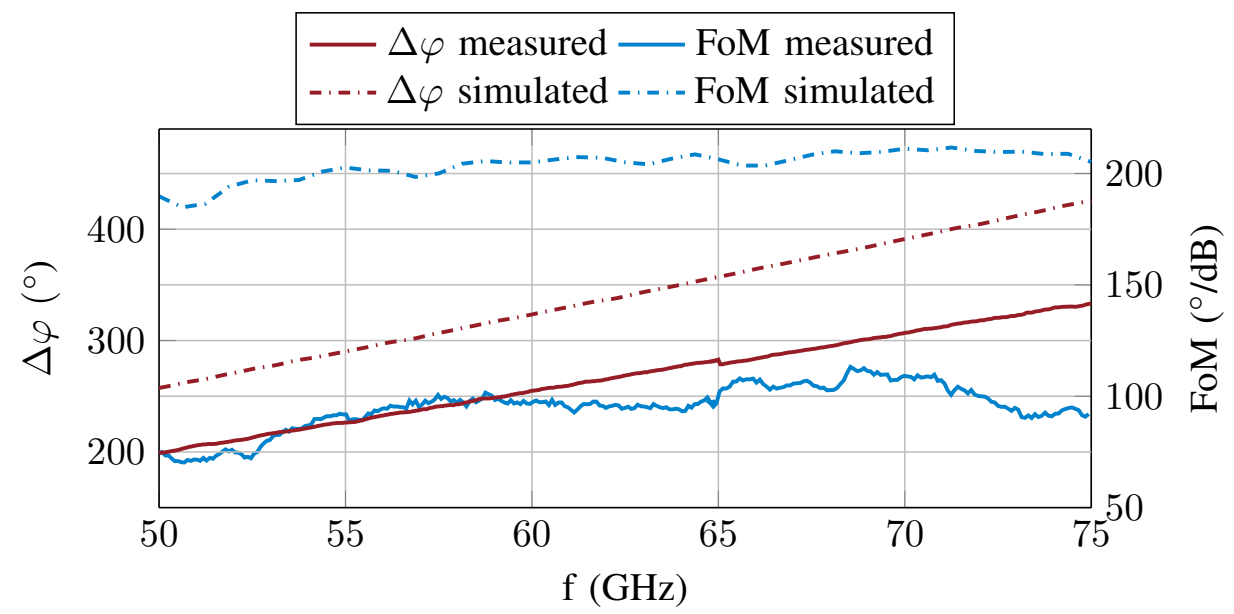

Figure 23. Differential phase shift and FoM of the PPDW phase shifter.

\subsubsection{Nonradiative Dielectric Waveguide}

The setup and cross section of the NRD and PPDW LC phase shifter are similar, however the dimensions and the operating mode are different. In contrast to the PPDW a higher order hybrid mode is used for operation. Moreover, some design rules have to be taken into account, to guarantee the nonradiative characteristic of the NRD. Therefore, the plate separation and the cross section have to be chosen in such a way, that the NRD can be operated at the desired frequency band. A more detailed overview of the design process can be found in [25]. The electrode system and bias configuration are identical to the PPDW phase shifter. However, the NRD phase shifter is designed at W-band, therefore, the dimensions of the electrodes and stepped impedance structure are smaller and are processed on a thin Pyralux substrate, as shown in Figure 24. 


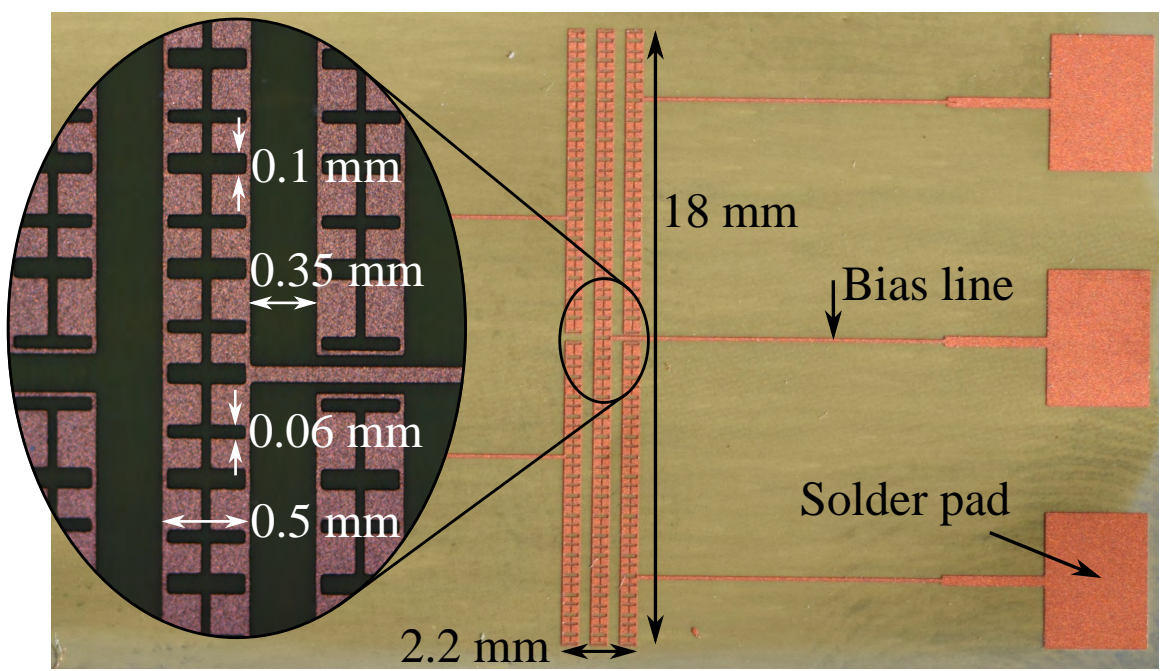

Figure 24. Photograph of the Pyralux substrate with the processed electrodes for the NRD phase shifter.

The LC cavity has a length of $18 \mathrm{~mm}$, all dimensions are given in [26]. For measurements, a WR-10 transition is designed and in contrast to the PPDW phase shifter, it is fabricated out of one piece in split-block technology, see Figure 25. The Rexolite slab with the LC cavity is fabricated in two halves, which are glued together with UV adhesive.

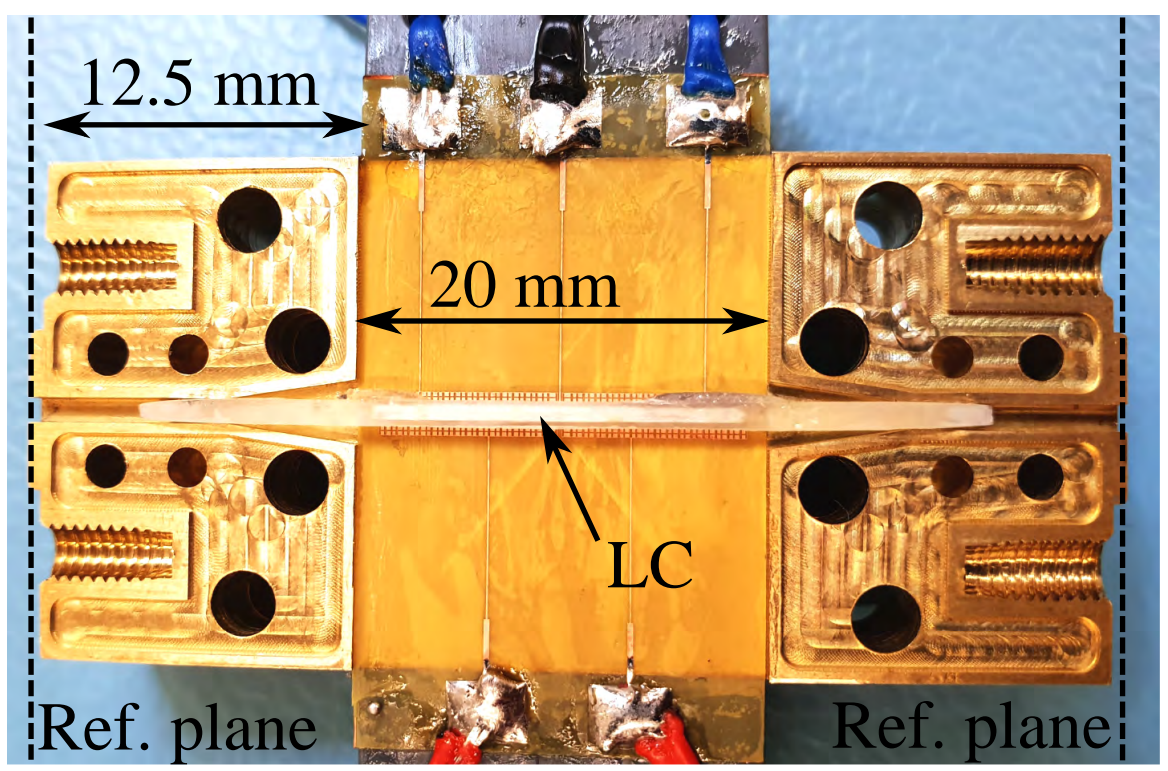

Figure 25. Photograph of the partially assembled NRD phase shifter.

Due to the bandwidth limitation of the NRD, the phase shifter is designed for the upper W-band from $98 \mathrm{GHz}$ to $110 \mathrm{GHz}$. An $1 \mathrm{kHz}-\mathrm{AC}$ bias voltage of $300 \mathrm{~V}$ is applied for LC orientation. The simulation and measurement results of the S-parameters with GT5-26001 are depicted in Figure 26. The difference between simulated and measured transmission coefficient is ranging from $1 \mathrm{~dB}$ to $2 \mathrm{~dB}$, which are higher as for the PPDW phase shifter. A main reason for this is that the NRD has smaller dimensions, which makes it more prone to fabrication tolerances. Furthermore, accurate gluing of the Rexolite halves is more challenging. 


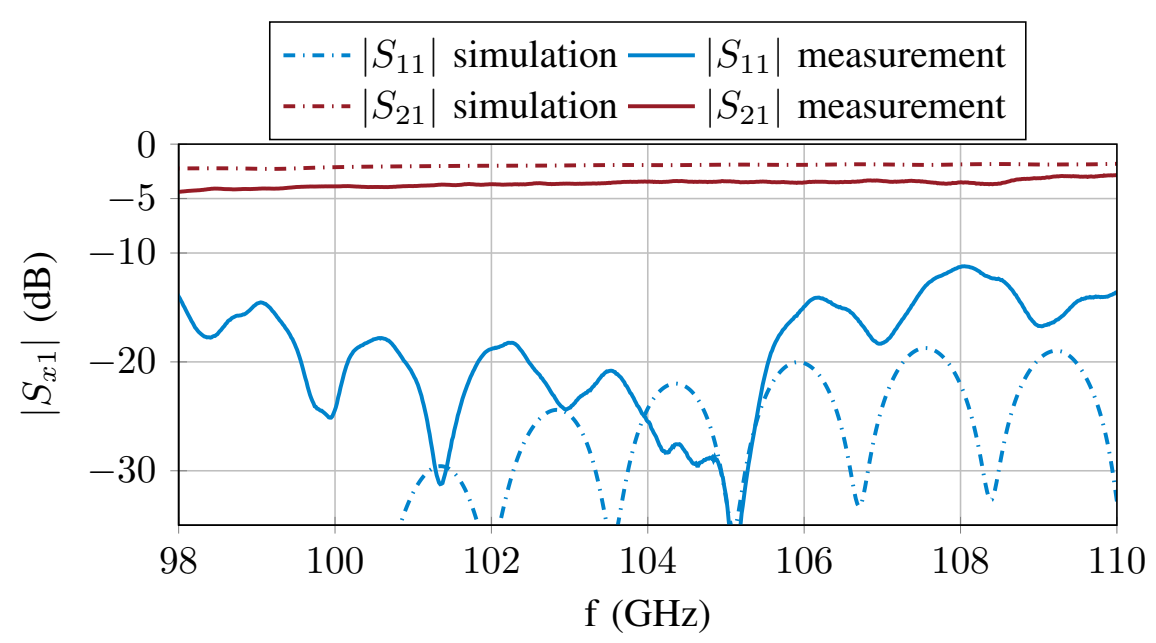

Figure 26. Simulated and measured S-parameters of the NRD phase shifter.

Similar to the PPDW, the measured differential phase shift is around $40^{\circ}$ less as simulated and in ranging between $240^{\circ}$ to $270^{\circ}$, as shown in Figure 27.

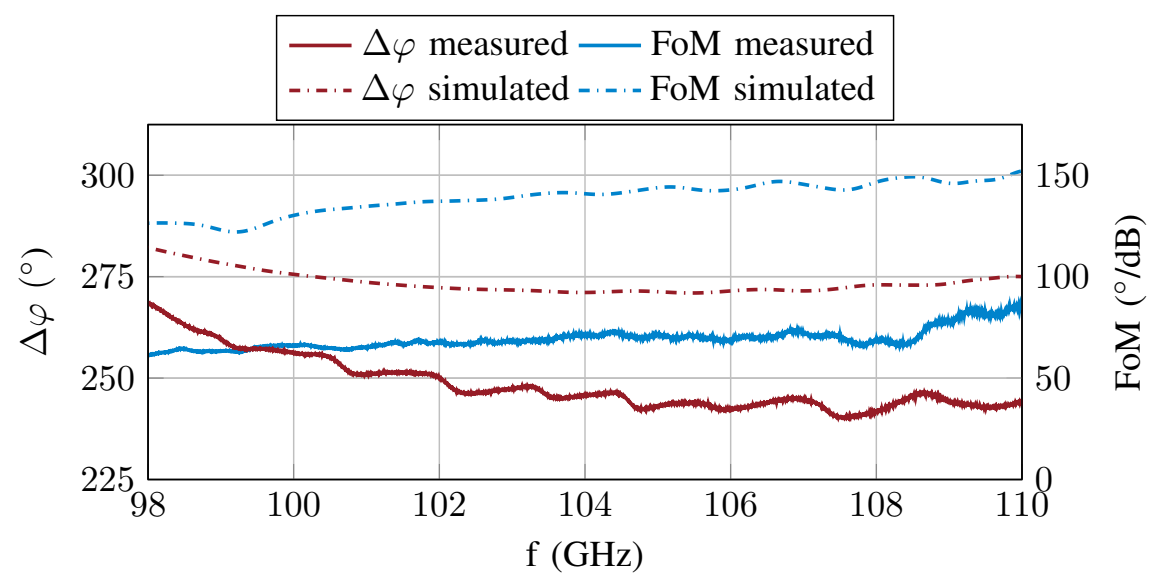

Figure 27. Simulated and measured differential phase shift and FoM of the NRD LC phase shifter.

Moreover, a maximum FoM and steering efficiency of $85^{\circ} / \mathrm{dB}$ and $15^{\circ} / \mathrm{mm}$ was obtained.

\subsection{Comparison of Different Tunable Dielectric Waveguides}

The various topologies of LC phase shifters introduced in this section are summarized in Table 2. Due to the technical advancement in microwave LC technology, different LC mixtures are used throughout the development of dielectric LC based components. As the slightly differing material parameters require special adjustment regarding RF design, an exact one-to-one comparison is not possible. In addition, all proposed phase shifter topologies depend on individual waveguide transitions from a hollow waveguide topology. As these transitions are included in every phase shifter measurement, they influence the achieved FoMs in a negative way, meaning that higher FoMs could be achieved when the transitions are neglected. Nevertheless, Table 2 provides a good overview of topologies and their potential. The choice of topology is application dependent. If the FoM is to be maximized, the sWL is a promising topology, however, if faster response time and integration with other circuitry is of higher priority, topologies as the DIL or the NRD are are candidates to choose from. 
Table 2. Summary of the tunable LC phase shifter results based on dielectric waveguide topologies.

\begin{tabular}{llccc}
\hline Topology & LC Mixture & $f(\mathbf{G H z})$ & FoM $_{\max }\left({ }^{\circ} / \mathbf{d B}\right)$ & $\tau_{\varphi}(\mathrm{dB} / \mathbf{m m})$ \\
\hline sWL & GT7-29001 & 75 to 110 & 130 & 27.3 \\
SIF & GT5-26001 & 75 to 110 & 105 & 22.1 \\
DIL & GT7-29001 & 75 to 110 & 80 & 16.7 \\
PPDW & GT5-26001 & 50 to 75 & 113 & 10 \\
NRD & GT5-26001 & 98 to 110 & 85 & 15 \\
\hline
\end{tabular}

\subsection{Antennas}

In this section LC based millimeter wave antennas are reviewed which are candidates for future wireless communications. The spatial radiation characteristic, the antenna's far-field pattern, is depending on the type of the used antenna element and, if an array structure is present, the geometrical arrangement of the antenna elements. The influence which is solely depending on the type of antenna used, such as aperture (horn) or resonator (dipole) antennas, is called the element factor EF. The contributions which are due to the geometrical arrangement in an antenna array are called the array factor AF. Therefore, for a single antenna AF $=1$. Assuming a linear concatenation of $N$ arbitrary antenna elements with distance $d$ to each other, the corresponding two-dimensional far field in $\theta$ direction represented by

$$
E_{\theta}=\mathrm{EF} \cdot \sum_{n=0}^{N-1} I_{n} \cdot e^{j\left(n k d \cos \left(\theta+\alpha_{n}\right)\right.}=\mathrm{EF} \cdot \mathrm{AF}
$$

$k$ represents the wave number and is therefore frequency dependent, as well as $\alpha_{n}$ which denotes the individual phase at each antenna element. Therefore, phase shifters which have direct influence on $\alpha_{n}$ enable the manipulation of the far-field characteristic of an antenna array. The LC-based phase shifters introduced in the previous section can serve this purpose. Some applications are shown in the first part of this section. While in applications, the antenna arrays have to show a high number of antenna elements, we focus on demonstrators with less elements, which are more feasible for lab demonstrations. Besides antenna arrays, a novel LC based approach where the EF instead of the AF is modified for beam steering with a single antenna is covered at the end of the section. These antennas are called lateral wave beam steering antennas. It prove to be beneficial both for antenna arrays and lateral wave antennas to reduce the amount of metal involved in the antenna topology to a minimum.

\subsubsection{Phased Array Antennas}

The LC-based phased array concept has been put to use at frequencies up to $30 \mathrm{GHz}$ in planar, metallic topologies [27-29]. In this subsection, the development from metallic approaches to fully dielectric approaches at $\mathrm{W}$-band are presented, in order to provide practical verification of the aforementioned advantages of dielectric topologies.

First demonstrators up to $\mathrm{K}_{\mathrm{a}}$-band $(26.5 \mathrm{GHz}$ to $40 \mathrm{GHz})$ have been realized in a hollow-waveguide environment [30-32]. Figure 28 shows a model of the proposed $4 \times 4$ antenna array of [30]. Power division is obtained by Y-dividers in a corporate feed arrangement. The radiating elements are realized as metallic horn antennas, which are connected to a phase shifter each. While a non-reconfigurable array (no phase shifters present) in this topology is easy to realize, the introduction of phase shifters proved to be challenging. Details have been discusses already in Section 2.1. Therefore, a metal-reliant system should be omitted, especially when aiming for higher frequencies. As a direct consequence, a hybrid approach is presented in [20], where the phase shifters and antennas are fully dielectric. The phase shifters are realized in sWL topology (see Section 2.1.1) filled with GT7-29001. The antennas are realized as simple dielectric rods. Figure 29 depicts the schematic of the $1 \times 4$ array demonstrator. While the power division is still handled in a hollow waveguide environment with Y-dividers (A), the phase shifters and the antennas are realized in a fully dielectric manner ( $\mathrm{C}$ and 
D). All dielectric components are milled from Rexolite 1422. In order to ensure an efficient change from metallic to fully dielectric environment without high radiation loss, a transition region (B) is necessary. In this region, the electric field is coupled to the dielectric material, and short horn transitions mitigate radiation loss when leaving the metal environment. With the transition to a fully dielectric environment, a phase shifter with easy access to electrodes and low loss can be employed in the sWL topology. Figure 30a shows the simulated S-parameters of the divider, which ensures that a quarter $(\approx-6 \mathrm{~dB})$ of the input power at Port 1 is incident at Port 2 to Port 5 of the divider, while reflections are low. The phase shifters are biased by simple copper plates on which different potentials are assigned. Figure $30 \mathrm{~b}$ depicts a cross-sectional view of the four $33 \mathrm{~mm}$ long phase shifters and the electrodes. Only electrodes at the sides of the phase shifters are used in the setup of [20]. Therefore, 5 instead of 13 bias electrodes are necessary. As a result, a certain bias state can be set up, but for a reset to the initial perpendicular LC molecule state no control is present and one has to rely on natural realignment over time. By assigning different potentials to the electrodes, different effective permittivities are evoked in the phase shifters. As radiation elements dielectric rod antennas are chosen. Dielectric rod antennas are broadband antenna elements which are easy to fabricate $[33,34]$, since they are simply tapered dielectric waveguides.

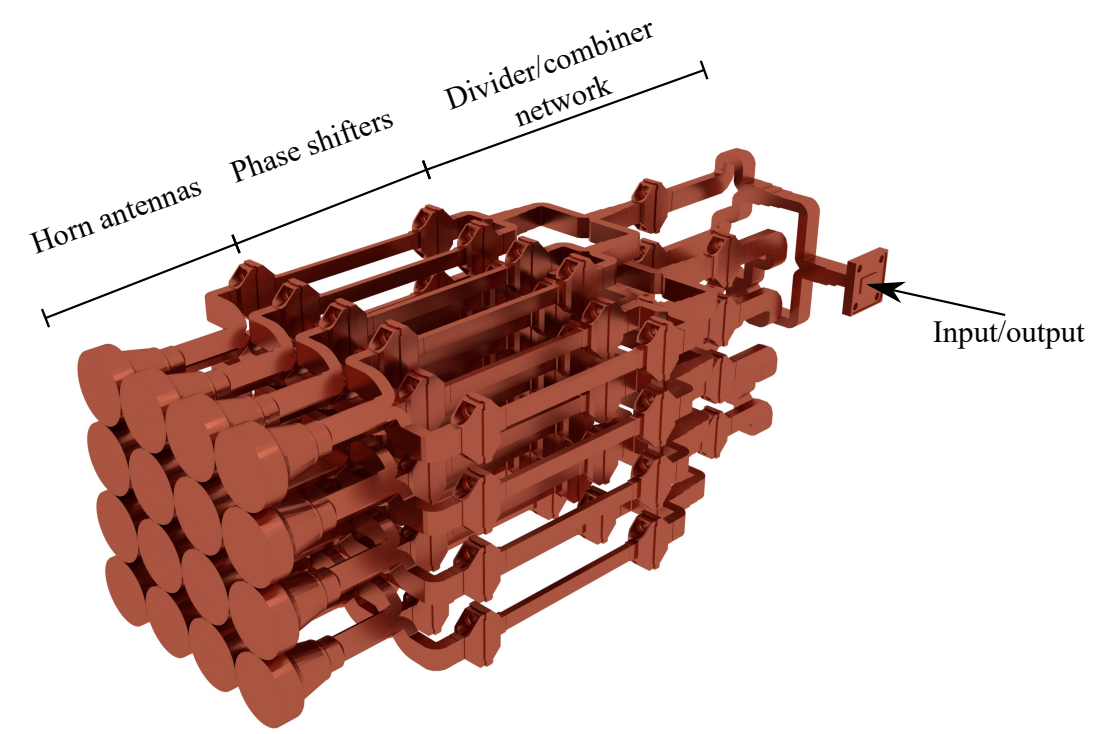

Figure 28. Model of the $4 \times 4$ antenna array proposed in [30].

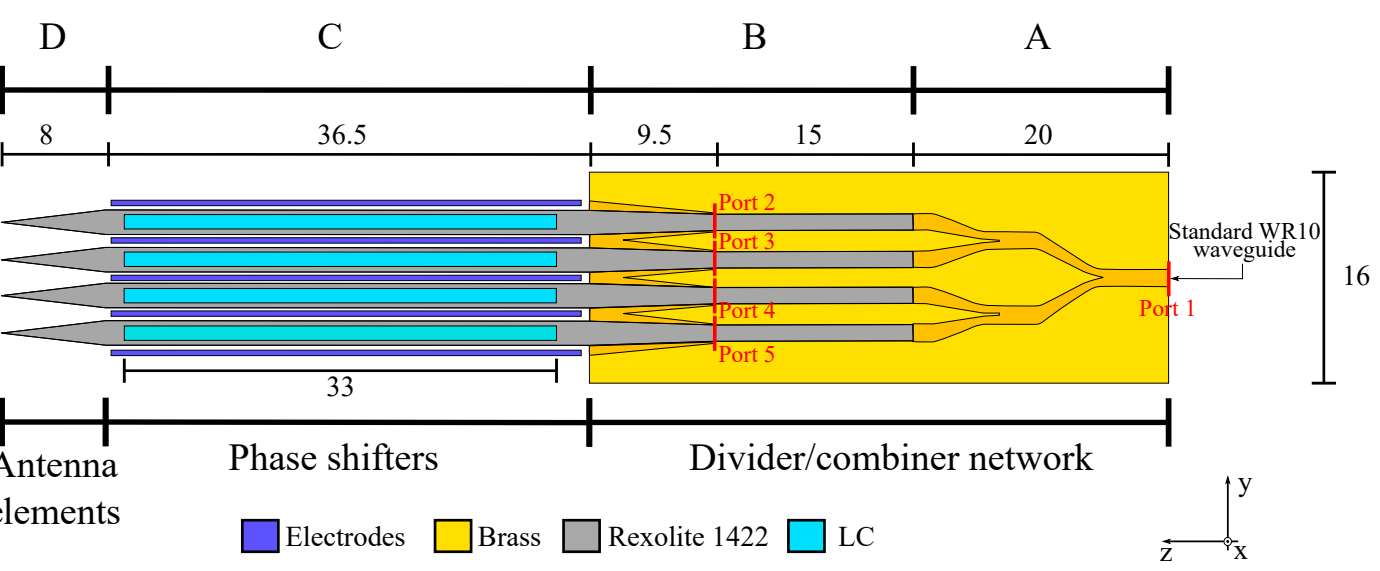

Figure 29. Schematic of the hybrid antenna array proposed in [20]. All dimensions given in mm. 


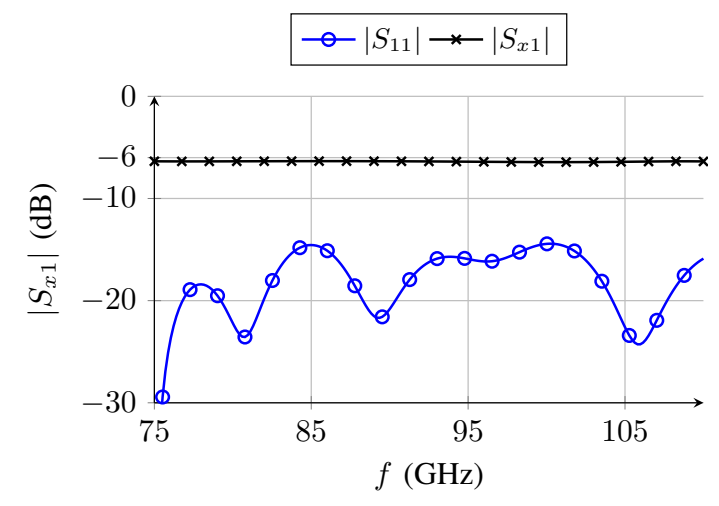

(a) Simulated S-parameters of the divider network. Port1 as source, $x=2, \ldots, 5$.

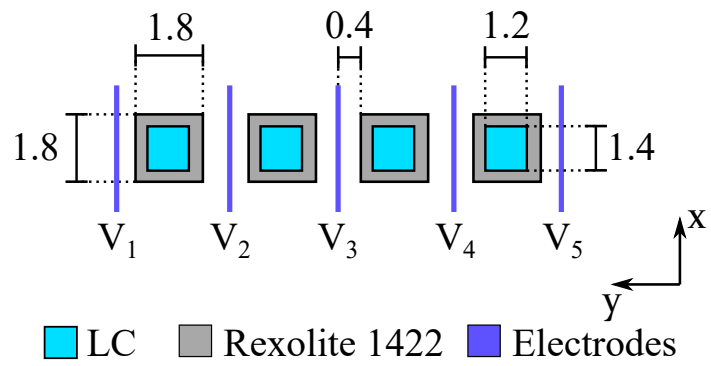

(b) Cross section on the phase shifters and the bias electrodes.

Figure 30. (a) Simulation results of the divider network, (b) bias structure of the array. For steering, different voltages $V_{1}$ to $V_{5}$ with respect to a common ground are applied to bias the LC differently in the individual branches. All dimensions given in $\mathrm{mm}$.

Due to the taper, a propagating wave is gradually decoupled from the dielectric to the surrounding medium (air). As these rod antennas are fully dielectric, they are light-weight, and very attractive for mass-production. The assembled array is depicted in Figure 31a. Good matching below $-10 \mathrm{~dB}$ is maintained throughout the entire W-band, compare Figure 31b. Three different beam configurations with radiation towards $0^{\circ}, 15^{\circ}$, and $-25^{\circ}$ are demonstrated in [20]. The acquired E-Plane pattern are depicted in Figure 32. While the direction of the main beam is as desired, ripples and high side lobes are observable, which are not present in simulations. The main reason for these phenomena is the highly modular setup of the antenna array [20]. Fabrication tolerances in each of sections A-D (see Figure 29) can cause small errors to accumulate. In addition, the phase shifters had to be glued, but the influence of glue is not considered in the simulations.

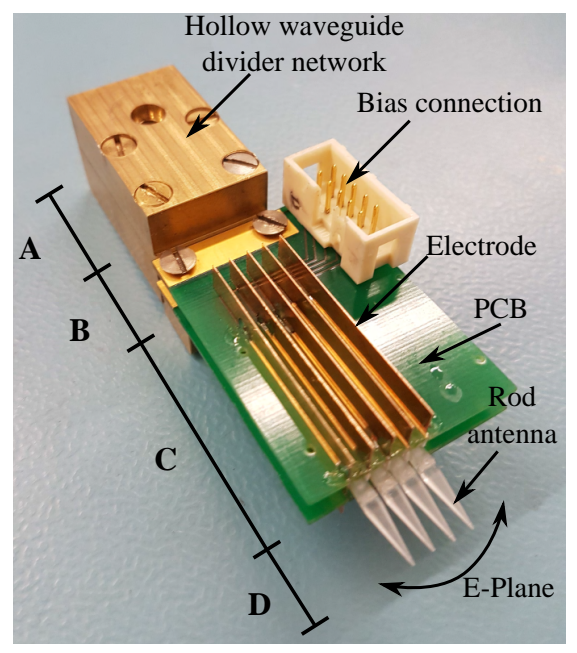

(a)

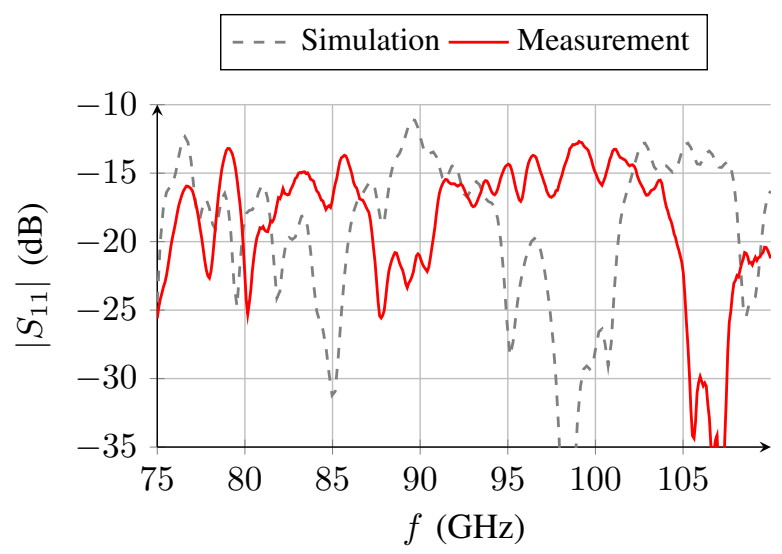

(b)

Figure 31. (a) Fully assembled demonstrator with PCB for maintaining electrode position and bias connection, Sections A to D according to Figure 29. (b) Simulation and measurement of the input reflection. 


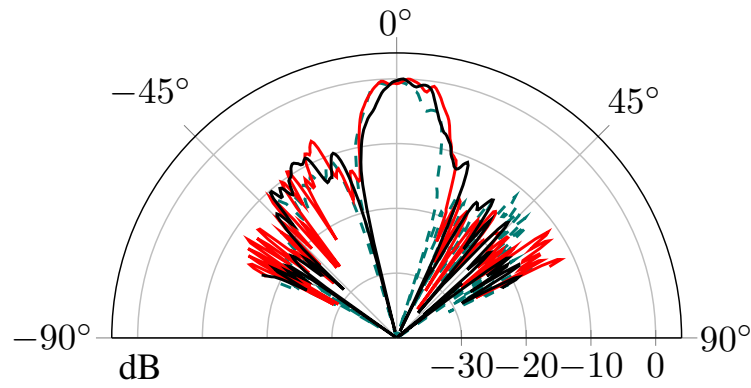

(a) $\Theta_{\text {beam }}=0^{\circ}$, measurement.

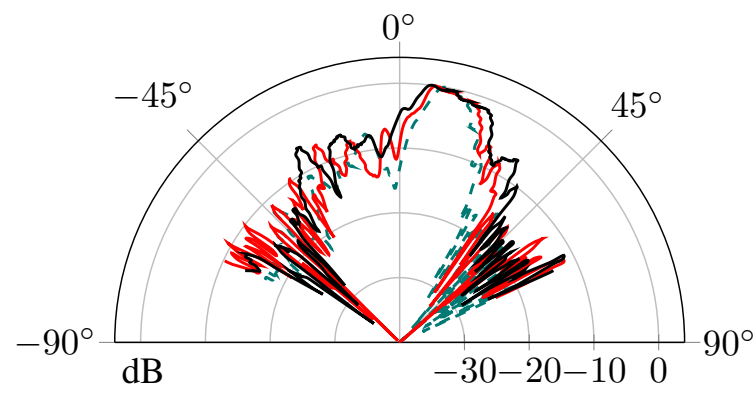

(c) $\Theta_{\text {beam }}=15^{\circ}$, measurement.

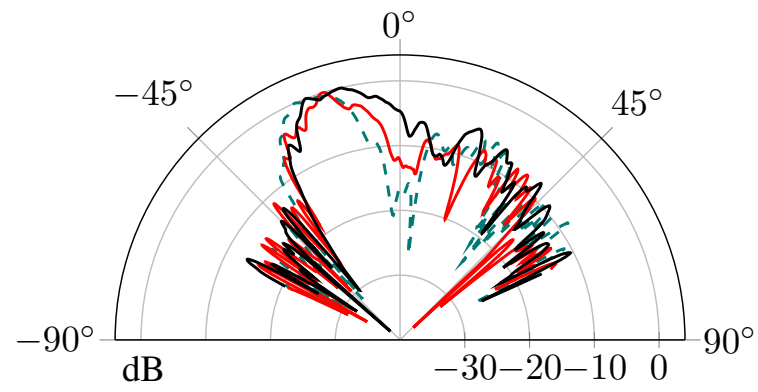

(b) $\Theta_{\text {beam }}=-25^{\circ}$, measurement.

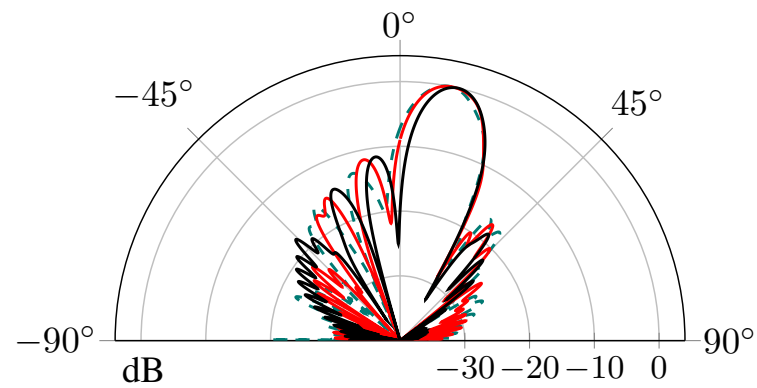

(d) $\Theta_{\text {beam }}=15^{\circ}$, simulation.

$$
\text { - - - } 85 \mathrm{GHz}-95 \mathrm{GHz}-100 \mathrm{GHz}
$$

Figure 32. Pattern at three different frequencies, $(\mathbf{a}, \mathbf{b})$ are measured results, while $(\mathbf{c}, \mathbf{d})$ serves as a reference to the simulations.

In order to circumvent the modular approach which can be the cause for accumulating small errors, a fully dielectric design is presented in [35]. The main difference to [20] is the power divider network. Instead of classical Y-dividers, a concept from optics, multimode-interference (MMI), is utilized. MMI is well described by [36] and is often referred to as self-imaging, since the input field distribution repeats itself at distinct distances in the multimode waveguide. This phenomenon has its origins in the different propagation constants of each mode in a multimode waveguide. In order to ensure that multiple modes can form in a dielectric waveguide, it has larger physical dimensions than a sWL. Figure 33 depicts the field distribution in a multimode waveguide fed by a sWL from the right. Both the multimode waveguide and the singlemode sWL have the same height $h=1.8 \mathrm{~mm}$ and permittvity $\varepsilon_{r}=2.53$ but differ in width. The singlemode feed has a width of $w_{\mathrm{sm}}=1.8 \mathrm{~mm}$ and the multimode section has a width of $w_{\mathrm{mm}}=12.25 \mathrm{~mm}$. Distinct field maxima, images of the input field distribution, can be observed at precise distances from the feeding point.

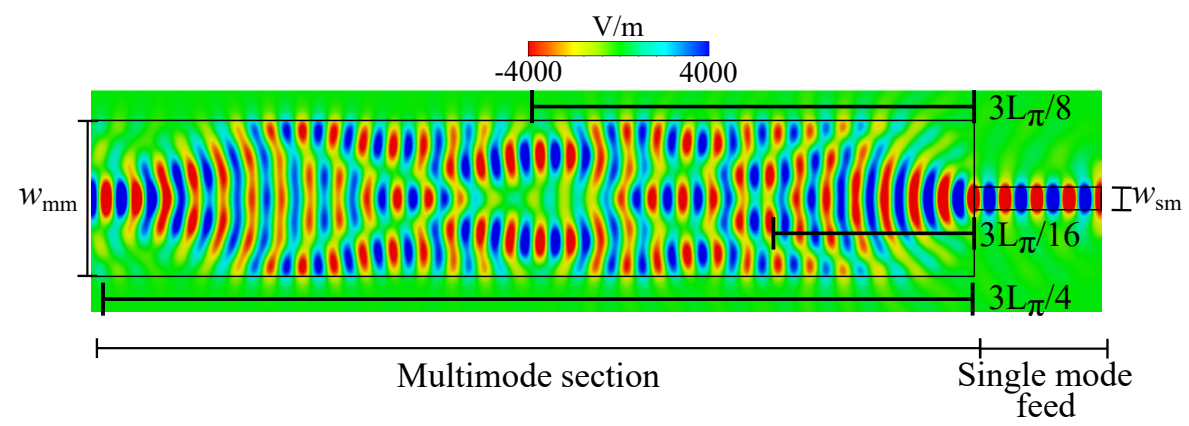

Figure 33. Field distribution in a multimode waveguide centrally fed by a single mode waveguide at $92.5 \mathrm{GHz} . w_{\mathrm{sm}}=1.8 \mathrm{~mm}, w_{\mathrm{mm}}=12.25 \mathrm{~mm}, \varepsilon_{r}=2.53$. Relevant lengths for explicit number of maxima are highlighted. 
The number of maxima, their position and phase to each other and their respective power varies with distance from the feed. Following the considerations in [36,37], with a feed centrally placed at the multimode section, the distance for the appearance of $N$ images is described by

$$
L_{N}=\left(3 L_{\pi}\right) /(4 N),
$$

where $L_{\pi}$ describes the beat length of the multimode section. The beat length can be determined by

$$
L_{\pi}=\frac{4 \sqrt{\varepsilon_{r}} w_{\mathrm{eff}}^{2}}{3 \lambda_{0}}
$$

with $\varepsilon_{r}$ denoting the relative permittivity of the dielectric. $w_{\text {eff }}$ represents the effective width of the multimode section and $\lambda_{0}$ is the free space wavelength of operation. Since parts of the wave are propagating outside the dielectric, the effective width has to be taken into account in Equation (7). $L_{\pi}$ can be controlled by the physical width $w$ of the device when designing the power divider. If the length of the multimode-section is set to $L_{N}=L_{4}$, for example, four maxima appear at the end of the section, which can be coupled to four different sWLs. These waveguides can then feed the actual antenna element. Even though the MMI power divider is band-limited due to its frequency dependency, operation bandwidths of around $15 \mathrm{GHz}\left(\approx 15 \%\right.$ with center frequency $\left.f_{c}=97.5 \mathrm{GHz}\right)$ can be achieved. The first non-reconfigurable millimeter wave antenna array based on MMI has been proposed in [38], and its beam steering capabilities have been demonstrated in [39]. It is possible to extend the MMI-concept to two-dimensional cases [40], and if the dielectric constant of the dielectric material is increased, the antenna array can be further decreased in size [41]. An overview of fully dielectric non-configurable antenna arrays based in $\mathrm{MMI}$ at $\mathrm{mmW}$-frequencies can be found in [42]. Figure 34 depicts the layout of a fully dielectric $1 \times 4$ antenna array including LC phase shifters [35]. A singlemode waveguide feeds the MMI section, which divides the power equally to the connected waveguides which include LC phase shifters, realized in the sWL topology. The antenna elements are formed by simple rod antennas. Note the different taper lengths in order to compensate phase difference introduced by the MMI-concept. Compared to the hollow-waveguide approach the MMI divider provides a significant reduction of the power division section $(18 \mathrm{~mm}$, Figure 34 , instead of $44.5 \mathrm{~mm}$, Figure 29). In addition, the MMI-divider is lighter, is not assembled by different pieces and is fully dielectric, which means that no transitional region from metallic to dielectric waveguides is necessary. Figure 35 depicts a full-wave simulation of the corresponding antenna.

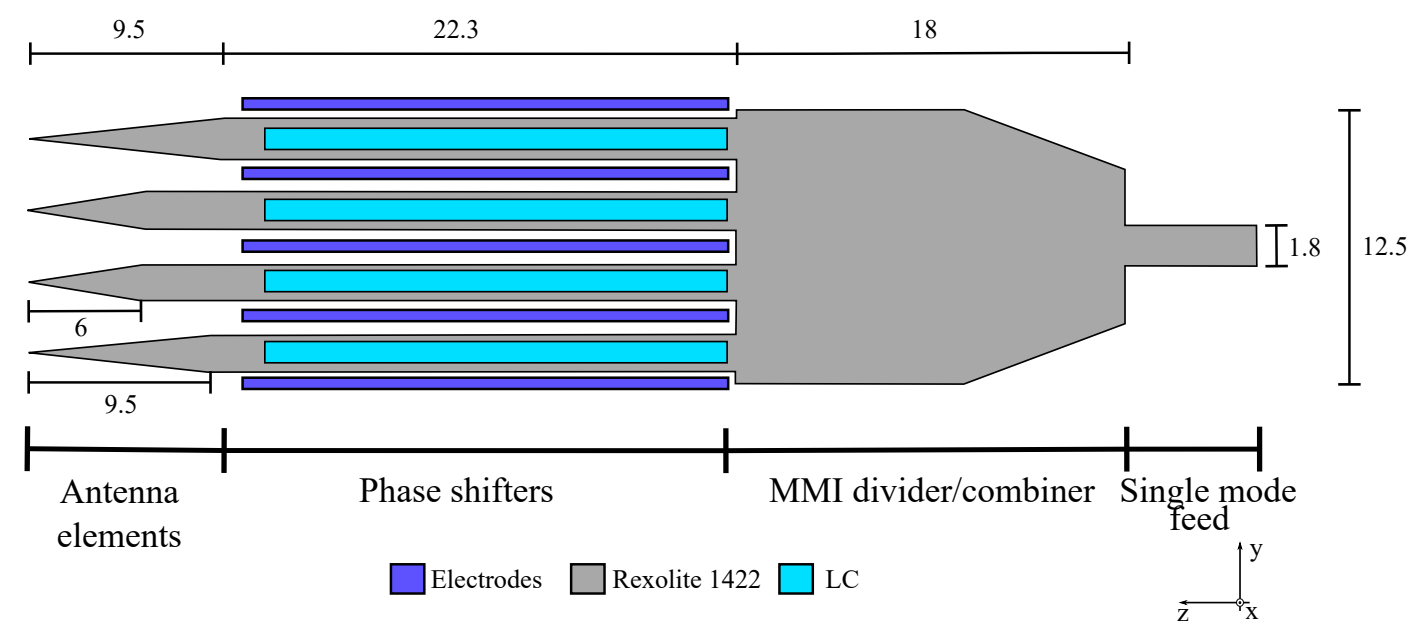

Figure 34. Layout of the fully dielectric $1 \times 4$ reconfigurable antenna array. All dimensions in mm [35]. 


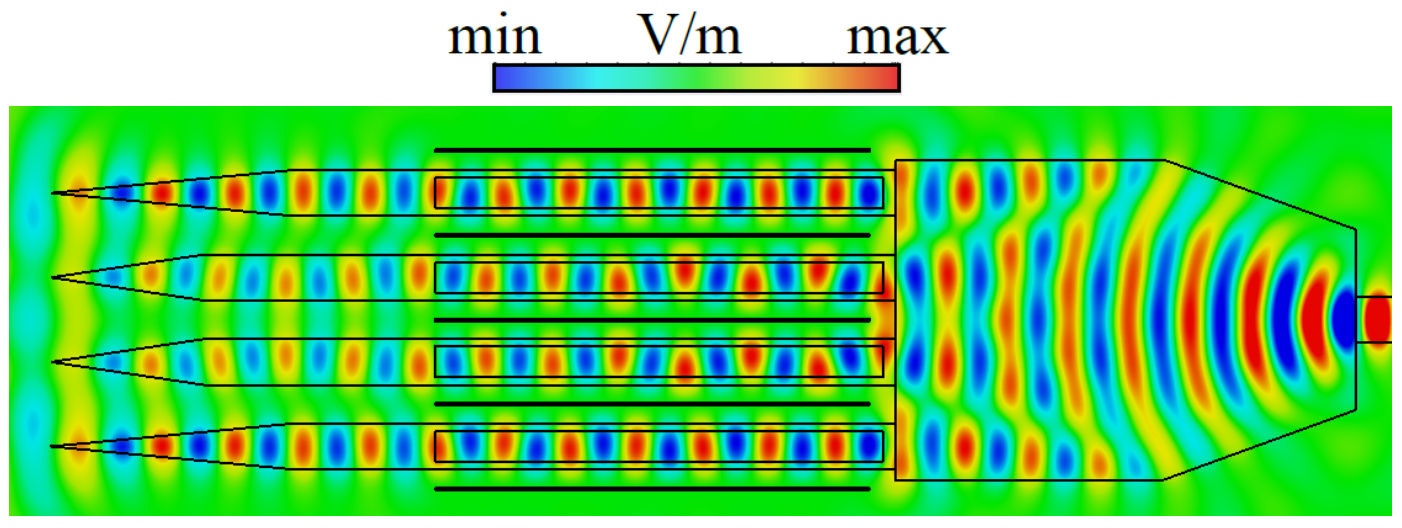

Figure 35. Field simulation of the MMI-fed antenna array at $90 \mathrm{GHz}$.

The array body, milled as one piece from Rexolite 1422, is shown in Figure 36a. Only the rod antennas have to be connected to the array structure with small Rexolite pins. The fully assembled demonstrator is shown in Figure 36b.

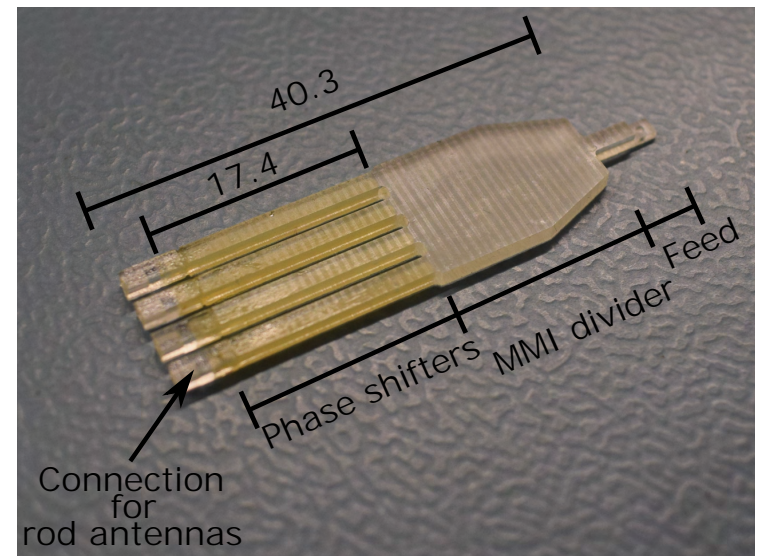

(a)

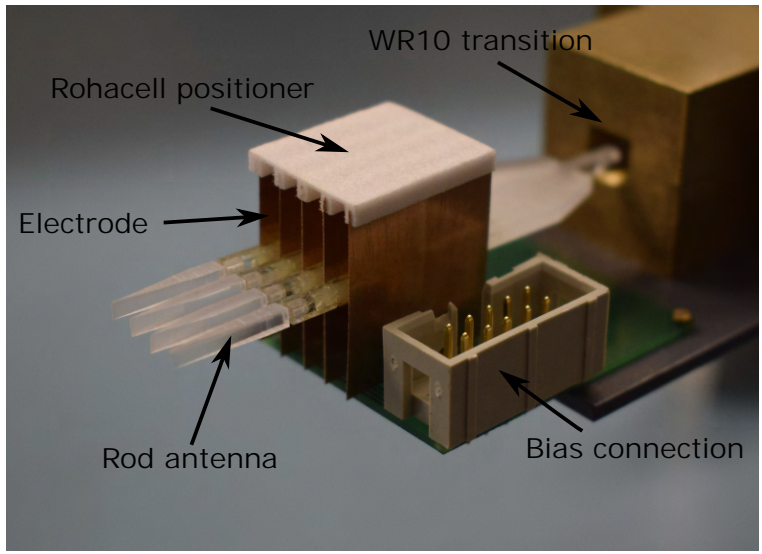

(b)

Figure 36. (a) Array body milled from one piece with filled and sealed LC phase shifters, (b) fully assembled demonstrator with bias electrodes, rod antennas and a transition from WR-10 to dielectric waveguide. Dimensions given in $\mathrm{mm}$.

The necessary electrodes are held in place by a small PCB at the bottom with corresponding bias supply. For more stability, a small piece of Rohacell HF51 [43] is used. As in [20], the demonstrator utilizes only electrodes for the perpendicular LC molecule alignment and relies on relaxation effects in the bias-free state. In order to avoid glued LC cavities, which can cause additional loss, this design relies on cavities directly drilled into the dielectric rods. Since the drill length is limited, the phase shifter section is $17.4 \mathrm{~mm}$ long and therefore about two times shorter than the phase shifter in [20]. The hole diameter is $d_{\text {drill }}=1.4 \mathrm{~mm}$. Therefore, less differential phase shift of maximal $240^{\circ}$ is possible, which results in less demonstrable steering angle. Good matching of the whole antenna array with $\left|S_{11}\right|<-10 \mathrm{~dB}$ is visualized in Figure 37. The corresponding patterns acquired with different bias configurations are depicted in Figure 38. 


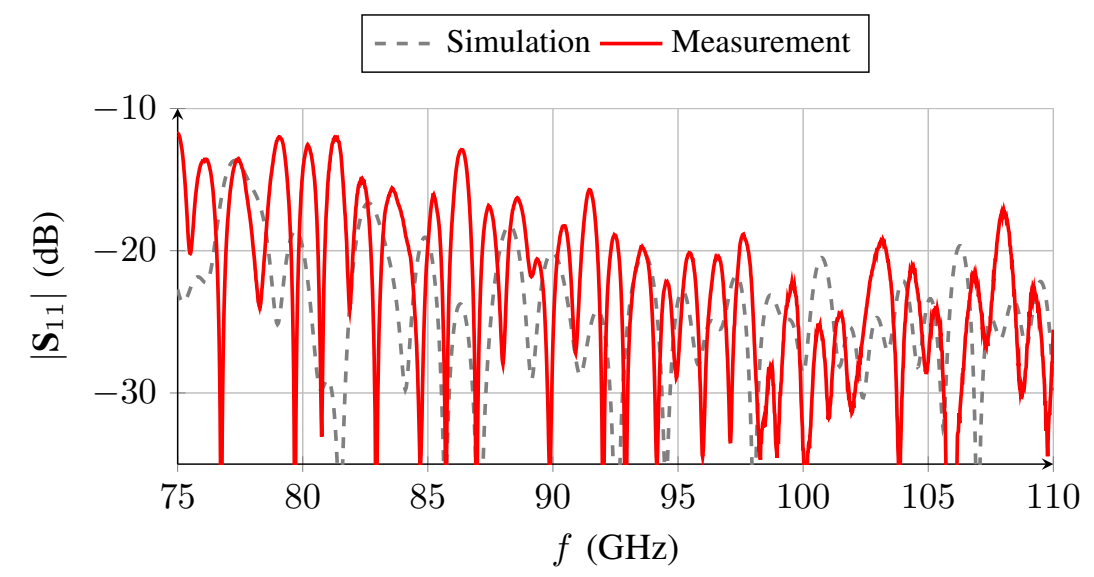

Figure 37. Simulated and measured input reflection of the antenna array.

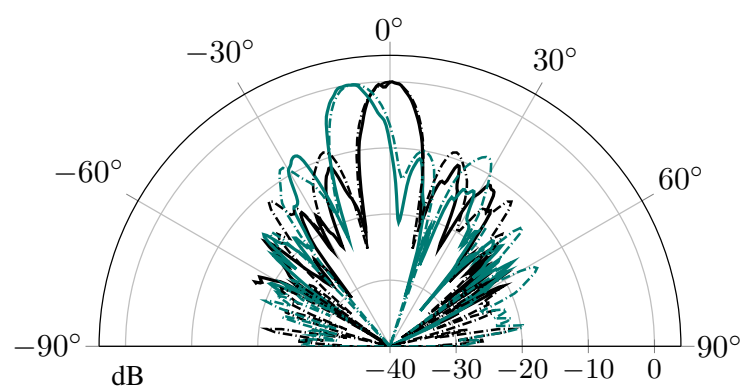

(a) $90 \mathrm{GHz}$

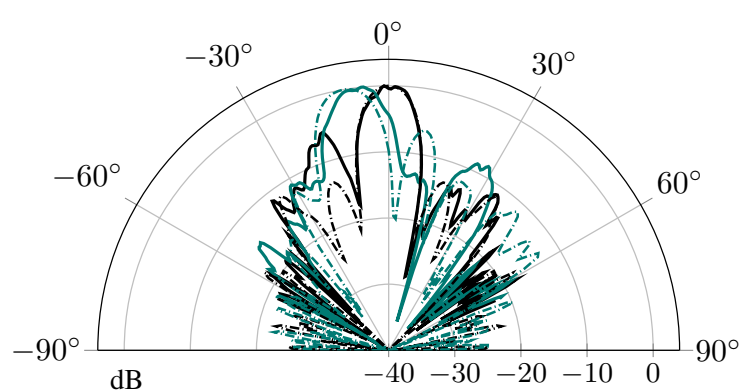

(b) $95 \mathrm{GHz}$

$$
\begin{aligned}
& \text { - - - Simulation } 10^{\circ}-\text { Measurement } 10^{\circ} \\
& \text { - - Simulation } 0^{\circ}-\text { Measurement } 0^{\circ}
\end{aligned}
$$

Figure 38. Simulated and measured pattern of the fully dielectric antenna array at different frequencies.

Compared to the patterns in Figure 32 we can see more clear main lobes and no ripples in the main lobe. This is mostly due to less single parts which have to be linked, since the whole antenna body is milled from one piece. Overall, the fully dielectric antenna shows enhanced performance, while being easier to fabricate and having less size and weight. However, due to the shorter phase shifters, the demonstrator only shows steering angles of $\pm 10^{\circ}$. Nevertheless, simulation and measurement are much better aligned than in [20].

This section showed the potential of a fully dielectric design with the whole device milled from one piece where possible. The absence of metal reduces the assembly effort, disturbed radiation and weight of the antenna arrays. An industrialized mass production is easily possible for large scale application of the antennas.

\subsubsection{Lateral Wave Beam Steering Antennas}

The antennas covered in this section do not rely on the phased array concept. They belong to the family of lateral wave antennas [44] and solely guide the wave along the antenna body towards the desired direction of radiation. Hence instead of the array factor AF, the element factor EF is manipulated in order to achieve beam steering. The first LC-based antenna of this kind was realized in parallel-plate topology [45]. Figure 39 depicts the underlying concept of beam steering, represented by a ray-tracing approach. A semicircle of lower permittivity, $\varepsilon_{r, \text { low }}$, hosts a sector of higher permittivity $\varepsilon_{r}$,high, which has an opening angle of $\varphi$. This sector always has its origin at the middle of the straight edge of the semicircle, at which a feed is located. As the sector has higher permittivity than the surrounding material, an electromagnetic wave follows the angle $\theta_{\text {sect }}$ the sector is pointing to. 
The higher the permittivity of the sector, the better the field confinement in it and therefore the higher $\theta_{\text {sect }}$ can get. If $\theta_{\text {sect }}$ exceeds a critical angle $\theta_{\text {crit }}$ the wave cannot be confined any more and leaks out of the sector according to Snell's law

$$
\theta_{\text {crit }}=90^{\circ}-\arcsin \left(\frac{\sqrt{\varepsilon_{r, \text { low }}}}{\sqrt{\varepsilon_{r, \text { high }}}}\right) .
$$

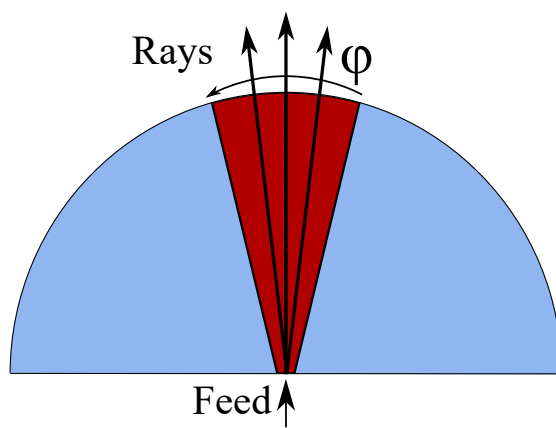

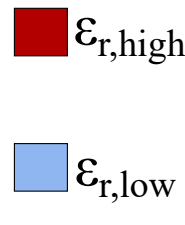

Feed $\uparrow$

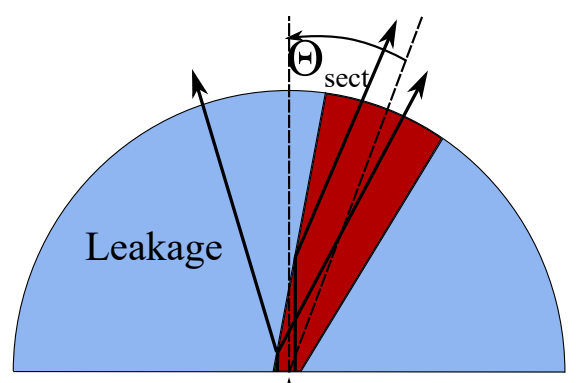

Feed $\uparrow$

Figure 39. Beam steering principle of lateral wave antennas represented by rays.

The anisotropy of LC can be used to form these areas of low or high permittivities by means of electrical bias fields. However, due to the limited anisotropy of LC, $\theta_{\text {sect }}$ is limited to a theoretical upper boundary of $\theta_{\text {sect }}=30^{\circ}$ before the critical angle is exceeded, assuming the anisotropy of GT3-23001 $\left(\Delta \varepsilon_{r}=0.77\right)$, which is used in [45]. Changing the angular opening $\varphi$ of the sector affects gain and HPBW in H-plane, as the sector represents a dielectric sectoral horn [46]. In principle, more than one sector can be employed in order to achieve a multi-beam radiation pattern [45].

Figure 40 depicts the layout of the antenna in parallel-plate topology. The LC is hosted by a sectoral cavity in a Rexolite 1422 body. The angular width of the sector is $\Phi=111^{\circ}$. The semicircular Rexolite body has a radius of $35 \mathrm{~mm}$ and is fed by a hollow waveguide to parallel-plate dielectric waveguide transition. The high permittivity region has an opening angle of $\varphi=40.5^{\circ}$. At the end of the LC cavity the host material (Rexolite 1422) has a $5 \mathrm{~mm}$ long taper. This taper ensures low reflection when the wave is decoupled from the dielectric. In order to enhance gain in E-plane, the parallel plates are flared to a metallic horn-like opening at the end of the antenna.

A field simulation of the described model is visualized in Figure 41a. Propagation of the wave to the desired direction can be observed, however, parasitic radiation is present, since Equation (8) represents a ray-approach and neglects the nature of wave propagation. This leakage leads to side lobes in the antenna pattern. In addition, the resulting beam angle is usually less than the sector steering angle $\theta_{\text {beam }} \leq \theta_{\text {sect }}$. Figure $41 \mathrm{~b}$ shows the simulated gain and side-lobe level (SLL) for three different frequencies. We can observe that gain increases with frequency, as the effective aperture of the antenna increases with smaller wavelength. The SLL increases with steering angle, since more power leaks from the high permittivity sector with increasing steering angle $\theta_{\text {sect }}$ of the high permittivity sector.

In order to form the desired high permittivity or low permittivity regions, electrodes have to be employed in between the parallel plates and the Rexolite body.

As already mentioned in the phase shifter section above, a stepped impedance structure is necessary in the parallel-plate topology such that no parasitic modes form between electrodes and surrounding metal [45]. The electrodes are strips radially extending from the feeding point, such that they can create sectors of different permittivity in the LC cavity. For continuous steering each electrode would have to be controlled individually. Since not enough high-voltage sources were present to address each electrode, the demonstrator in [45] shows three different bias configurations to switch between. With fixed bias regions, electrodes which have to show similar potentials regardless of beam configuration can be biased with the same line, hence, the design is significantly simpler. In total, five different beam configurations can be achieved, but in this manuscript we focus on the steered 
$\left(\theta_{\text {beam }} \approx 30^{\circ}\right)$ and un-steered $\left(\theta_{\text {beam }}=0^{\circ}\right)$ configuration, and refer the interested reader to [45]. Figure 42 depicts the top electrode network with assigned voltages and the corresponding normal or tangential field components which determine high or low permittivity, respectively. The bottom electrodes show same or complementary voltage distribution. Due to the close placement of the electrodes a good footprint of the bias is created in the LC cavity. However, one can see that the tangential configuration leads to small regions with no field strength present directly beneath the electrodes. In addition, the boundary between high and low permittivity is not abrupt, but shows a changeover region from high to low permittivity. This is due to overlapping field components and the continuity of LC molecule alignment. A bias voltage of $\pm 150 \mathrm{~V}$ is assumed in simulations.
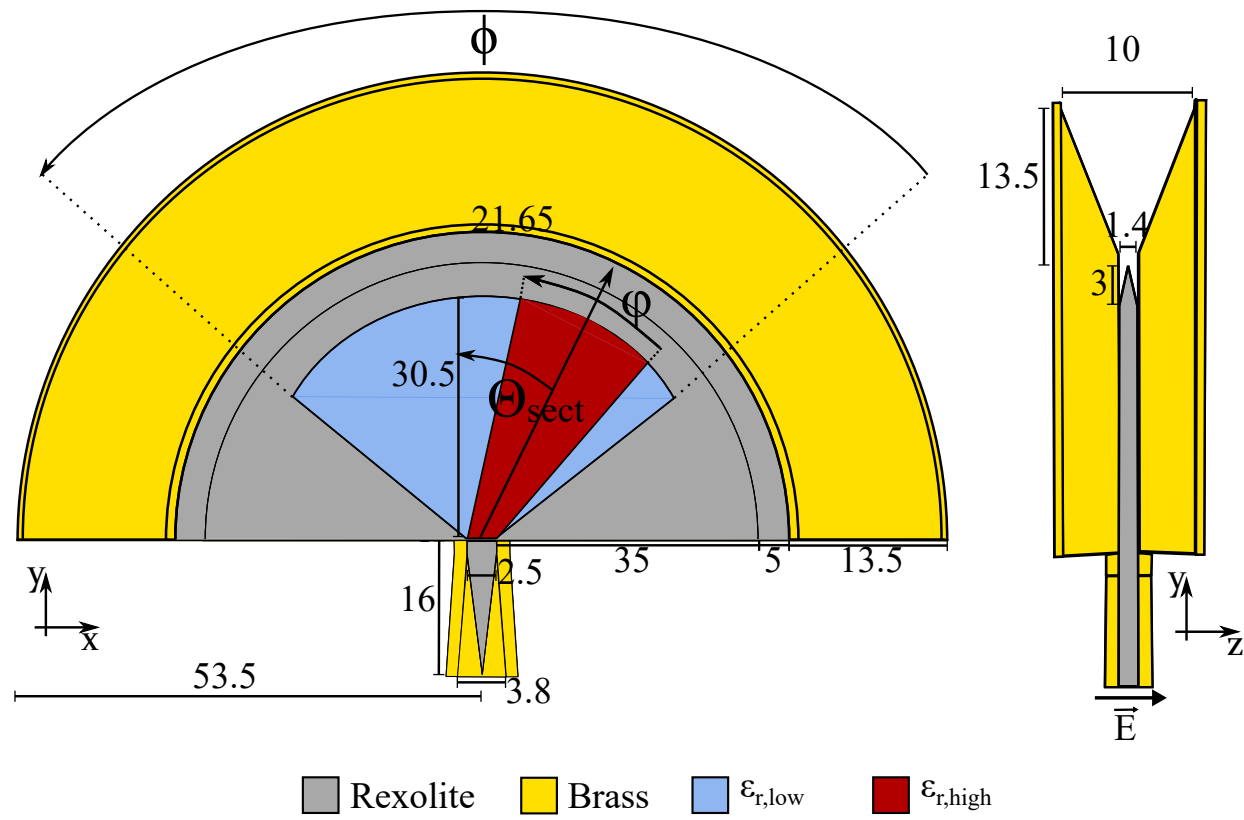

Figure 40. Representation of the lateral wave beam steering antenna in parallel plate topology. Lengths are in $\mathrm{mm}$.

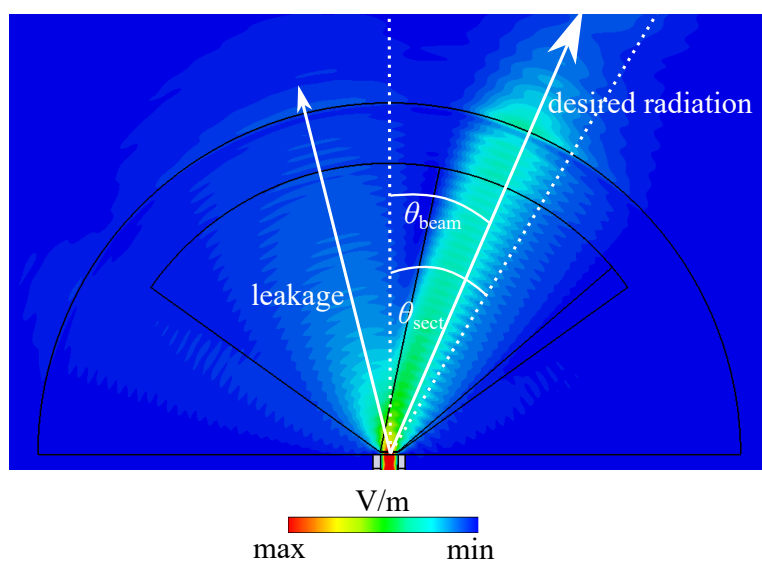

(a)

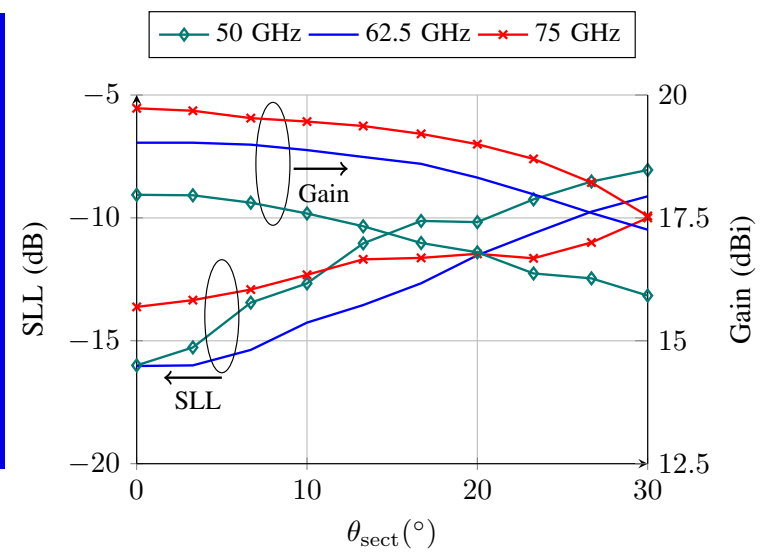

(b)

Figure 41. (a) Full wave simulation of the parallel-plate lateral wave antenna in a steered configuration at $62.5 \mathrm{GHz},(\mathbf{b})$ simulated gain and SLL in dependency of $\theta_{\text {sect }}$ at three selected frequencies in V-band. 


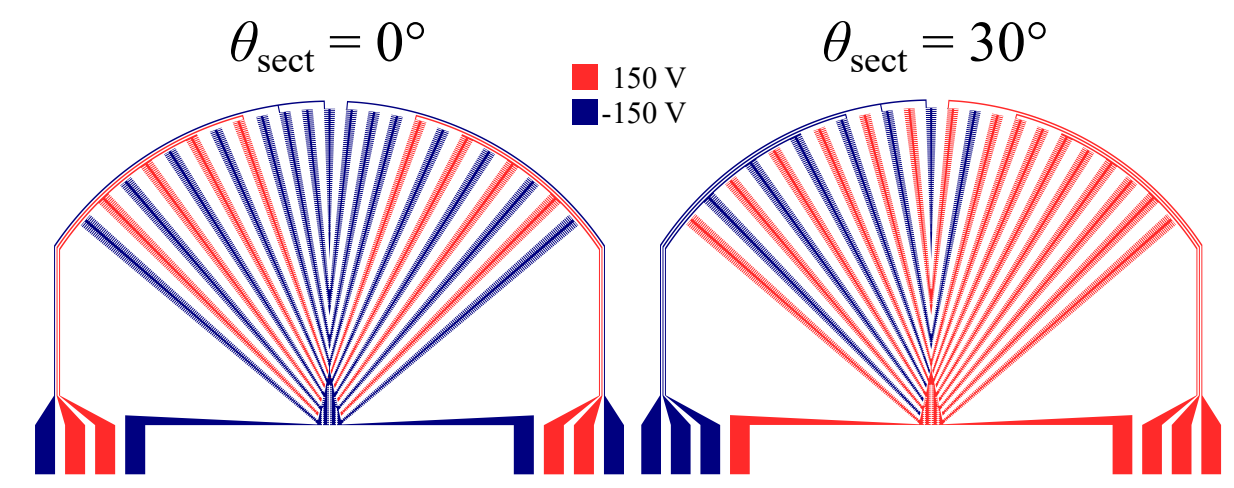

(a) Voltage distribution on the top electrode network
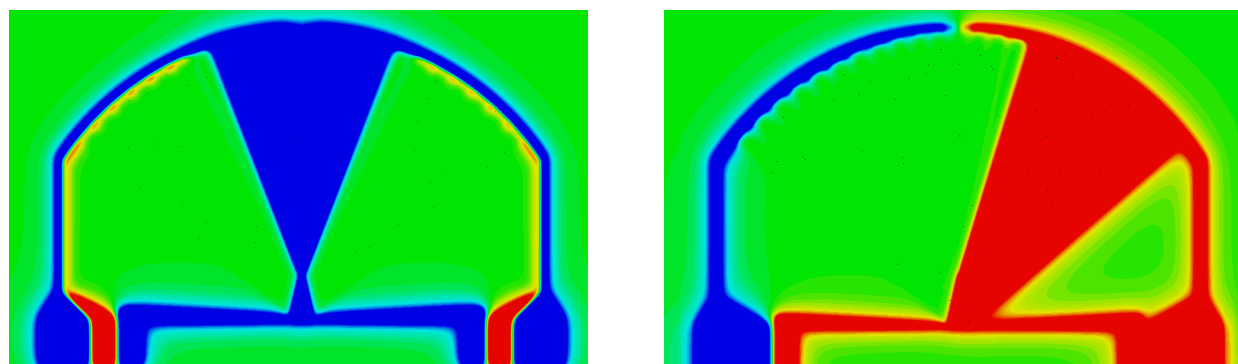

(b) Normal E-field component
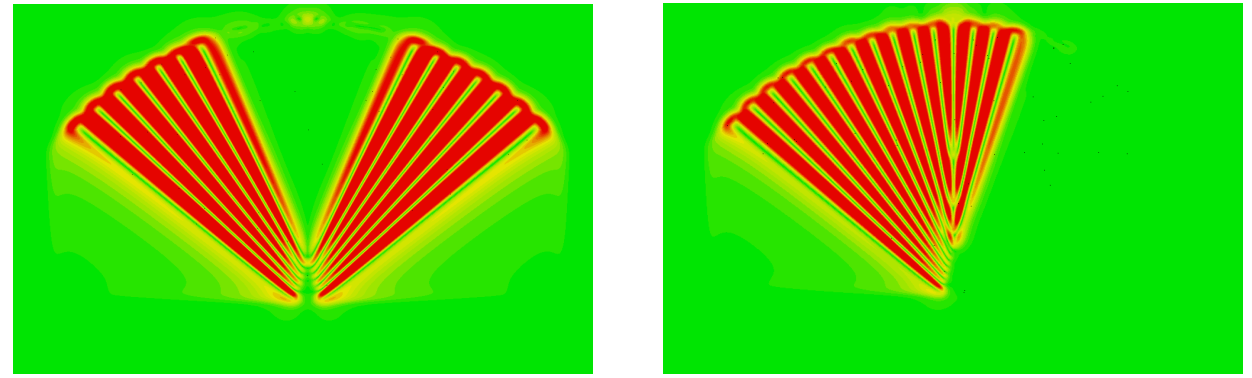

(c) Tangential field components

\begin{tabular}{cc|}
$-100 \mathrm{kV} / \mathrm{m}$ & $100 \mathrm{kV} / \mathrm{m}$ \\
\hline
\end{tabular}

Figure 42. (a) The voltage distribution on the electrode network results in different bias field distributions in the LC filled cavity, leading to components responsible for high permittivity (b) and low permittivity (c). Note the ladder-like stepped impedance structure of the electrodes. [45], reprinted with kind permission of IEEE.

Figure 43a shows the electrode network which is processed on Pyralux substrate and glued to the metallic housing with wafer mounting wax. The second electrode network is glued to the complementary metallic part. After inserting the LC-filled Rexolite body (Figure 43b), and connecting the bias supply to the bias pads, the antenna is fully assembled, see Figure $43 \mathrm{c}$. 


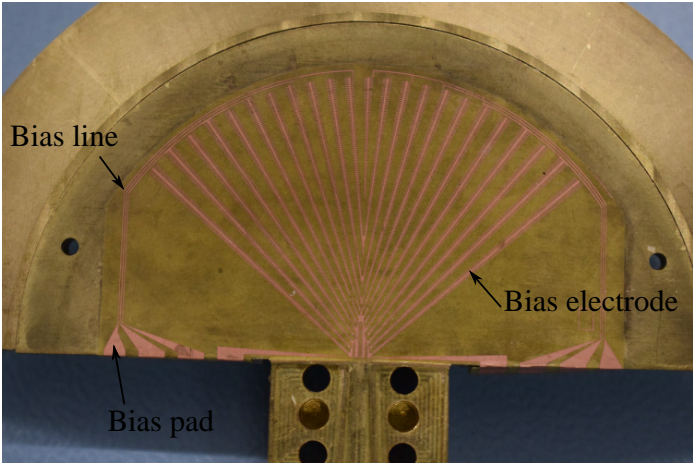

(a)

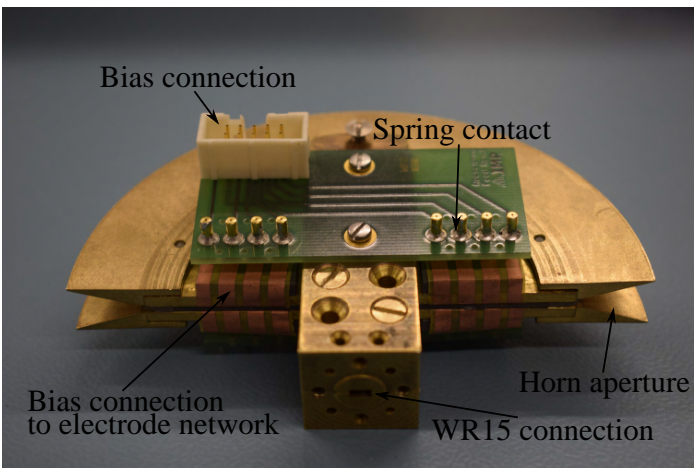

(c)

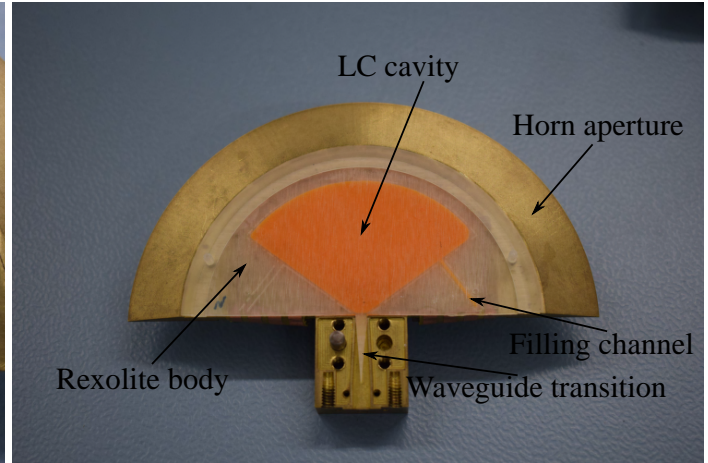

(b)

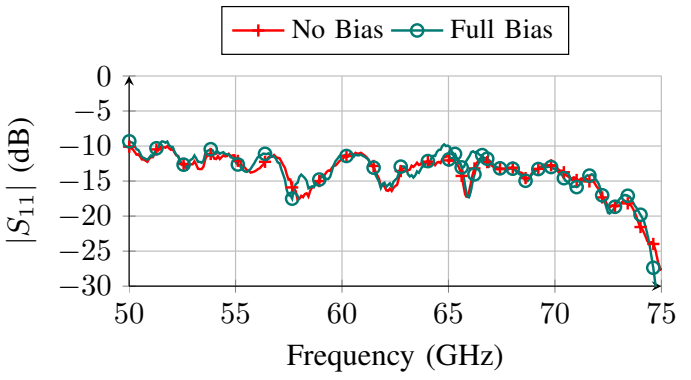

(d)

Figure 43. (a) Top electrode network, (b) inserted Rexolite body with LC, (c) assembled demonstrator, and (d) measured input reflection for different bias states.

The antenna is just matched throughout the whole frequency range in V-band, as Figure 43d indicates. The simulated and measured patterns for $\theta_{\text {beam }}=0^{\circ}$ and $\theta_{\text {beam }}=30^{\circ}$ are depicted in Figure 44. For $\theta_{\text {beam }}=0^{\circ}$, we observe increased HPBW and SLL. The origins of this behavior can be found in a non-perfect transition from high permittivity to low permittivity.

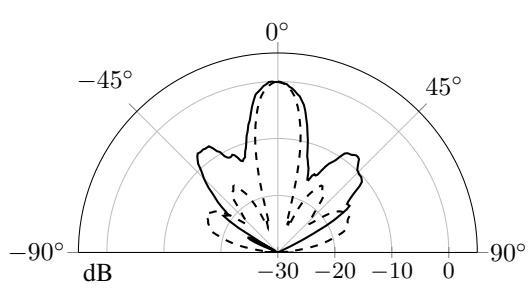

(a) $50 \mathrm{GHz}$

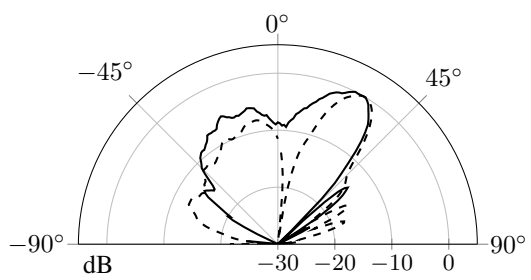

(c) $50 \mathrm{GHz}$

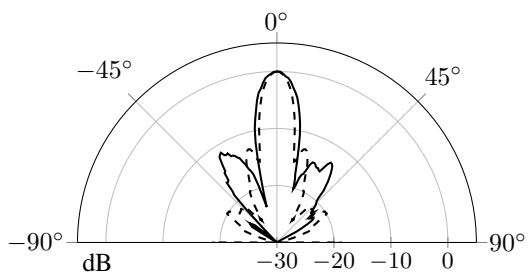

(b) $75 \mathrm{GHz}$

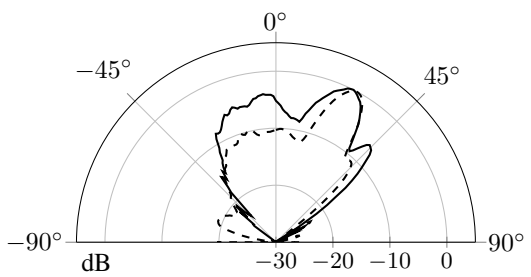

(d) $75 \mathrm{GHz}$

- Measurement - - - Simulation

Figure 44. Pattern of the parallel-plate lens antenna for $(\mathbf{a}, \mathbf{b}) \theta_{\text {beam }}=0^{\circ}$ and for $(\mathbf{c}, \mathbf{d}) \theta_{\text {beam }}=30^{\circ}$. 
A changeover layer from high to low permittivity exists, hence permittivity does not change in an abrupt manner but shows a gradient. This non-perfect boundary of permittivities causes slightly more penetration of the propagating wave to the lower permittivity regions. When the steered configuration is considered, the pattern is well established in the lower frequency regions, but deteriorates with higher frequency. Again, higher leakage from the high permittivity sector are the cause. An overview of simulated and measured gain and SLL is given in Figure 45. Especially the SLL is considerably higher than expected at the steered configuration and gain is in general $3 \mathrm{~dB}$ to $4 \mathrm{~dB}$ lower than simulated. Reduced gain and higher SLL are interlinked properties, since higher leakage from the high permittivity sector results in less power in the main beam and more spurious radiation which leads to higher SLLs. In addition, the stepped impedance structure and the metal casing of the antenna are additional sources of loss.

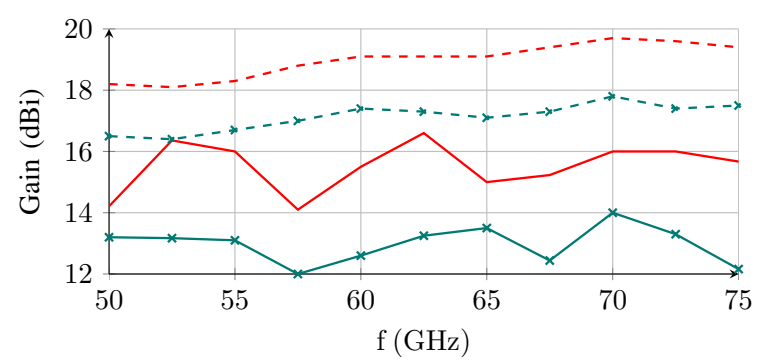

(a)

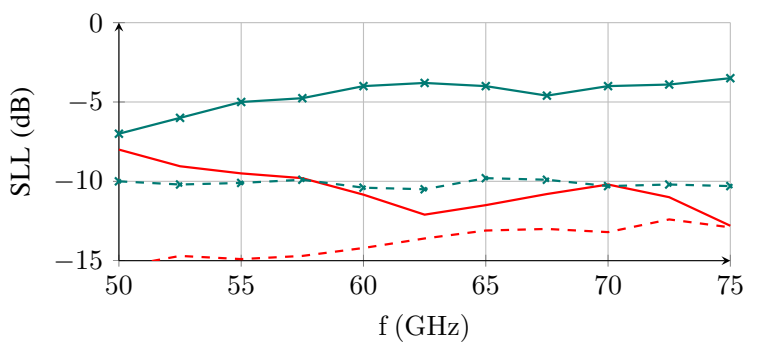

(b)

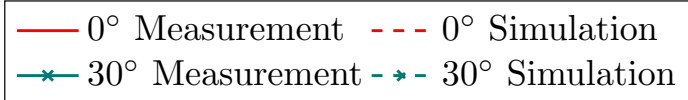

Figure 45. Comparison of simulated and measured (a) gain and (b) SLL behavior for $\theta_{\text {beam }}=0^{\circ}$ and $\theta_{\text {beam }}=30^{\circ}$.

In order to eliminate the influence of metallic boundaries and electrodes, a fully dielectric version of steerable lens antenna has been developed [47]. Its layout with corresponding dimensions is depicted in Figure 46. The semicircular antenna body (C), milled from Rexolite 1422, is fed by a tapered sWL (B) with a Preperm L300 (successor material now known as Preperm PPE300 [48]) core for better matching and reduced radiation loss. As before, the antenna body hosts a cavity of LC. Instead of a horn aperture, a semicircular dielectric lens (D) forms the end of the antenna and ensures increased gain. The transition section (A) is only necessary in order to feed/measure the antenna in the lab with a standard WR-15 hollow waveguide. The diameter of the antenna is $D=80 \mathrm{~mm}$, therefore, it is $25 \%$ smaller than its metallic counterpart. As a consequence, the LC cavity is $10 \mathrm{~mm}$ smaller than in the metallic predecessor. Since [45] shows that high steering angles lead to non-tolerable SLLs, this setup reduces the maximum sector steering angle to $\theta_{\text {sect }} \leq 25^{\circ}$ which results in beam angles of $\theta_{\text {beam }} \approx 20^{\circ}$ for a wide frequency range. With this initial limitation, the LC cavity can be kept smaller and less LC is necessary for the design. However, due to the fully dielectric nature of the antenna, the antenna body has to be $2.5 \mathrm{~mm}$ thick. Otherwise, too much evanescent field components are present outside the dielectric and the LC cavity which results in less influence of change in permittivity at the core of the dielectric. Three different bias states for radiation towards $\theta_{\text {beam }}=20^{\circ}, \theta_{\text {beam }}=0^{\circ}$ and $\theta_{\text {beam }}=-20^{\circ}$ are demonstrated in [47]. The necessary electrode network and the corresponding field components in the different bias configurations are depicted in Figure 47. Note, that simple metallic strips can serve as electrodes, and no stepped-impedance design is necessary. However, the bias voltage has to be increased to $\pm 250 \mathrm{~V}$, since the electrodes have to be moved away from the dielectric body, such that the evanescent field is not disturbed. Nevertheless, the freedom in shape of the electrode allows full customization of the bias network. This ensures that more sophisticated electrode networks are possible and no design restriction is present with the fully dielectric design. In the network shown in Figure 47 this freedom manifests in the gate electrodes, which are used to block or enable wave 
propagation in steered directions. The electrode network is milled on standard FR-4 substrate of $100 \mu \mathrm{m}$ thickness. The antenna is formed by two halves milled from Rexolite 1422 and glued together with UV-glue with the inserted Preperm L300 feed core.

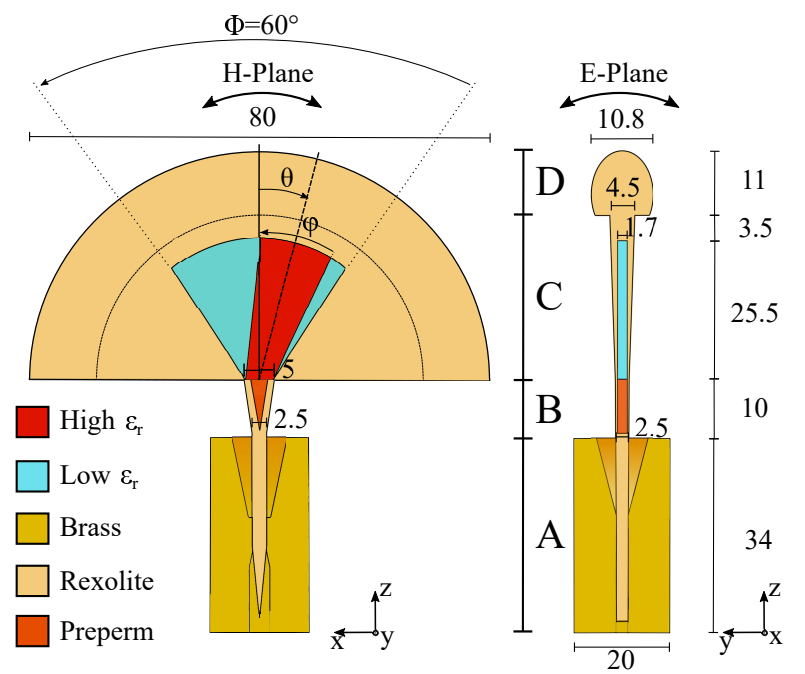

Figure 46. Representation of the fully dielectric lateral wave beam steering antenna [47]. Lengths are in mm. Reprinted with kind permission of IEEE.

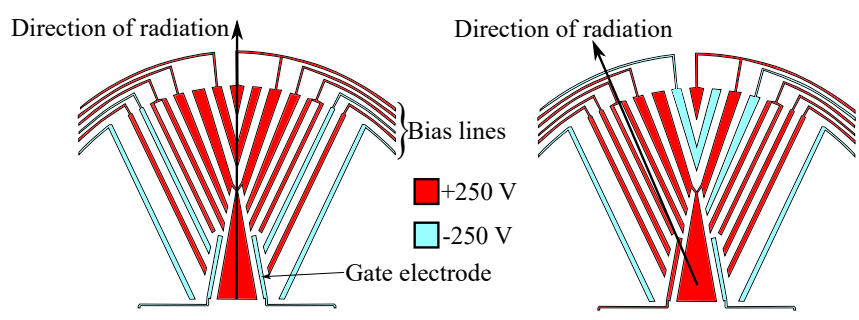

(a) Voltage distribution on the top electrode network
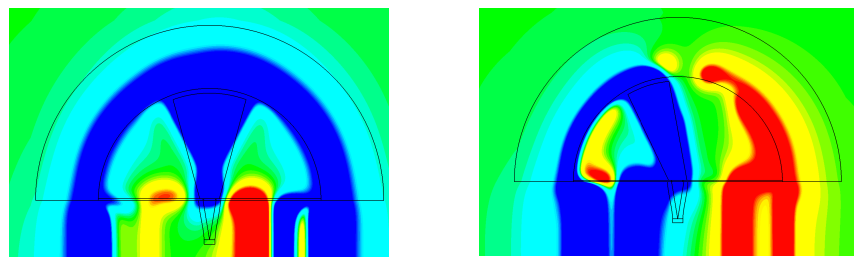

(b) Normal E-field component
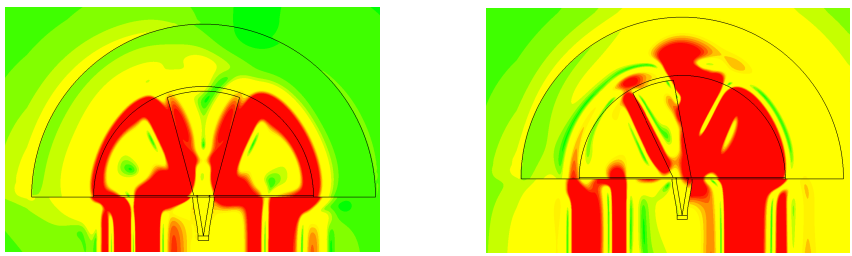

(c) Tangential field components

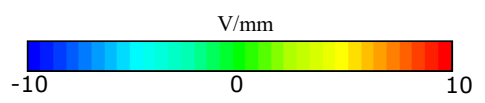

Figure 47. (a) The voltage distribution on the electrode network results in different bias field distributions in the LC filled cavity, leading to components responsible for high permittivity (b) and low permittivity (c). The desired sector of high permittivity is included in $(\mathbf{b}, \mathbf{c})$. [47], reprinted with kind permission of IEEE. 
After insertion of LC GT5-26001 via a filling channel, the channel is sealed with epoxy glue. The electrode network is held in place with the help of the WR-15 transition. In order to ensure parallel alignment of both FR-4 substrates Rohacell 51HF spacers are introduced in between the antenna and the electrode networks. The proposed dielectric antenna has low weight of $16.73 \mathrm{~g}$. When compared to the PPDW antenna [45], which weights $354 \mathrm{~g}$, a 21-fold reduction is achieved, which makes the fully dielectric approach especially useful in weight restricted applications. The fully assembled demonstrator and the antenna itself are depicted in Figure 48.

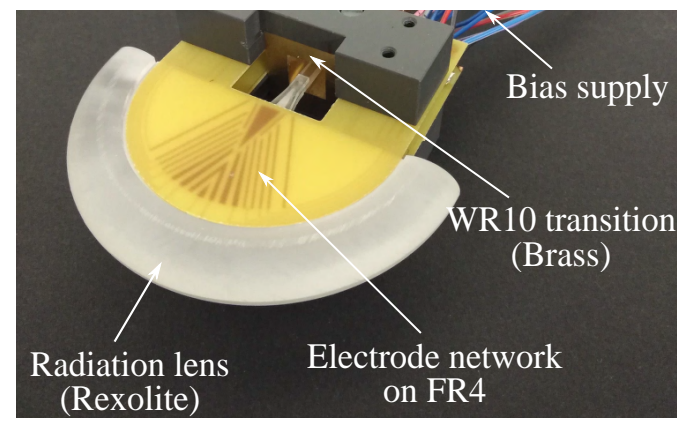

(a)

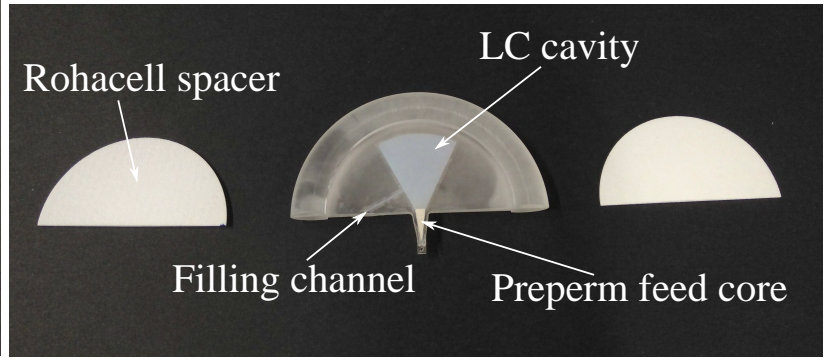

(b)

Figure 48. (a) Fully assembled dielectric lens antenna demonstrator, (b) partly disassembled demonstrator, showing the antenna only. [47], reprinted with kind permission of IEEE.

The antenna is well matched both with no bias and full bias of the whole LC cavity, as Figure 49 shows. The simulated and measured H-plane patterns at highest and lowest frequency of operation, both for $\theta_{\text {beam }}=0^{\circ}$ and $\theta_{\text {beam }}=20^{\circ}$, are compared in Figure 50. Considering the $\theta_{\text {beam }}=0^{\circ}$ confirmation first, we can observe that the pattern is in general wider than predicted. This indicates higher leakage effects than assumed in simulations, where a perfectly abrupt transition from high to low permittivity regions is present. As in [45] a more gradual transition is present in practice since the corresponding field components overlap slightly as Figure 47 indicates. Therefore, the horn aperture is not perfectly represented, and differences between simulation and measurement are the consequence.

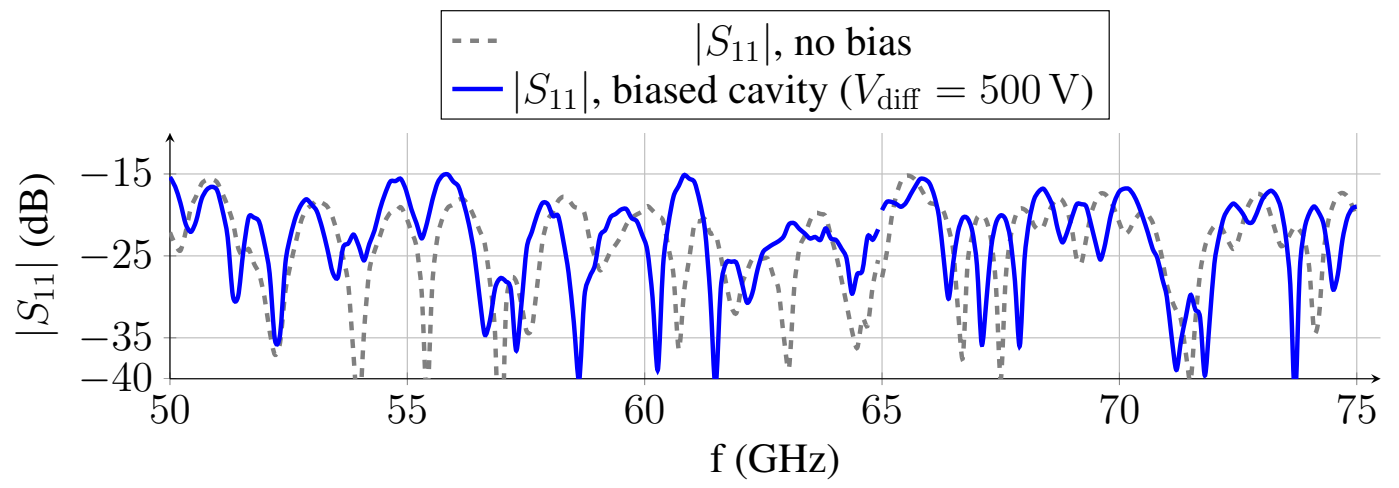

Figure 49. Measured input reflection for an unbiased and fully biased LC cavity.

The pattern obtained when switching to a steered configuration $\left(\theta_{\text {beam }}=20^{\circ}\right)$ match the simulation well, with slight deviations in $\theta_{\text {beam }}$ which is maximally $3^{\circ}$ less than desired ( $17^{\circ}$ instead of $20^{\circ}$ ). This difference can again be explained by the imperfect permittivity boundaries, showing the practical limitation of this steering technique. Figure 51 shows gain and SLL in both $\theta_{\text {beam }}=0^{\circ}$ and $\theta_{\text {beam }}=20^{\circ}$ configurations. For $\theta_{\text {beam }}=0^{\circ}$ gain is $2 \mathrm{~dB}$ to $4 \mathrm{~dB}$ lower than simulated, which is related to the broader pattern measured. The SLL, however, remains very low as predicted. Better agreement in gain is present for $\theta_{\text {beam }}=20^{\circ}$, where only deviations of $0.6 \mathrm{~dB}$ to $2 \mathrm{~dB}$ are present and the SLL matches simulations very well. 


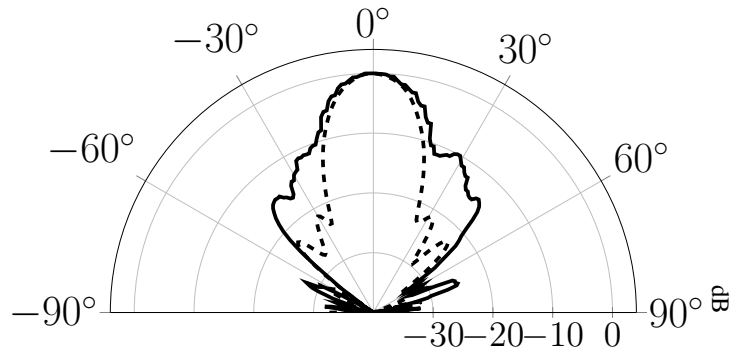

(a) $50 \mathrm{GHz}$

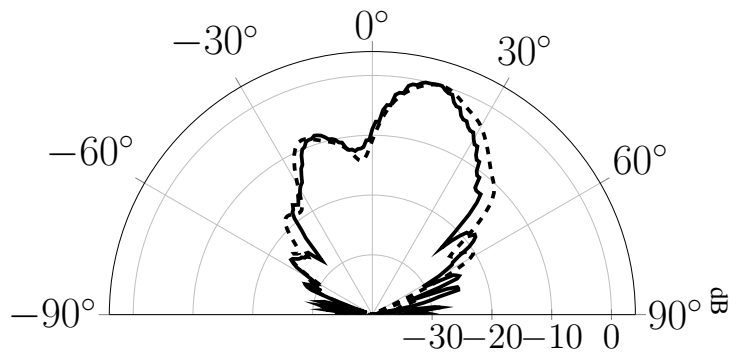

(c) $50 \mathrm{GHz}$

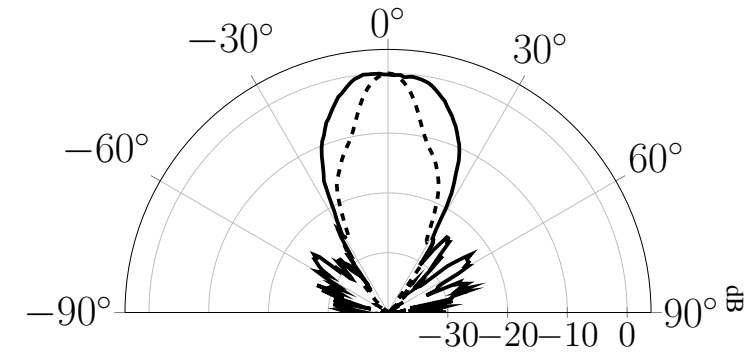

(b) $75 \mathrm{GHz}$

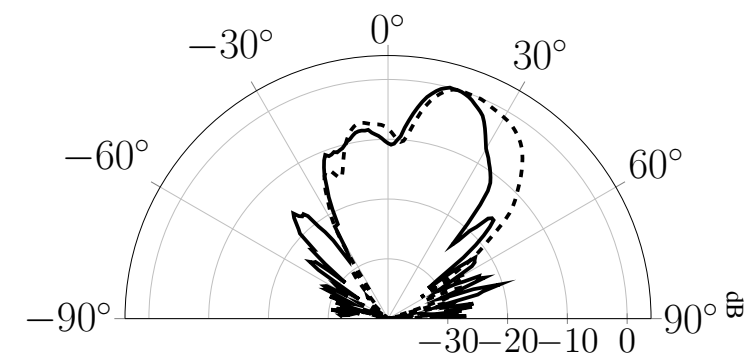

(d) $75 \mathrm{GHz}$

\section{Measurement - - - Simulation}

Figure 50. Pattern of the dielectric lens antenna [47] for $(\mathbf{a}, \mathbf{b}) \theta_{\text {beam }}=0^{\circ}$ and for $(\mathbf{c}, \mathbf{d}) \theta_{\text {beam }}=20^{\circ}$.

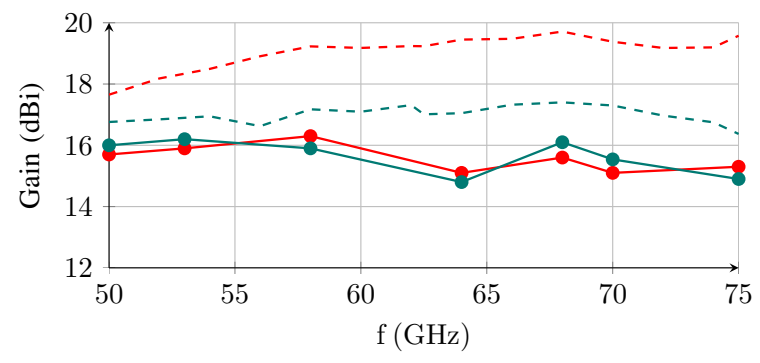

(a)

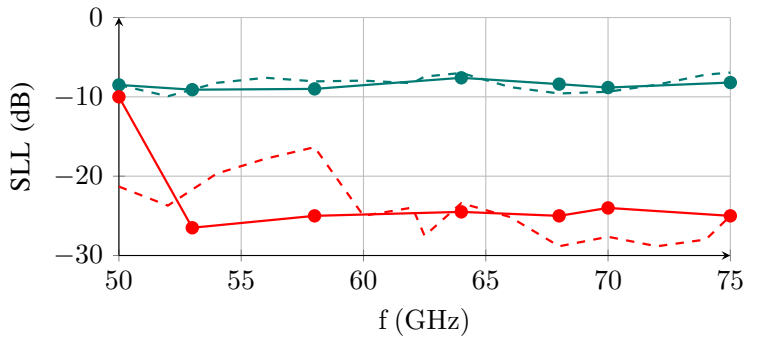

(b)

$$
\begin{aligned}
& -0^{\circ} \text { Measurement }--0^{\circ} \text { Simulation } \\
& --20^{\circ} \text { Measurement }-\bullet 20^{\circ} \text { Simulation }
\end{aligned}
$$

Figure 51. Comparison of simulated and measured (a) gain and (b) SLL behavior for $\theta_{\text {beam }}=0^{\circ}$ and $\theta_{\text {beam }}=20^{\circ}$.

In contrast to [45], especially in the steered configuration, better agreement of simulation and measurement has been achieved in [47]. Nevertheless, the problem of leakage effects are still present in both the metallic and fully dielectric lens antenna. This power leakage can be addressed with a more complex bias electrode network. In case of the fully dielectric antenna, no restrictions in shape of this network are present, such that particular shapes or a pixel grid structure for free sector shape and $\theta_{\text {beam }}$ configurations are possible. The dielectric antenna is very lightweight, but requires higher voltages to be driven. In order to reduce the size of the LC cavity and height of the antenna, a host material of higher permittivity can be used such that the antenna gets smaller and the electrode network can move closer to the antenna body. Closer placed electrodes result in less voltage necessary for biasing the LC, while a reduction of LC cavity height increases response times of the proposed antenna. This is highly desirable, since the high volume of LC and the nature of the used GT5-26001 LC-mixture 
resulted in switching times in the range of a few minutes when switching from $\theta_{\text {beam }}=-20^{\circ}$ to $\theta_{\text {beam }}=20^{\circ}$. Switching time can also be reduced by using a less viscose LC-mixture than GT5-26001. The development towards quickly responding, high performing LC is still ongoing [8], and the newly available GT7-29001 mixture would be a good choice for further investigations and implementations.

\subsection{Filters}

As well as tunable phase shifters and steerable antennas, tunable filters are a key component for reconfigurable RF systems. Therefore, a research field for tunable LC filters emerged in the past years. To obtain tunabilty, the resonators are filled with LC. By controlling the LC's permittivity, the electrical size of the resonator can be adjusted, and therefore, the resonance frequency. A tunable LC waveguide bandpass filter is presented in [49] operating at $20 \mathrm{GHz}$. It has a bandwidth of $1 \%$ and a center frequency tunablity of $450 \mathrm{MHz}$ with magnetic LC biasing. Electric biasing has been shown for single resonators only, since the integration of the electrodes is very challenging. For higher frequencies, this gets even more challenging, due to the decreasing dimensions. Therefore, as well as for LC phase shifters and antennas, dielectric waveguide topologies are considered. Since dielectric waveguides are generally prone to radiation loss at discontinuities, designing filter becomes very challenging. A filter consists of resonators and coupling structures which are discontinuities for the propagating wave, hence it will result in high radiation loss for standard dielectric waveguides as the sWL or SIF fibre. However, the NRD has a nonradiative nature, as mentioned in Section 2.1, since the plate separation is smaller than $\lambda_{0} / 2$. Although the NRD consists of two parallel metal plates, the typical low loss characteristic of dielectric waveguide is preserved, because the plates are parallel to the RF field, otherwise the conduction losses would have been increased. Therefore, the NRD is a promising candidate for designing dielectric waveguide filters, as shown in [25]. Furthermore, it is feasible for a tunable LC filter design. The main advantage of the NRD compared to a waveguide is the open structure, which enables a simple electrode integration even at higher frequencies. In [50] an electrical tunable LC NRD bandpass filter is presented, which operates at $60 \mathrm{GHz}$. With Rexolite as the dielectric material, the NRD is designed with a plate separation of $b=2.3 \mathrm{~mm}$ and a dielectric core with a width of $a=1.9 \mathrm{~mm}$.

There are different ways to design a NRD resonator and coupling structures. One possibility is to use rectangular resonators which are coupled by thin below-cutoff lines [50], as shown in Figure 52. The advantage of this design is that the dielectric can be fabricated out of one part, hence no alignment of the resonators is necessary. In [50], the width of the resonator is set equal to the width $a$ of the NRD and the width of the coupling line is set to $c=250 \mu \mathrm{m}$, due to fabrication limitations. Therefore, only the resonator's $l_{\text {res }}$ and coupling lines' lengths $l_{\text {coup }}$ are changed to adapt the resonance frequency and coupling strengths for the filter design, respectively. To obtain a filter, resonators are coupled with each other and for tunability of the resonance frequency, LC is inserted into the resonators. A graphical representation of a tunable LC NRD 3-pole bandpass filter is shown in Figure 53. A Chebyshev filter function with $1 \%$ factional bandwidth, $0.2 \mathrm{~dB}$ passband ripple level and a center frequency at $60 \mathrm{GHz}$ was aimed for. By using full-wave simulations, the following dimensions of the resonators and coupling lines were obtained in order to meet the desired filter function: $l_{r, 1}=l_{r, 3}=1.37 \mathrm{~mm}$, $l_{r, 2}=1.35 \mathrm{~mm}, l_{c, 1}=l_{c, 4}=2.38 \mathrm{~mm}$, and $l_{c, 2}=l_{c, 3}=4.88 \mathrm{~mm}$. A detailed explanation of the filter design method is given in [50]. The Rexolite wall thickness for the resonators is $200 \mu \mathrm{m}$. The same bias configurations are applied as for the NRD phase shifter presented in Section 2.1.5. However the structure of the electrodes are adapted to the filter's dimensions. 


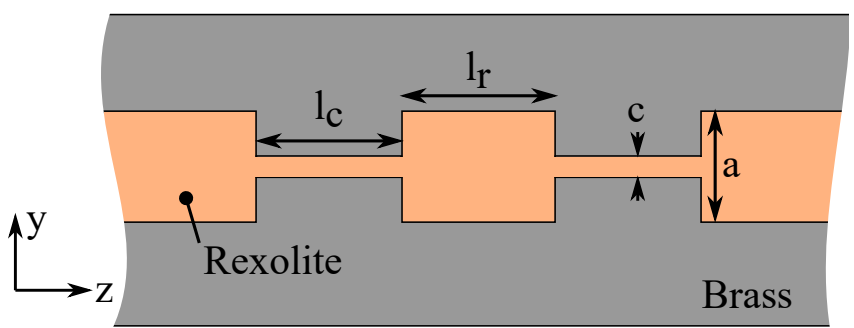

Figure 52. Cross section a NRD resonator which is coupled by below cutoff lines, as presented in [50].

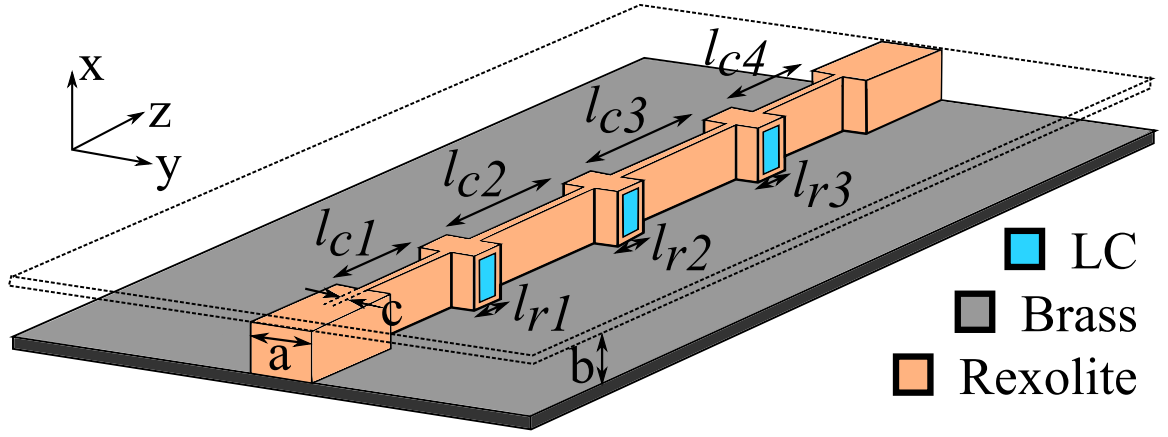

Figure 53. Cross section a 3-pole tunable filter with three NRD resonators coupled by below-cutoff lines, as presented in [50].

The resulting electrode design is depicted in Figure 54. By using slots and stubs, unwanted parasitic mode excitation is avoided. The electrodes are processed on on a $12 \mu \mathrm{m}$-thick Pyralux substrate with a $9 \mu \mathrm{m}$ single copper clad. The substrates are glued onto the parallel metal plates of the NRD. In order to measure the NRD filter and to excite the operating LSM $_{01}$ mode, a transition from WR-15 to the NRD was designed. Afterwards, the NRD is fabricated together with the transitions out of one piece brass in split-block technology. A photograph of the fabricated demonstrator is shown in Figure 55. The measured and simulated S-parameter results with the LC mixture GT3-23001 are shown in Figure 56 for both parallel and orthogonal LC orientation. A bias voltage of $\pm 200 \mathrm{~V}$ was applied and a tuning range of $2.5 \%$ was measured with a insertion loss ranging between $4.9 \mathrm{~dB}$ to $6.2 \mathrm{~dB}$. In order to increase the accuracy of the simulations, the fabrication tolerances are measured and taken as good as possible into account for the simulations. It can be seen, that there is still a small difference between the simulation and measurement results and that the desired $0.2 \mathrm{~dB}$ passband ripple level is vanished, due to the insertion loss. However, the tuning range and insertion loss are in a very good agreement.

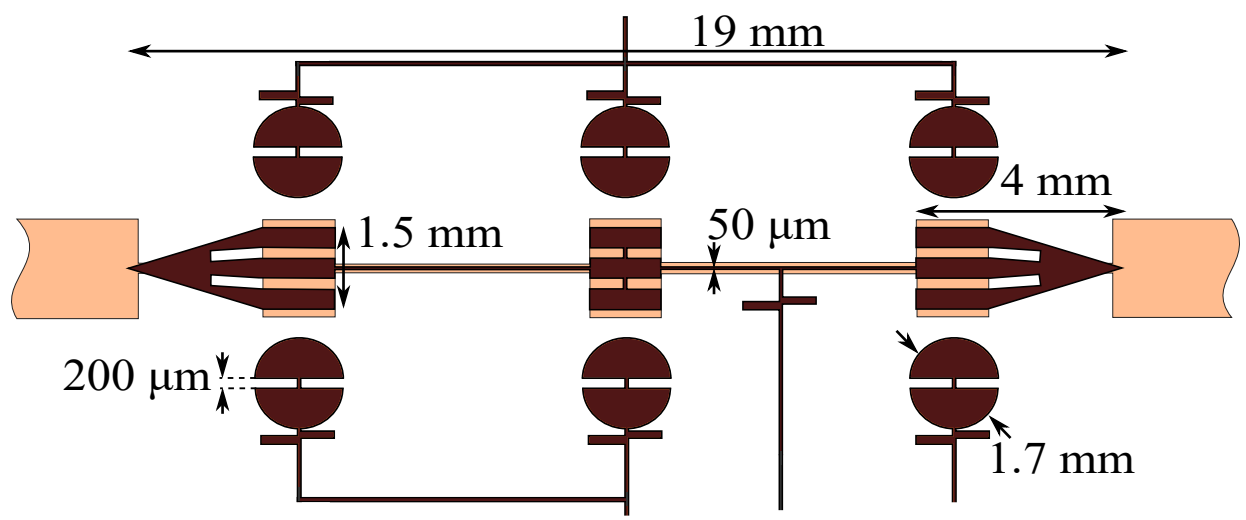

Figure 54. Schematic of the electrode design. 


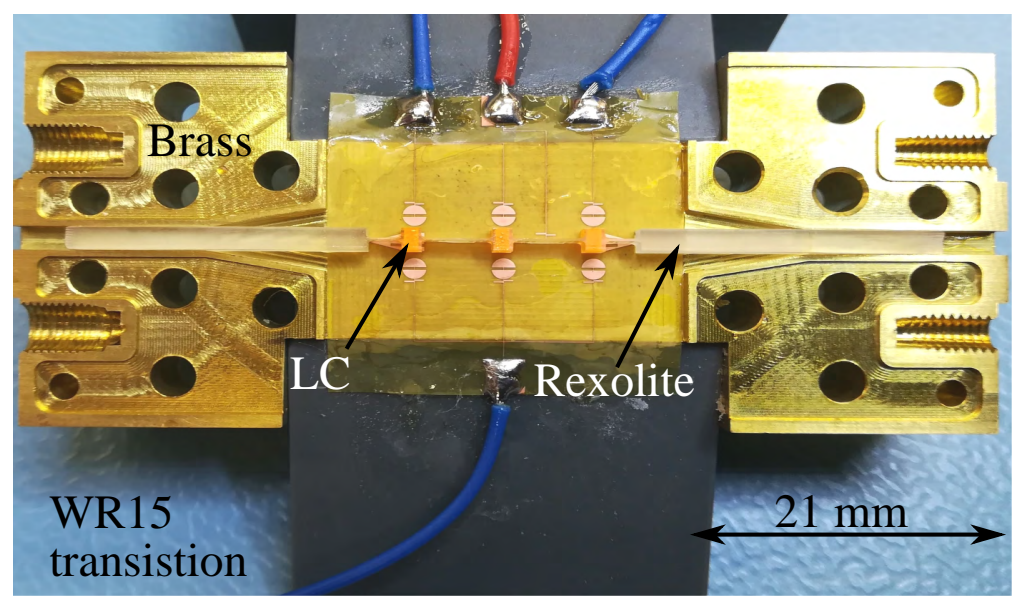

Figure 55. Photograph of the partially assembled tunable NRD bandpass filter.

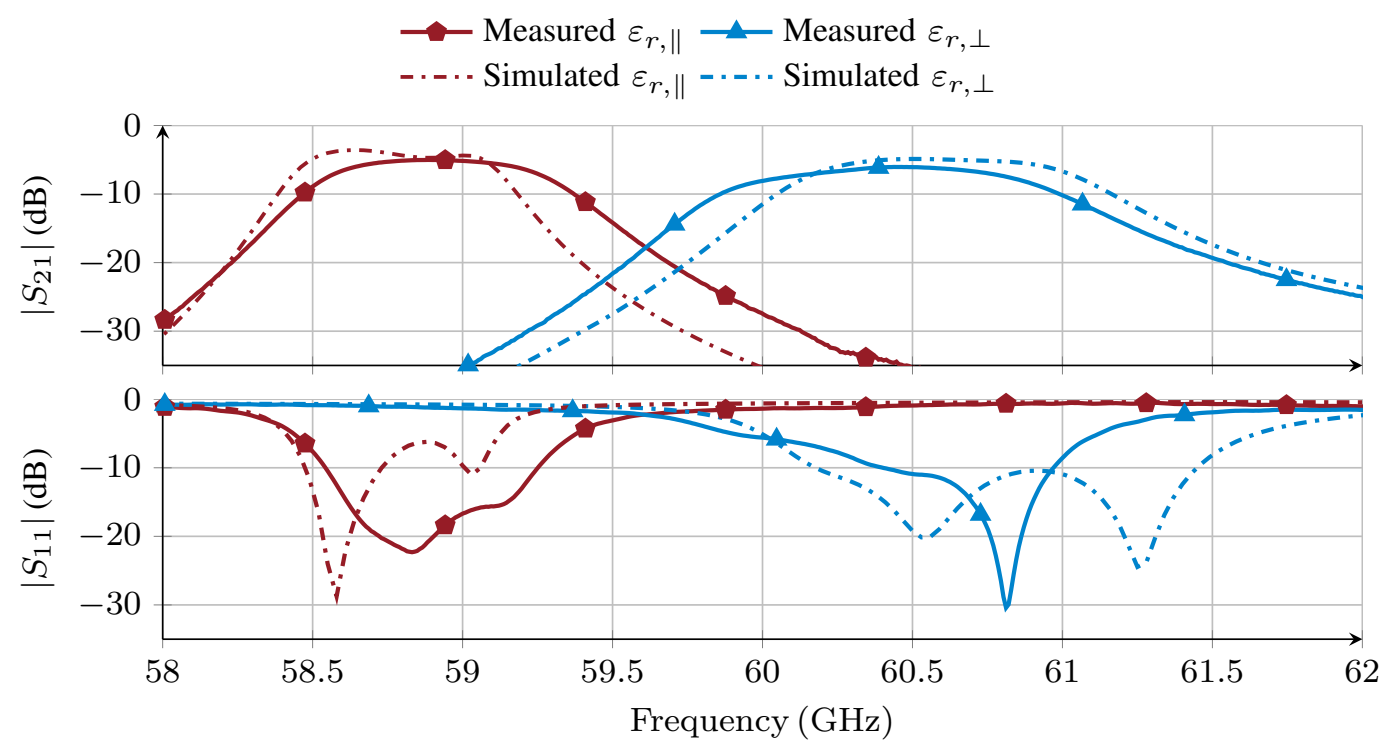

Figure 56. Measured and simulated S-parameters for orthogonal and parallel LC orientation of the presented tunable bandpass filter.

\section{Conclusions}

In this manuscript, the recent developments of tunable smart components based on liquid crystal (LC) in the millimeter wave regime are reviewed. With increasing frequencies, the advantage of dielectric topologies over classic metallic waveguide topologies is getting evident. This trend is confirmed by various LC application examples throughout the paper, as bias electrode integration proves to be comparably simple, inexpensive manufacturing is possible and the resulting components are lightweight. In addition, non-conventional fabrication processes, such as $3 \mathrm{D}$ printing, can be used for mass-manufacturing. In [51] a first demonstrator of a 3D printed Fresnel lens with LC integration is presented, which enables control of the focusing properties of the lens. Different dielectric waveguide topologies suitable for LC-integration are presented, each with its own advantages and disadvantages. The subwavelength fibre (sWL) prove to be the best performing phase shifter regarding the figure-of-merit (FoM), however, its response time and comparably high bias voltage remain an issue to overcome. In order to do so, the dielectric image line (DIL) or the nonradiative dielectric waveguide (NRD) can be employed at the cost of higher loss. Therefore, the dielectric waveguide topology always has to be chosen accordingly to the desired application.

The main application in this review of LC phase shifters is in phased array antennas. The two demonstrators in this manuscript rely on the sWL topology, since it is simple to realize and shows 
high performance. The first demonstrator is a hybrid approach, with both metallic and dielectric components, which cause degradation of the antenna pattern. Therefore, a fully dielectric approach is preferable, since no change of topology is present. Multimode interference (MMI), a phenomenon known from optics, provides a possibility to realize compact, fully dielectric power dividers. Lateral wave beam steering antennas based on LC offer an alternative to the common phased array approach. By changing the antenna aperture beam steering and beam forming can be achieved. A fully dielectric approach shows better defined patterns at the cost of switching speed.

The realization of filters using dielectric waveguides is generally challenging, since they are prone to radiation losses at discontinuities. However, the NRD, as its name indicates, is radiation free even at bends and discontinuities. This enables the design of resonators and filters, without suffering from radiation losses. By applying LC into the resonators of a filter, tunable filters can be realized.

Overall, it has been shown that dielectric topologies are well suited for LC-based applications in the $\mathrm{mmW}$ regime. However, in order to unlock the full potential of dielectric LC-based $\mathrm{mmW}$ components, the material properties of LC has to be fully characterized at these frequencies for more accurate simulative predictions. A widespread overview of LC mixture characterization at lower frequencies is already present [7], but has to be extended to higher frequencies. Furthermore, other unknowns in this frequency range, such as loss of applied glue and dielectric materials have to be eliminated. With the increasing utilization of LC in microwave applications, the the field of microwave liquid crystal is a dynamic field of research with ongoing advance [8].

Author Contributions: Conceptualization: E.P., H.T., H.M.; Investigation: E.P., H.T., R.R.; Writing: E.P., H.T.; Review \& Editing, E.P., H.T., R.R., M.N., D.W., P.S., R.J., H.M.; Supervision, H.M. and R.J.; Funding Acquisition, H.M. and R.J. All authors have read and agreed to the published version of the manuscript.

Funding: This research was partially funded by DFG grants JA921/29, JA921/68-1, JA921/72-1, MA6281/4-1.

Acknowledgments: The authors gratefully acknowledge Merck KGaA for supplying Liquid Crystal mixtures. We acknowledge support by the German Research Foundation and the Open Access Publishing Fund of Technische Universität Darmstadt.

Conflicts of Interest: The authors declare no conflict of interest.

\section{Abbreviations}

The following abbreviations are used in this manuscript:

$\begin{array}{ll}\text { 5G } & \text { System for Mobile Communication of the 5th generation } \\ \text { DIL } & \text { Dielectric image line } \\ \text { FoM } & \text { Figure of Merit } \\ \text { HPBW } & \text { Half-power beam width } \\ \text { IL } & \text { Insertion loss }\left(\mathrm{IL}=-\left|S_{21}\right|\right) \\ \text { LC } & \text { Liquid Crystal } \\ \text { MIMO } & \text { Multiple-input-multiple-output } \\ \text { MMI } & \text { Multimode interference } \\ \text { mmW } & \text { Millimeter wave } \\ \text { NRD } & \text { Nonradiative dielectric waveguide } \\ \text { PPDW } & \text { Parallel plate dielectric waveguide } \\ \text { RF } & \text { Radio frequency } \\ \text { SIF } & \text { Stepped-index fibre } \\ \text { sWL } & \text { Subwavelength } \\ \text { UV } & \text { Ultraviolet } \\ \text { WLAN } & \text { Wireless local area network }\end{array}$

\section{References}

1. Cudak, M.; Ghosh, A.; Kovarik, T.; Ratasuk, R.; Thomas, T.A.; Vook, F.; Moorut, P. Moving Towards Mmwave-Based Beyond-4G (B-4G) Technology. IEEE Veh. Technol. Conf. (VTC) 2013. [CrossRef] 
2. Shafi, M.; Molisch, A.F.; Smith, P.J.; Haustein, T.; Zhu, P.; De Silva, P.; Tufvesson, F.; Benjebbour, A.; Wunder, G. 5G: A Tutorial Overview of Standards, Trials, Challenges, Deployment, and Practice. IEEE J. Sel. Areas Commun. 2017, 35, 1201-1221. [CrossRef]

3. Ben-Dor, E.; Rappaport, T.S.; Qiao, Y.; Lauffenburger, S.J. Millimeter-Wave $60 \mathrm{GHz}$ Outdoor and Vehicle AOA Propagation Measurements Using a Broadband Channel Sounder. In Proceedings of the IEEE Global Telecommunications Conference (GLOBECOM), Houston, TX, USA, 5-9 December 2011. [CrossRef]

4. Kyro, M.; Ranvier, S.; Kolmonen, V.; Haneda, K.; Vainikainen, P. Long range wideband channel measurements at $81-86$ GHz frequency range. In Proceedings of the European Conference on Antennas and Propagation (EuCAP), Barcelona, Spain, 12-16 April 2010; pp. 1-5.

5. Hoefle, M.; Koeberle, M.; Penirschke, A.; Jakoby, R. Improved millimeter wave Vivaldi antenna array element with high performance liquid crystals. In Proceedings of the 2011 International Conference on Infrared, Millimeter, and Terahertz Waves, Houston, TX, USA, 2-7 October 2011. [CrossRef]

6. Weil, C.; Luessem, G.; Jakoby, R. Tunable inverted-microstrip phase shifter device using nematic liquid crystals. In Proceedings of the 2002 IEEE MTT-S International Microwave Symposium Digest (Cat. No.02CH37278), Seattle, WA, USA, 2-7 June 2002. [CrossRef]

7. Zografopoulos, D.C.; Ferraro, A.; Beccherelli, R. Liquid-Crystal High-Frequency Microwave Technology: Materials and Characterization. Adv. Mater. Technol. 2018. [CrossRef]

8. Fritzsch, C.; Wittek, M. Recent developments in liquid crystals for microwave applications. In Proceedings of the IEEE International Symposium on Antennas and Propagation \& USNC/URSI National Radio Science Meeting, San Diego, CA, USA, 9-14 July 2017. [CrossRef]

9. Polat, E.; Reese, R.; Tesmer, H.; Schmidt, S.; Spaeth, M.; Nickel, M.; Schuster, C.; Jakoby, R.; Maune, H. Characterization of Liquid Crystals Using a Temperature-Controlled $60 \mathrm{GHz}$ Resonator. In Proceedings of the IEEE MTT-S International Microwave Workshop Series on Advanced Materials and Processes for RF and THz Applications (IMWS-AMP), Bochum, Germany, 16-18 July 2019; pp. 19-21. [CrossRef]

10. Weickhmann, C.; Jakoby, R.; Constable, E.; Lewis, R.A. Time-domain spectroscopy of novel nematic liquid crystals in the terahertz range. In Proceedings of the 2013 38th International Conference on Infrared, Millimeter, and Terahertz Waves (IRMMW-THz), Mainz, Germany, 1-6 September 2013; pp. 1-2.

11. Maune, H.; Jost, M.; Reese, R.; Polat, E.; Nickel, M.; Jakoby, R. Microwave Liquid Crystal Technology. Crystals 2018, 8, 355. [CrossRef]

12. Natarajan, A.; Reynolds, S.K.; Tsai, M.; Nicolson, S.T.; Zhan, J.C.; Kam, D.G.; Liu, D.; Huang, Y.O.; Valdes-Garcia, A.; Floyd, B.A. A Fully-Integrated 16-Element Phased-Array Receiver in SiGe BiCMOS for 60-GHz Communications. IEEE J. Solid State Circuits 2011, 46, 1059-1075. [CrossRef]

13. Henoch, B.T.; Tamm, P. A 360/spl deg/Reflection-Type Diode Phase Modulator (Correspondence). IEEE Trans. Microw. Theory Tech. 1971, 19, 103-105. [CrossRef]

14. Zheng, Y.; Saavedra, C.E. An Ultra-Compact CMOS Variable Phase Shifter for 2.4-GHz ISM Applications. IEEE Trans. Microw. Theory Tech. 2008, 56, 1349-1354. [CrossRef]

15. Jost, M. Liquid Crystal Mixed Beam-Switching and Beam-Steering Network in Hybrid Metallic and Dielectric Waveguide Technology. Ph.D. Thesis, TU Darmstadt, Darmstadt, Germany, 2018.

16. Weickhmann, C.; Nathrath, N.; Gehring, R.; Gaebler, A.; Jost, M.; Jakoby, R. Recent measurements of compact electronically tunable liquid crystal phase shifter in rectangular waveguide topology. Electron. Lett. 2013, 49, 1345-1347. [CrossRef]

17. Gaebler, A.; Goelden, F.; Manabe, A.; Goebel, M.; Mueller, S.; Jakoby, R. Investigation of high performance transmission line phase shifters based on liquid crystal. In Proceedings of the European Microwave Conference (EuMC), Rome, Italy, 29 September-1 October 2009; pp. 594-597. [CrossRef]

18. Jost, M.; Weickhmann, C.; Strunck, S.; Gaebler, A.; Fritzsch, C.; Karabey, O.H.; Jakoby, R. Liquid crystal based low-loss phase shifter for W-band frequencies. Electron. Lett. 2013, 49, 1460-1462. [CrossRef]

19. Jost, M.; Weickhmann, C.; Strunck, S.; Gaebler, A.; Hu, W.; Franke, T.; Prasetiadi, A.E.; Karabey, O.H.; Jakoby, R. Electrically biased W-band phase shifter based on liquid crystal. In Proceedings of the International Conference on Infrared, Millimeter, and Terahertz waves (IRMMW-THz), Tucson, AZ, USA, 14-19 September 2014; pp. 1-2. [CrossRef]

20. Reese, R.; Polat, E.; Tesmer, H.; Strobl, J.; Schuster, C.; Nickel, M.; Granja, A.B.; Jakoby, R.; Maune, H. Liquid Crystal Based Dielectric Waveguide Phase Shifters for Phased Arrays at W-Band. IEEE Access 2019, 7, 127032-127041. [CrossRef] 
21. Friedsam, G.L.; Biebl, E.M. Precision free-space measurements of complex permittivity of polymers in the W-band. In Proceedings of the IEEE MTT-S International Microwave Symposium (IMS), Denver, CO, USA, 8-13 June 1997. [CrossRef]

22. Reese, R.; Jost, M.; Maune, H.; Jakoby, R. Design of a continuously tunable W-band phase shifter in dielectric waveguide topology. In Proceedings of the IEEE MTT-S International Microwave Symposium (IMS), Honololu, HI, USA, 4-9 June 2017; pp. 180-183. [CrossRef]

23. Tesmer, H.; Reese, R.; Polat, E.; Jakoby, R.; Maune, H. Dielectric Image Line Liquid Crystal Phase Shifter at W-Band. In Proceedings of the German Microwave Conference (GeMiC), Montreal, QC, Canada, 9-11 March 2020; pp. 156-159.

24. Reese, R.; Polat, E.; Jost, M.; Nickel, M.; Jakoby, R.; Maune, H. Liquid crystal based phase shifter in a parallel-plate dielectric waveguide topology at V-band. In Proceedings of the European Microwave Integrated Circuits Conference (EuMIC), Nuremberg, Germany, 8-10 October 2017; pp. 353-356. [CrossRef]

25. Yoneyama, T.; Kuroki, F.; Nishida, S. Design of Nonradiative Dielectric Waveguide Filters (Correction). IEEE Trans. Microw. Theory Tech. 1985, 33, 741-741. [CrossRef]

26. Polat, E.; Reese, R.; Jost, M.; Nickel, M.; Schuster, C.; Jakoby, R.; Maune, H. Liquid Crystal Phase Shifter Based on Nonradiative Dielectric Waveguide Topology at W-Band. In Proceedings of the IEEE MTT-S International Microwave Symposium (IMS), Boston, MA, USA, 2-7 June 2019; pp. 184-187. [CrossRef]

27. Sanadgol, B.; Holzwarth, S.; Kassner, J. $30 \mathrm{GHz}$ liquid crystal phased array. In Proceedings of the Loughborough Antennas Propagation Conference, Loughborough, UK, 16-17 November 2009; pp. 589-592. [CrossRef]

28. Karabey, O.H.; Gaebler, A.; Strunck, S.; Jakoby, R. A 2-D Electronically Steered Phased-Array Antenna With $2 \times 2$ Elements in LC Display Technology. IEEE Trans. Microw. Theory Tech. 2012, 60, 1297-1306. [CrossRef]

29. Heunisch, A.; Schulz, B.; Rabe, T.; Strunck, S.; Follmann, R.; Manabe, A. LTCC Antenna Array with Integrated Liquid Crystal Phase Shifter for Satellite Communication. Addit. Conf. 2012, 2012, 97-102. [CrossRef]

30. Hoehn, A.; Hager, P.B.; Harder, J.T. Design characterization of an electronic steerable Ka-band antenna using liquid crystal phase shifters. In Proceedings of the IEEE Aerospace Conference, Big Sky, MT, USA, 2-9 March 2013. [CrossRef]

31. Weickhmann, C.; Nathrath, N.; Gehring, R.; Gaebler, A.; Jost, M.; Jakoby, R. A light-weight tunable liquid crystal phase shifter for an efficient phased array antenna. In Proceedings of the European Microwave Conference (EuMC), Nuremberg, Germany, 6-10 October 2013; pp. 428-431. [CrossRef]

32. Maune, H.; Weickhmann, C.; Jost, M.; Reese, R.; Nickel, M.; Fritzsch, C.; Jakoby, R. Liquid crystal technology for reconfigurable satcom applications. In Proceedings of the Topical Workshop on Internet of Space (TWIOS), Phoenix, AZ, USA, 15-18 January 2017. [CrossRef]

33. Richter, J.; Muller, M.; Schmidt, L.P. Measurement of phase centers of rectangular dielectric rod antennas. In Proceedings of the IEEE Antennas and Propagation Society International Symposium, Monterey, CA, USA, 20-25 June 2004. [CrossRef]

34. Richter, J.; Schmidt, L. Dielectric Rod Antennas as Optimized Feed Elements for Focal Plane Arrays. In Proceedings of the IEEE Antennas and Propagation Society International Symposium, Washington, DC, USA, 3-8 July 2005. [CrossRef]

35. Polat, E.; Reese, R.; Tesmer, H.; Nickel, M.; Jakoby, R.; Maune, H. Fully Dielectric Phased Array for Beamsteering Using Liquid Crystal Technology at W-Band. In Proceedings of the European Conference on Antennas and Propagation (EuCAP), Copenhagen, Denmark, 15-20 March 2020.

36. Soldano, L.B.; Pennings, E.C.M. Optical multi-mode interference devices based on self-imaging: Principles and applications. J. Light. Technol. 1995, 13, 615-627. [CrossRef]

37. Bachmann, M.; Besse, P.A.; Melchior, H. General self-imaging properties in N x N multimode interference couplers including phase relations. Appl. Opt. 1994, 33, 3905-3911. [CrossRef]

38. Reese, R.; Jost, M.; Nickel, M.; Polat, E.; Jakoby, R.; Maune, H. A Fully Dielectric Lightweight Antenna Array Using a Multimode Interference Power Divider at W-Band. IEEE Antennas Wirel. Propag. Lett. 2017, 16, 3236-3239. [CrossRef]

39. Reese, R.; Jost, M.; Polat, E.; Nickel, M.; Jakoby, R.; Maune, H. Beam Steering Capabilities of a Fully Dielectric Antenna Array. In Proceedings of the IEEE International Symposium on Antennas and Propagation \& USNC/URSI National Radio Science Meeting, Boston, MA, USA, 8-13 July 2018. [CrossRef] 
40. Reese, R.; Tesmer, H.; Jost, M.; Polat, E.; Nickel, M.; Jakoby, R.; Maune, H. A Compact Two-dimensional Power Divider for a Dielectric Rod Antenna Array Based on Multimode Interference. J. Infrared Millim. Terahertz Waves 2018, 39, 1185-1202. [CrossRef]

41. Reese, R.; Tesmer, H.; Polat, E.; Jost, M.; Nickel, M.; Jakoby, R.; Maune, H. Fully Dielectric Rod Antenna Arrays with High Permittivity Materials. In Proceedings of the German Microwave Conference (GeMiC), Stuttgart, Germany, 25-27 March 2019. [CrossRef]

42. Tesmer, H.; Reese, R.; Polat, E.; Nickel, M.; Jakoby, R.; Maune, H. Fully Dielectric Rod Antenna Arrays with Integrated Power Divider. Frequenz 2019, 73, 367-377. [CrossRef]

43. Evonik Industries AG. Technical Information Rohacell HF. Available online: https://www.rohacell. com/sites/lists/RE/DocumentsHP/ROHACELL\%20HF\%20Product\%20Information.pdf (accessed on 25 February 2020).

44. Tokan, F.; Tokan, N.T.; Neto, A.; Cavallo, D. The Lateral Wave Antenna. IEEE Trans. Antennas Propag. 2014, 62, 2909-2916. [CrossRef]

45. Reese, R.; Jost, M.; Polat, E.; Tesmer, H.; Strobl, J.; Schuster, C.; Nickel, M.; Jakoby, R.; Maune, H. A Millimeter-Wave Beam-Steering Lens Antenna With Reconfigurable Aperture Using Liquid Crystal. IEEE Trans. Antennas Propag. 2019, 67, 5313-5324. [CrossRef]

46. Singh, A.K.; Chaudhari, R.; Jha, B.; Jha, R.K. Radiation pattern of E-plane sectoral solid dielectric horn antennas. Int. J. Electron. 1992, 73, 203-213. [CrossRef]

47. Tesmer, H.; Reese, R.; Polat, E.; Nickel, M.; Jakoby, R.; Maune, H. Liquid-Crystal-Based Fully Dielectric Lateral Wave Beam-Steering Antenna. IEEE Antennas Wirel. Propag. Lett. 2019, 18, 2577-2581. [CrossRef]

48. Premix Oy. Premix Standard Grades. Available online: https://www.preperm.com/products/rawmaterials/\#preperm-standard-grades (accessed on 25 February 2020).

49. Franke, T.; Gaebler, A.; Prasetiadi, A.E.; Jakoby, R. Tunable Ka-band waveguide resonators and a small band band-pass filter based on liquid crystals. In Proceedings of the European Microwave Conference (EuMC), Rome, Italy, 6-9 October 2014; pp. 339-342. [CrossRef]

50. Polat, E.; Reese, R.; Jost, M.; Schuster, C.; Nickel, M.; Jakoby, R.; Maune, H. Tunable Liquid Crystal Filter in Nonradiative Dielectric Waveguide Technology at 60 GHz. IEEE Microw. Wirel. Compon. Lett. 2019, 29, 44-46. [CrossRef]

51. Nose, T.; Ito, R.; Honma, M. Potential of Liquid-Crystal Materials for Millimeter-Wave Application. Appl. Sci. 2018, 8, 2544. [CrossRef] 